Pontifícia $U_{\text {niversidade }}$ Católica $_{\text {do Rio de Janeiro }}$

Mateus Bastos Neiva

\title{
Representação numérica do comportamento de cascas axissimétricas obtido da combinação de soluções analíticas fundamentais
}

\section{Projeto de Graduação}

Projeto de Graduação apresentado ao Departamento de Engenharia Mecânica da PUC-Rio.

Orientador: Carlos Alberto de Almeida 


\section{Agradecimentos}

A Solange Pereira Bastos, matriarca da família, grande exemplo de vida que tive durante vinte anos ao proporcionar ensinamentos valiosos de generosidade, carinho e amor. Como professora de formação, tinha uma sabedoria incomparável, anos dedicando-se em garantir o melhor ensino possível a seus netos. Responsável pelo meu ingresso no Colégio de São Bento e pelo meu desejo em cursar Engenharia.

Aos meus pais, Cláudia Maria Pereira Bastos Neiva e José Antonio Lisbôa Neiva, pelo amor e carinho que sempre tiveram comigo e com meu irmão. Além disso, referências de inspiração e exemplos de profissionalismo e competência. À minha mãe pela dedicação, amor, preocupação e atenção e ao meu pai pelo grande coração, amor e bondade. Agradeço por acreditarem e investirem no meu estudo.

Ao meu irmão, afilhado e melhor amigo, Gustavo Bastos Neiva, pelo companherismo e amizade que há vinte anos temos. Cooperação tanto nos momentos difíceis que passamos e quanto nos de alegria. Agradeço pela sua paciência em revisar meus textos, em especial o presente trabalho.

Uma singela homenagem a André Luiz Bastos Garcia, meu primo, pelo bom coração, alegria cativante, exemplo de vida e carinho que tocou por completo o coração da minha família, deixando-nos profundamente desamparados com sua ida prematura.

Ao meu orientador, Carlos Alberto de Almeida, por toda dedicação e empenho. Muito além de inteligência pessoal, garantiu o aprendizado de tantas gerações, marcado por sua didática e elevado nível de ensino. Agradeço pela paciência no desenvolvimento deste trabalho e pelo quanto cresci em conhecimento.

A Harry Gustavo Saaveda Espinoza e a João Carlos Virgolino Soares pelo excelente trabalho feito, que serviu como base para alavancar o meu estudo e fazer o meu projeto de fim de curso.

Aos meus amigos e colegas que estiveram comigo ao longo de cinco anos cursando Engenharia. Mais que uma cooperação nas horas difíceis de estudos garantiram alegria, descontração e muitas conversas. Em especial Henrique Reis Santiago, grande amigo de longa data, João Pedro Cunha, Marcelo Durães, Brunna Accardo, Anna Letícia Alegria, Antonio Galvão, Lucas Sagrilo, Caio Filippo, Beatriz Viñas, Roberto Carvalho, Gabriela Wegmann, Julia Moreira, Murilo Oliveira, Francisco Mendes, e muitos mais que marcaram minha vida universitária. 


\section{Resumo}

\section{Representação numérica do comportamento de cascas axis- simétricas obtido da combinação de soluções analíticas fun- damentais}

Neste projeto desenvolveu-se um modelo de elementos finitos enriquecidos para simulação estrutural de vasos de pressão sujeitos à rotação axial e à pressão (interna ou externa). A solução analítica do deslocamento correspondente a casos representativos do problema é obtida sem que funções genéricas de interpolação sejam empregadas, como é clássico em programas de elementos finitos. Desta forma, a função interpoladora dos deslocamentos resulta da combinação de soluções analíticas previamente conhecidas. Esta é obtida da combinação das soluções analíticas para os deslocamentos de cilindros com deformação axial nula, para cilindros com rotação e para deslocamentos em esferas. Cada elemento foi formulado com 4 nós, dispostos axialmente na superfície média da casca. A cada nó estão associados sete graus de liberdade equivalentes aos coeficientes das funções de deslocamento nas direções axial e radial. O desenvolvimento da formulação de elementos finitos enriquecidos apresenta a vantagem de, ao se empregar a função interpoladora que melhor quantifica os resultados nodais, não ser necessário um número elevado de elementos para se observar a convergência do método. Também, para garantir continuidade dos deslocamentos entre os elementos, certificando-se que além dos mesmos deslocamentos também a primeira derivada destes é contínua, emprega-se o método de penalidades. Por fim, consideram-se na avaliação do modelo numérico com esta formulação os resultados da variação das tensões ao longo da espessura do vaso de pressão.

\section{Palavras-chave}

Elementos Finitos Enriquecidos; Cascas Axissimétricas; Vaso de Pressão. 


\section{Abstract}

\section{Numerical representation of the behavior of axisymmetric shells obtained by a combination of fundamentals analyt- ical solutions}

In this work, an enriched finite element model is developed for structural simulation on vessels of pressure subjected to axial rotation and under internal and external pressure loads. An analytical solution for displacement, in some cases, is recovered without applying interpolating functions, as usually done on commercial finite element software. So, interpolating functions for displacement comes from combining previously known analytical solutions such: the solution for cylinders under zero axial strain, for cylinders under rotation and for the sphere. Each element was formulated using for four nodes, arranged axially on middle surface of the cylindrical shell. To each nodal point it is associated seven degrees-of-freedom, referring to the coefficients of the displacement function, on axial and radial directions. The development of an enriched finite elements has the advantage of choosing a function that best quantify the nodal point results, with no need of a high number of elements to obtain numerical convergence of the method. In addition, to ensure displacement continuity between two adjacent elements, certifying that beyond the same displacement the first derivative is continuous, the penalty method is applied. Finally, the variation of stress through the thickness of the vessel of pressure is considered, on the numerical model.

\section{Keywords}

Finite Element Method; Axisymmetric shells; Vessel of pressure 


\section{Sumário}

$1 \quad$ Soluções Analíticas $\quad 11$

\begin{tabular}{lll}
\hline 1.1 & Solução de Lamé & 11
\end{tabular}

$\begin{array}{lll}1.2 & \text { Solução para Esferas } & 17\end{array}$

\begin{tabular}{lll}
\hline 1.3 & Disco Girante & 21
\end{tabular}

$\begin{array}{lll}1.4 & \text { Resumo das soluções analíticas } & 28\end{array}$

2 Modelo de cascas $\quad 30$

$2.1 \quad$ Introdução à teoria de cascas finas 30

$\begin{array}{lll}2.2 & \text { Teoria de cascas } & 39\end{array}$

3 Desenvolvimento do Método dos Elementos Finitos 46

$\begin{array}{lll}3.1 & \text { Introdução } & 46\end{array}$

3.2 Funções de deslocamento 47

3.3 Condições de contorno naturais 48

$\begin{array}{lll}3.4 & \text { Funções lagrangeanas } & 49\end{array}$

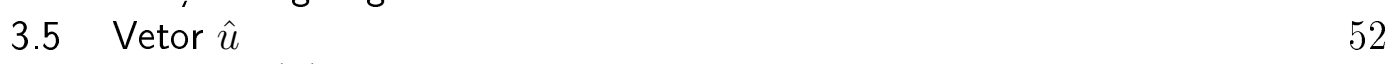

$\begin{array}{lll}3.6 & \text { Matriz } H^{(m)} & 52\end{array}$

3.7 Matriz B 54

$\begin{array}{lll}3.8 & \text { Matriz C } & 57\end{array}$

3.9 Matriz de Rigidez K 58

3.10 Vetor Carregamento 59

3.11 Condição de Continuidade 63

3.12 Condição de Fixação 67

\begin{tabular}{lll}
\hline 4 & Programa & 70
\end{tabular}

$\begin{array}{lll}5 & \text { Resultados Numéricos } & 71\end{array}$

$\begin{array}{lll}5.1 & \text { Cilindro sujeito à pressão } & 71\end{array}$

\begin{tabular}{lll}
\hline 5.2 & Cilindro sujeito à rotação & 77
\end{tabular}

$\begin{array}{lll}6 & \text { Conclusão } & 84\end{array}$

$\begin{array}{lll}7 & \text { Apêndice } & 85\end{array}$

$\begin{array}{lll}7.1 & \text { Apêndice - Fórmula 1 } & 85\end{array}$

7.2 Apêndice - Condições de Contorno Naturais 87

$\begin{array}{lll}7.3 & \text { Apêndice - Matriz C } & 90\end{array}$

$\begin{array}{lll}7.4 & \text { Apêndice - Matriz B } & 92\end{array}$ 


\section{Lista de figuras}

$1 \quad$ Vaso de pressão cilíndrico 9

2 Vaso de pressão esférico 9

\begin{tabular}{lll}
\hline 3 & Turbina hidrelétrica & 10
\end{tabular}

1.1 Elemento infinitesimal 11

1.2 Seção reta de um cilindro sob pressão interna e externa 14

$\begin{array}{lll}1.3 & \text { Elemento infinitesimal tridimensional } & 17\end{array}$

$\begin{array}{lll}1.4 & \text { Elemento infinitesimal plano } & 17\end{array}$

1.5 Elemento infinitesimal do disco girante 21

$\begin{array}{lll}2.1 & \text { Superfície } & 30\end{array}$

$\begin{array}{lll}2.2 & \text { Curvaturas principais } & 36\end{array}$

2.3 Casca com deformação 40

2.4 Casca 44

3.1 Disposição dos nós no elemento 50

5.1 Tensão radial nó 1 72

5.2 Tensão circunferencial nó 1 72

5.3 Deformação radial nó 1

5.4 Deformação circunferencial nó 1

5.5 Deslocamento radial nó 1

5.6 Tensão radial nó 1 74

5.7 Tensão circunferencial nó 1 74

5.8 Deslocamento radial nó 1

$\begin{array}{lll}5.9 & \text { Tensão radial nó } 1 & 75\end{array}$

5.10 Tensão circunferencial nó 1 75

5.11 Deformação radial nó 1 76

5.12 Deformação circunferencial nó 1

5.13 Tensão radial nó 1 76

5.14 Tensão circunferencial nó 1 76

5.15 Deformação radial nó 1

5.16 Deformação circunferencial nó 1 77

5.17 Tensão radial ao longo da espessura no nó 1

5.18 Tensão circunferencial ao longo da espessura no nó 1

5.19 Deslocamento radial nó 1

5.20 Tensão radial ao longo da espessura no nó 1

5.21 Tensão circunferencial ao longo da espessura no nó 1

5.22 Deslocamento radial nó 1

5.23 Distribuição de tensão para um disco girante 82

5.24 Tensão de Mises nó 1 


\section{Notação}

$-r \rightarrow$ Raio

- $\sigma_{r} \rightarrow$ Tensão radial

- $\sigma_{c} \rightarrow$ Tensão circunferencial

- $\sigma_{y p} \rightarrow$ Resistência ao escoamento

- $\epsilon_{r} \rightarrow$ Deformação radial

- $\epsilon_{c} \rightarrow$ Deformação circunferencial

$-\nu \rightarrow$ Coeficiente de poisson

- $E \rightarrow$ Modulo de elasticidade

$-\omega \rightarrow$ Rotação

- $\rho \rightarrow$ Massa específica

$-p_{\text {int }} \rightarrow$ Pressão interna

$-p_{\text {ext }} \rightarrow$ Pressão externa

$-R_{\text {int }} \rightarrow$ Raio interna

$-R_{\text {ext }} \rightarrow$ Raio externa

- $W \rightarrow$ Deslocamento radial

- $U_{1} \rightarrow$ Deslocamento longitudinal

- $U_{2} \rightarrow$ Deslocamento circunferencial

$-d s \rightarrow$ Comprimento infinitesimal entre os pontos $P$ e $P^{\prime}$ na superfície média

$-\hat{t} \rightarrow$ Vetor tangente

- $\hat{X} \rightarrow$ Vetor posição espacial do ponto genérico $P$

$-\hat{K} \rightarrow$ Vetor curvatura

- $\xi, \theta, \zeta \rightarrow$ Coordenadas locais

- $\Pi \rightarrow$ Funcional da energia potencial total

- $\epsilon_{\xi \xi} \rightarrow$ Componente da deformação na direção $\xi$

- $\epsilon_{\theta \theta} \rightarrow$ Componente da deformação na direção $\theta$

- $\epsilon_{\zeta \zeta} \rightarrow$ Componente da deformação na direção $\zeta$

- $\gamma_{\xi \theta} \rightarrow$ Componente da deformação na direção $\xi \theta$

- $\gamma_{\xi \zeta} \rightarrow$ Componente da deformação na direção $\xi \zeta$

- $\gamma_{\zeta \theta} \rightarrow$ Componente da deformação na direção $\zeta \theta$ 
- $\sigma_{\xi \xi} \rightarrow$ Componente da tensão na direção $\xi$

- $\sigma_{\theta \theta} \rightarrow$ Componente da tensão na direção $\theta$

- $\sigma_{\zeta \zeta} \rightarrow$ Componente da tensão na direção $\zeta$

- $\sigma_{\xi \theta} \rightarrow$ Componente da tensão na direção $\xi \theta$

- $\sigma_{\xi \zeta} \rightarrow$ Componente da tensão na direção $\xi \zeta$

- $\sigma_{\zeta \theta} \rightarrow$ Componente da tensão na direção $\zeta \theta$

- $\alpha \rightarrow$ Parâmetro de penalidade

- $\underline{B} \rightarrow$ Matriz de deformação - deslocamento

- $\underline{C} \rightarrow$ Matriz de constitutiva associada ao elemento axissimétrico

- $E \rightarrow$ Módulo de elasticidade

- $\underline{H} \rightarrow$ Matriz função de interpolação

- $\underline{J} \rightarrow$ Matriz jacobiana de transformação

$-\underline{K} \rightarrow$ Matriz de rigidez 


\section{Introdução}

Vasos de pressão são importantes equipamentos no armazenamento de fluidos ou de resíduos. O seu adequado do dimensionamento é uma importante tarefa na prática da engenharia, requerendo o emprego de conceitos dos mais simples aos mais complexos, como da avaliação da estimativa de vida em junções de geometria diversa. Uma decorrência direta do emprego de vasos de pressão está no armazenamento, esfericamente de gases, que, geralmente estão submetidos a elevadas pressões - e algumas vezes também a elevadas temperaturas - proporcionando uma mistura composta de líquido e gás. Na prática utilizam-se vasos de pressão em duas geometrias básicas: cilíndrica ou esférica. A primeira geralmente utilizada no armazenamento de líquidos enquanto que a segunda é utilizada no armazenamento de gases, este sempre a mais altas pressões do que o primeiro. Esta característica resulta do fato de que no vaso cilíndrico as tensões no casco são maiores (o dobro) de que as tensões de avaliação no casco do vaso esférico.

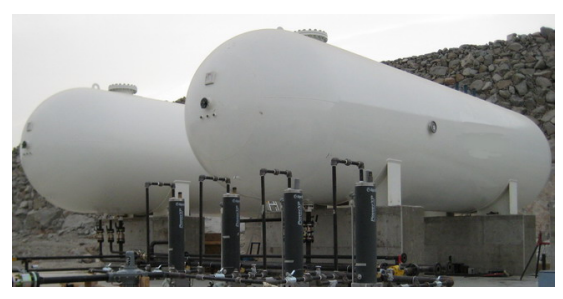

Figura 1: Vaso de pressão cilíndrico

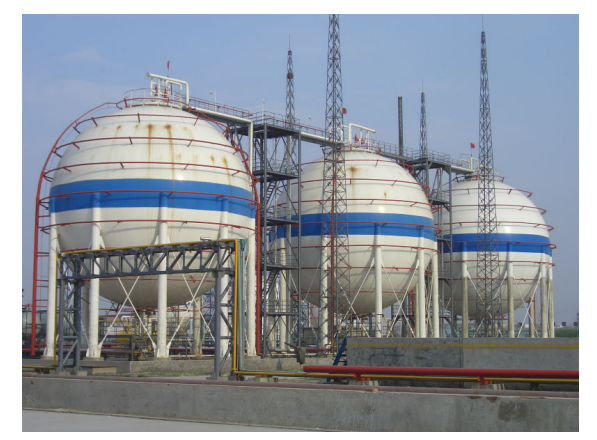

Figura 2: Vaso de pressão esférico

Nas figuras 1 e 1 estão ilustradas aplicações desde dois tipos de geometria de vasos de pressão. A teoria desenvolvida neste trabalho pode, contudo, ser utilizada na avaliação das tensões em vasos ou estruturas para diferentes finalidade. Assim, dutos utilizados para o transporte de óleo e gás são es- 
truturas que apresentam o comportamento semelhante ao dos vasos de pressão.

Neste trabalho são apresentados os aspectos teóricos e de implementação de um modelo estrutural de geometrias de cascas axissimétricas submetidas às pressões internas e externas e também às forças de corpo rígido associadas à inércia da rotação. Uma aplicação importante associada a este estudo é a representação da interferência de montagem entre o eixo e o corpo de uma turbina conforme está mostrado na figura 3 . Esta montagem é realizada, geralmente, por diferença em dilatação térmica.

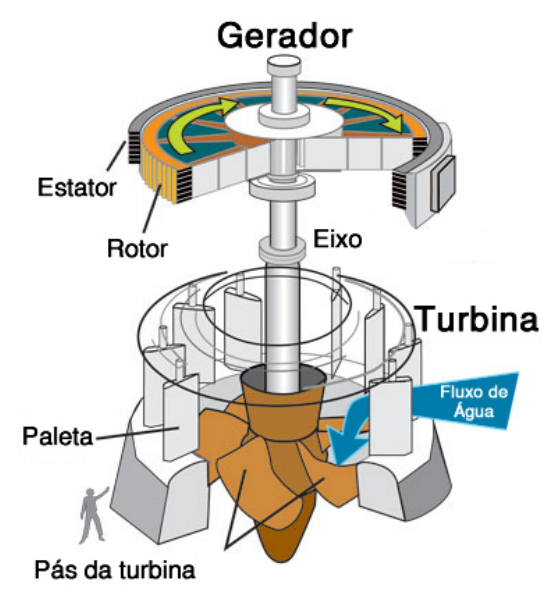

Figura 3: Turbina hidrelétrica

Para a modelagem do problema utilizou-se o método dos elementos finitos, capaz de resolver as distribuições de tensão em corpos de geometria e carregamentos complexos. Na formulação do método adotou-se um elemento de quatro nós dispostos na direção axial utilizando funções interpoladoras de deslocamento de terceiro grau na direção axial, enquanto na direção radial a função fruto da combinação de soluções analíticas conhecidas.

Neste trabalho o primeiro capítulo estão apresentadas as soluções analíticas que serão combinadas para formular a função de deslocamento radial. No segundo capítulo é estudada a teoria das cascas para obter-se as equações de deformações. E, no terceiro capítulo formula-se o método dos elementos finitos enriquecidos utilizando as equações obtidas nos capítulos um e dois. No quarto capítulo é brevemente apresentado o programa elaborado para realizar os cálculos numéricos enquanto no quinto capítulo apresentam-se os resultados obtidos com o modelo numérico formulado. No sexto capítulo apresentam-se as considerações finais e conclusões do trabalho. 


\section{Soluções Analíticas}

No desenvolvimento teórico de modelos de elementos finitos é tradição empregar-se funções interpoladoras polinomiais de lagrange. Neste estudo, no entanto, o deslocamento radial do modelo emprega a combinação de três deslocamentos teóricos: a solução de Lamé, a solução para esferas e a solução para cilindros com rotação. Desta forma, a seguir, desenvolvem-se as soluções analíticas para estes três modelos.

\section{1}

\section{Solução de Lamé}

Desenvolvida originalmente pelo engenheiro fracês Lamé, sua solução trata o problema de vasos de pressões de cilindros de comprimento longo com restrição de deslocamento axial.

\section{Desenvolvimento:}
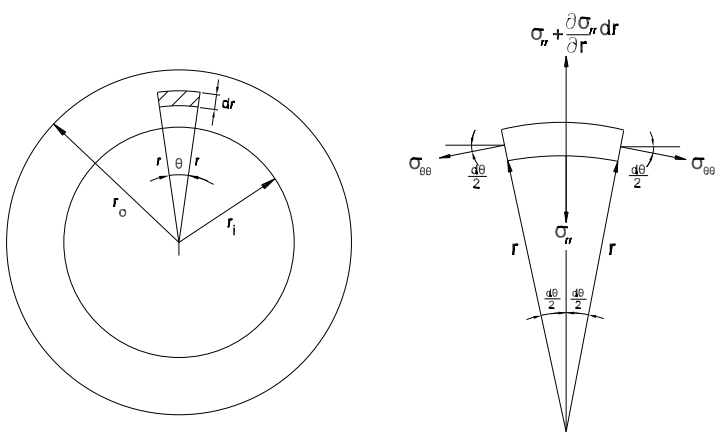

Figura 1.1: Elemento infinitesimal

Aplicando o equilíbrio de forças, como mostra a figura acima 1.1:

$$
\sum F_{\hat{r}}=0
$$

obtem-se

$$
\sigma_{r} d A_{1}-\left(\sigma_{r}+\frac{d \sigma}{d r} d r\right) d A_{2}+2 \sigma_{c} d A_{3} \sin \frac{d \phi}{2}=0
$$

Onde:

$$
d A_{1}=r \cdot d \phi \cdot z \quad d A_{2}=(r+d r) \cdot d \phi \cdot z \quad d A_{3}=d r \cdot z
$$


Portanto, substituindo-se 1-3 em 1-2 resulta:

$$
\begin{aligned}
& \sigma_{r} \cdot r \cdot d \phi \cdot z-\left(\sigma_{r}+\frac{d \sigma}{d r} d r\right) \cdot(r+d r) \cdot d \phi \cdot z+2 \sigma_{c} \cdot d r \cdot z \cdot \sin \frac{d \phi}{2}=0 \\
& \quad \text { e, cancelando-se } z, \text { obtem-se: }
\end{aligned}
$$

$$
\sigma_{r} \cdot r \cdot d \phi-\left(\sigma_{r}+\frac{d \sigma}{d r} d r\right) \cdot(r+d r) \cdot d \phi+2 \sigma_{c} \cdot d r \cdot \sin \frac{d \phi}{2}=0
$$

No limite para $d \phi \rightarrow 0$, tem-se

$$
\sin \frac{d \phi}{2} \approx \frac{d \phi}{2}
$$

e, portanto, resulta em

$$
\sigma_{r} \cdot r-\left(\sigma_{r}+\frac{d \sigma}{d r} d r\right) \cdot(r+d r)+\sigma_{c} \cdot d r=0
$$

$\mathrm{Ou}$

$$
\sigma_{r} \cdot r+\sigma_{c} \cdot d r-\left(\sigma_{r} \cdot r+\sigma_{r} \cdot d r+\frac{d \sigma_{r}}{d r} \cdot d r \cdot r+\frac{d \sigma_{r}}{d r} \cdot d r \cdot d r\right)=0
$$

que simplificando vem

$$
\sigma_{r} \cdot r+\sigma_{c} \cdot d r-\sigma_{r} \cdot r-\sigma_{r} \cdot d r+d \sigma_{r} \cdot r+d \sigma_{r} \cdot d r=0
$$

O último termo da equação, por ser de ordem superior, no processo do limite pode ser abandonado em presença dos demais e, portanto,

$$
\sigma_{c} d r-\sigma_{r} d r-d \sigma_{r} r=0
$$

ou, ainda

Compatibilidade Geométrica:

$$
\sigma_{c}-\sigma_{r}-r \cdot \frac{d \sigma_{r}}{d r}=0
$$

$$
\begin{gathered}
\epsilon_{r}=\frac{\delta}{L}=\frac{\left(W+\frac{d W}{d r} \cdot d r\right)-W}{d r} \\
\epsilon_{r}=\frac{d W}{d r} \\
\epsilon_{c}=\frac{2 \pi(r+W)-2 \pi r}{2 \pi r}
\end{gathered}
$$




$$
\epsilon_{c}=\frac{W}{r}
$$

Propriedades do Material (Lei de Hooke):

$$
\begin{aligned}
\epsilon_{r} & =\frac{1}{E}\left(\sigma_{r}-\nu \sigma_{c}-\nu \sigma_{x}\right) \\
\epsilon_{c} & =\frac{1}{E}\left(\sigma_{c}-\nu \sigma_{r}-\nu \sigma_{x}\right) \\
\epsilon_{x} & =\frac{1}{E}\left(\sigma_{x}-\nu \sigma_{r}-\nu \sigma_{c}\right)
\end{aligned}
$$

Para o deslocamento axial, tem-se:

$$
\epsilon_{x}=0
$$

e, assim,

$$
\sigma_{x}=\nu\left(\sigma_{r}+\sigma_{c}\right)
$$

que, substituindo-se nas equações para $\epsilon_{r}$ e $\epsilon_{c}$ temos:

$$
\begin{gathered}
\epsilon_{r}=\frac{1}{E}\left(\sigma_{r}-\nu \sigma_{c}-\nu^{2}\left(\sigma_{r}+\sigma_{c}\right)\right) \\
\sigma_{c}=\frac{1}{E}\left(-\nu \sigma_{r}+\sigma_{r}-\nu^{2}\left(\sigma_{r}+\sigma_{c}\right)\right)
\end{gathered}
$$

Assim, temos o sistema de equações

$$
\begin{aligned}
& \sigma_{r}=\epsilon_{r} E+\nu \sigma_{c}+\nu^{2}\left(\sigma_{r}+\sigma_{c}\right) \\
& \sigma_{c}=\epsilon_{c} E+\nu \sigma_{r}+\nu^{2}\left(\sigma_{r}+\sigma_{c}\right)
\end{aligned}
$$

que resolvendo-se fornece

$$
\begin{aligned}
& \sigma_{r}=\frac{E}{(1+\nu)(1-2 \nu)}\left((1-\nu) \epsilon_{r}+\nu \epsilon_{c}\right) \\
& \sigma_{c}=\frac{E}{(1+\nu)(1-2 \nu)}\left(\nu \epsilon_{r}+(1-\nu) \epsilon_{c}\right)
\end{aligned}
$$

Mas,considerando-se as definições de $\epsilon_{r}$ e $\epsilon_{c}$

$$
\begin{gathered}
\sigma_{r}=\frac{E}{(1+\nu)(1-2 \nu)}\left[(1-\nu) \frac{d W}{d r}+\nu \frac{W}{r}\right] \\
\sigma_{c}=\frac{E}{(1+\nu)(1-2 \nu)}\left[\nu \frac{d W}{d r}+(1-\nu) \frac{W}{r}\right]
\end{gathered}
$$


Devemos agora aplicar o equilíbrio, dado pela equação (1-4): Resolvendo o sistema encontramos a equação diferencial dada por:

$$
\frac{d^{2} W}{d r^{2}}+\frac{1}{r} \frac{d W}{d r}-\frac{W}{r^{2}}=0
$$

A solução da equação em 1-10 obtida da combinação linear das funções.

$$
W(r)=A_{1} r+\frac{A_{2}}{r}
$$

cuja solução resulta da imposição de contorno.

A partir da solução da equação diferencial consideram-se dois casos principais. O primeiro, mais genérico, considera o efeito da pressão interna e externa, já o segundo será um caso particular do primeiro com pressão interna somente.

\subsection{1}

\section{Pressão interna e pressão externa}

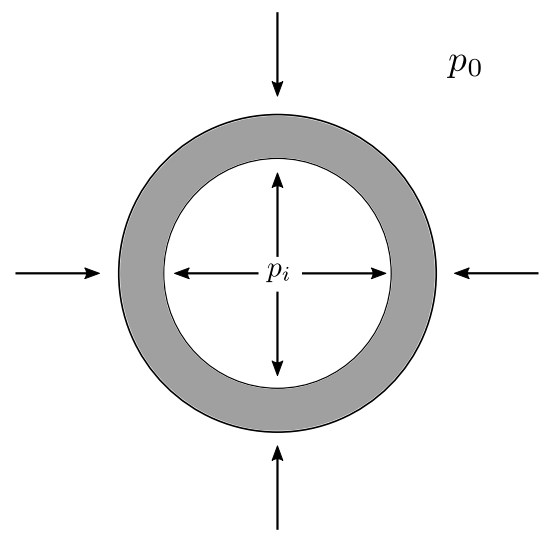

Figura 1.2: Seção reta de um cilindro sob pressão interna e externa

Quando submetido às pressões - internas e externas - têm-se as condições de contorno

$$
\begin{gathered}
\sigma_{r}\left(r_{i}\right)=-p_{i} \\
\sigma_{r}\left(r_{0}\right)=-p_{0}
\end{gathered}
$$

Da equação 1-5 tem-se então,

$$
\epsilon_{r}=\frac{d W}{d r}=\frac{d}{d r}\left(A_{1} r+A_{2} r^{-1}\right)=A_{1}-\frac{A_{2}}{r^{2}}
$$

Também da equção 1-6 pode-se escrever

$$
\epsilon_{c}=\frac{W}{r}=\left(A_{1} r+\frac{A_{2}}{r}\right) \cdot \frac{1}{r}=A_{1}+\frac{A_{2}}{r^{2}}
$$


Substituindo-se na equação 1-8 as equações acima 1-14 e 1-15:

$$
\sigma_{r}=\frac{E}{(1+\nu)(1-2 \nu)}\left[(1-\nu)\left(A_{1}-\frac{A_{2}}{r^{2}}\right)+\nu\left(A_{1}+\frac{A_{2}}{r^{2}}\right)\right]
$$

$\mathrm{Ou}$

$$
\sigma_{r}=\frac{E}{(1+\nu)(1-2 \nu)}\left[A_{1}-(1-2 \nu) \cdot \frac{A_{2}}{r^{2}}\right]
$$

Das condições de contorno resulta, então,

$$
\begin{gathered}
\sigma_{r}\left(r_{i}\right)=-p_{i}=\frac{E}{(1+\nu)(1-2 \nu)}\left[A_{1}-(1-2 \nu) \frac{A_{2}}{r_{i}^{2}}\right] \\
\sigma_{r}\left(r_{o}\right)=-p_{o}=\frac{E}{(1+\nu)(1-2 \nu)}\left[A_{1}-(1-2 \nu) \frac{A_{2}}{r_{o}^{2}}\right]
\end{gathered}
$$

ou , ainda, da equação 1-17

$$
A_{1}=(1-2 \nu) \frac{A_{2}}{r_{i}^{2}}-\frac{p_{i}(1+\nu)(1-2 \nu)}{E}
$$

e da equação 1-18.

$$
A_{2}=\frac{A_{1} E r_{o}^{2}}{(1-2 \nu)}+\frac{p_{o}(1+\nu) r_{o}^{2}}{E}
$$

Do sistema formado pelas equações 1-19 e 1-20 obtêm-se as expressões das constantes $A_{1}$ e $A_{2}$ em função de $p_{o}$ e $p_{i}$. Desta forma,

$$
\begin{gathered}
A_{1}=\frac{(1+\nu)(1-2 \nu)}{E} \frac{p_{i} r_{i}^{2}-p_{o} r_{o}^{2}}{r_{o}^{2}-r_{i}^{2}} \\
A_{2}=\frac{1+\nu}{E} \frac{\left(p_{i}-p_{o}\right) r_{i}^{2} r_{o}^{2}}{r_{o}^{2}-r_{i}^{2}}
\end{gathered}
$$

As expressões para $A_{1}$ e $A_{2}$ obtidos, explicitados nas equações 1-21 e 1-22, são substituidos na equação equação 1-16, resultando

$$
\sigma_{r}=\frac{p_{i} r_{i}^{2}-p_{o} r_{o}^{2}}{r_{o}^{2}-r_{i}^{2}}-\frac{1}{r^{2}} \frac{\left(p_{i}-p_{o}\right) r_{i}^{2} r_{o}^{2}}{r_{o}^{-} r_{i}^{2}}
$$

Da mesma forma, a expressão da tensão circunferencial é obtida substituindo-se em deve-se utilizar a equação 1-9 substituindo pelas equações

$$
\begin{aligned}
& \text { 1-14 e 1-15] } \sigma_{c}=\frac{E}{(1+\nu)(1-2 \nu)}\left[A_{1}+\frac{A_{2}}{r^{2}}(1-2 \nu)\right] \\
& \sigma_{c}=\frac{E}{(1+\nu)(1-2 \nu)}\left[\frac{(1+\nu)(1-2 \nu)}{E} \frac{p_{i} r_{i}^{2}-p_{o} r_{o}^{2}}{r_{o}^{2}-r_{i}^{2}}+\frac{(1-2 \nu)}{r^{2}} \frac{(1+\nu)}{E} \frac{\left(p_{i}-p_{o}\right) r_{i}^{2} r_{o}^{2}}{r_{o}^{2}-r_{i}^{2}}\right] \\
& \text { Portanto: } \quad \sigma_{c}=\frac{p_{i} r_{i}^{2}-p_{o} r_{o}^{2}}{r_{o}^{2}-r_{i}^{2}}+\frac{1}{r^{2}} \frac{\left(p_{i}-p_{o}\right) r_{i}^{2} r_{o}^{2}}{r_{o}^{2}-r_{i}^{2}}
\end{aligned}
$$


Como caso particular as expressões para as tensões normais devido a ação de pressão interna resulta das equações 1-23 e 1-25 na forma,

$$
\begin{aligned}
\sigma_{r} & =\frac{p_{i} r_{i}^{2}}{r_{o}^{2}-r_{i}^{2}}\left(1-\frac{r_{o}^{2}}{r^{2}}\right) \\
\sigma_{c} & =\frac{p_{i} r_{i}^{2}}{r_{o}^{2}-r_{i}^{2}}\left(1+\frac{r_{o}^{2}}{r^{2}}\right)
\end{aligned}
$$




\section{2}

\section{Solução para Esferas}

Nesta seção considera-se uma esfera de raio interno $r_{i}$ e raio externo $r_{o}$, submetidos às pressões interna $p_{i}$ e externa $p_{o}$,

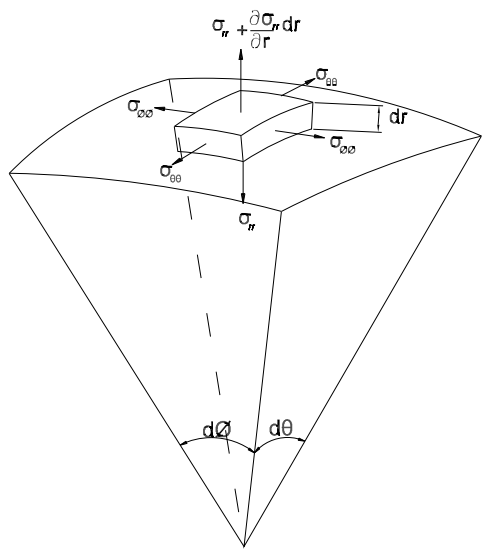

Figura 1.3: Elemento infinitesimal tridimensional

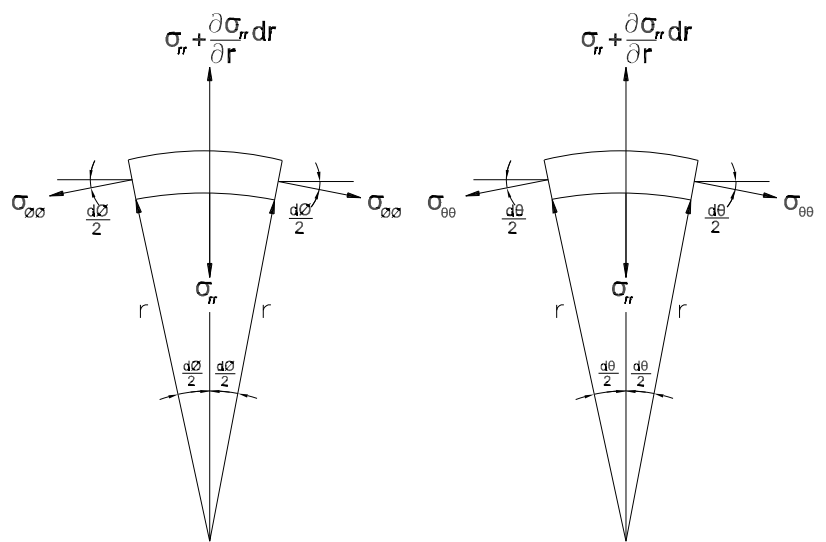

Figura 1.4: Elemento infinitesimal plano

Da figura 1.4 considerando-se o equilíbrio de forças na direção radial, obtem-se

$$
\begin{aligned}
& \left(\sigma_{r r}+\frac{\partial \sigma_{r r}}{\partial r} d r\right) \cdot(r+d r) d \theta(r+d r) d \phi-\sigma_{r r} r d \theta r d \phi \\
& \quad-2 \sigma_{\phi \phi} r d \theta d r \sin \left(\frac{d \phi}{2}\right)-2 \sigma_{\theta \theta} r d \phi d r \sin \left(\frac{d \theta}{2}\right)=0
\end{aligned}
$$

e, para pequenos ângulos, a equação resulta: 


$$
\begin{aligned}
\left(\sigma_{r r}+\frac{\partial \sigma_{r r}}{\partial r} d r\right) \cdot & (r+d r) d \theta(r+d r) d \phi-\sigma_{r r} r d \theta r d \phi \\
& -2 \sigma_{\phi \phi} r d \theta d r\left(\frac{d \phi}{2}\right)-2 \sigma_{\theta \theta} r d \phi d r\left(\frac{d \theta}{2}\right)=0
\end{aligned}
$$

ou ainda

$$
\begin{aligned}
\left(\sigma_{r r}+\frac{\partial \sigma_{r r}}{\partial r} d r\right) \cdot\left(r^{2}+2 r d r+d r^{2}\right)-\sigma_{r r} r^{2} & \\
& -\sigma_{\phi \phi} r d r-\sigma_{\theta \theta} r d r=0
\end{aligned}
$$

Considerando-se no processo limite $d r \rightarrow 0$ e eliminando-se os termos de ordem superior resulta

$$
r\left[r \frac{\partial \sigma_{r r}}{\partial r}+r\left(2 \sigma_{r r}-\sigma_{\phi \phi}-\sigma_{\theta \theta}\right)\right]=0
$$

As equações de compatibilidade geométrica, relativas aos deslocamentos radial $W$ e tangencial $U$, tem-se

$$
\begin{aligned}
\epsilon_{r} & =\frac{d W}{d r} \\
\epsilon_{c} & =\frac{W}{r} \\
\epsilon_{z} & =\frac{d W}{d z}
\end{aligned}
$$

Da equação constitutiva tem-se, então,

$$
\begin{aligned}
\epsilon_{r r} & =\frac{1}{E}\left[\sigma_{r r}-\nu\left(\sigma_{\theta \theta}+\sigma_{\phi \phi}\right)\right] \\
\epsilon_{\theta \theta} & =\frac{1}{E}\left[\sigma_{\theta}-\nu\left(\sigma_{r r}+\sigma_{\theta \theta}\right)\right] \\
\epsilon_{\phi \phi} & =\frac{1}{E}\left[\sigma_{\phi \phi}-\nu\left(\sigma_{r r}+\sigma_{\theta \theta}\right)\right]
\end{aligned}
$$

onde $W$ é o deslocamento radial da esfera, $\nu$ é o coeficiente de poisson e E o módulo de elasticidade do material.

Para esta geometria as deformação tangenciais são iguais, por simetria, e tem-se:

$$
\sigma_{\phi \phi}=\sigma_{\theta \theta}
$$

Considerando as equações 1-34 e 1-35 as tensões $\sigma_{r r}$ e $\sigma_{\theta \theta}$ resultam na expressão

$$
\sigma_{r r}=E \frac{\partial W}{\partial r}+2 \nu \sigma_{\theta \theta}=\frac{E}{(1-2 \nu)(1+\nu)}\left[(1-\nu) \frac{\partial W}{\partial r}+2 \nu \frac{W}{r}\right]
$$




$$
\sigma_{\theta \theta}=\sigma_{\phi \phi}=\frac{E}{(1-2 \nu)(1+\nu)}\left[\nu \frac{\partial W}{\partial r}+\frac{\partial W}{\partial r}\right]
$$

Voltando à equação de equilíbrio em 1-30 as equações 1-36 e 1-37 obtemse a equação, relativa ao deslocamento radial, na forma

$$
r \frac{\partial^{2} W}{\partial r^{2}}+2 \frac{\partial W}{\partial r}-2 \frac{W}{r}=0
$$

em que a solução é dada por:

$$
W(r)=A r+\frac{B}{r^{2}}
$$

onde $A$ e $B$ são constantes determinadas pelas condições de contorno do problema. Como condições a tensão radial tem o valor igual a pressão interna no raio interno e o valor negativo igual a pressão externa, para o raio externo. Assim,

$$
\begin{gathered}
\left.\sigma_{r r}\right|_{r=r_{i}}=-p_{i}=\frac{E}{(1-2 \nu)(1+\nu)}\left[\left.(1-\nu) \frac{\partial W}{\partial r}\right|_{r=r_{i}}+\left.2 \nu \frac{W}{r}\right|_{r=r_{i}}\right] \\
\left.\sigma_{r r}\right|_{r=r_{0}}=-p_{0}=\frac{E}{(1-2 \nu)(1+\nu)}\left[\left.(1-\nu) \frac{\partial W}{\partial r}\right|_{r=r_{0}}+\left.2 \nu \frac{W}{r}\right|_{r=r_{0}}\right]
\end{gathered}
$$

o que resulta em

$$
\begin{gathered}
\left.\frac{W}{r}\right|_{r=r_{i}}=A+\left.\frac{B}{r_{i}^{3}} \quad \frac{W}{r}\right|_{r=r_{0}}=A+\frac{B}{r_{0}^{3}} \\
\left.\frac{\partial W}{\partial r}\right|_{r=r_{i}}=A-\left.2 \frac{B}{r_{i}^{3}} \quad \frac{\partial W}{\partial r}\right|_{r=r_{0}}=A-2 \frac{B}{r_{0}^{3}}
\end{gathered}
$$

Substituindo-se os resultados às equações 1-42 e 1-43 em 1-40 e 1-41 as constantes $A$ e $B$ são assim obtidas

$$
\begin{gathered}
A=\frac{(1-2 \nu)}{E\left(r_{0}^{3}-r_{i}^{3}\right)}\left[p_{i} r_{i}^{3}-p_{o} r_{0}^{3}\right] \\
B=\frac{(1+\nu)}{2 E} \frac{r_{i}^{3} r_{0}^{3}}{\left(r_{0}^{3}-r_{i}^{3}\right)}\left[p_{i}-p_{0}\right]
\end{gathered}
$$

que substituidas as equações 1-44 e 1-45 na equação 1-39, tem-se o deslocamento radial na forma analítica

$$
W(r)=\frac{(1-2 \nu)}{E\left(r_{0}^{3}-r_{i}^{3}\right)}\left[p_{i} r_{i}^{3}-p_{o} r_{0}^{3}\right] r+\frac{(1+\nu)}{2 E} \frac{r_{i}^{3} r_{0}^{3}}{\left(r_{0}^{3}-r_{i}^{3}\right)}\left[p_{i}-p_{0}\right] \frac{1}{r^{2}}
$$

Da mesma forma substituindo-se as equações 1-44 e 1-45 nas expressões às equações 1-36 e 1-37 obtem-se as componentes de tensões: 


$$
\begin{gathered}
\sigma_{r r}(r)=-\frac{p_{i} r_{i}^{3}}{r^{3}}\left[\frac{r_{0}^{3}-r^{3}}{r_{0}^{3}-r_{i}^{3}}\right]+\frac{p_{0} r_{0}^{3}}{r^{3}}\left[\frac{r_{i}^{3}-r^{3}}{r_{0}^{3}-r_{i}^{3}}\right] \\
\sigma_{\theta \theta}(r)=\sigma_{\phi \phi}(r)=\frac{p_{i} r_{i}^{3}}{2 r^{3}}\left[\frac{r_{0}^{3}+2 r^{3}}{r_{0}^{3}-r_{i}^{3}}\right]-\frac{p_{0} r_{0}^{3}}{2 r^{3}}\left[\frac{r_{i}^{3}+2 r^{3}}{r_{0}^{3}-r_{i}^{3}}\right]
\end{gathered}
$$




\section{3}

\section{Disco Girante}

Um cilindro girante em torno do seu eixo geométrico com uma velocidade angular $\omega$ é obtido considerando-se o problema axissimétrico.

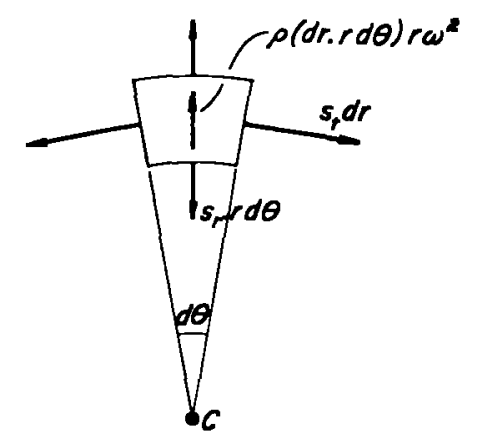

Figura 1.5: Elemento infinitesimal do disco girante

A condição de equilíbrio é obtida considerando-se a força centrífuga infinitesimal, na forma

$$
d F_{c}=a_{c} \cdot d m=w^{2} \cdot r \cdot \rho \cdot d r \cdot r \cdot d \theta
$$

Do equilíbrio estático tem-se que

$$
\sum F_{\hat{r}}=0
$$

ou, ainda

$$
\omega^{2} r \rho d r r d \theta+\left[\sigma_{r} r d \theta+\frac{d}{d r}\left(\sigma_{r} r d \theta\right) d r\right]-\sigma_{r} r d \theta-2 \sigma_{c} d r \sin \frac{d \theta}{2}=0
$$

que, simplificndo a álgebra resulta em

$$
\frac{d}{d r}\left(r \sigma_{r}\right)-\sigma_{c}+\rho w^{2} r^{2}=0
$$

As equações de compatibilidade Assim como feito acima na solução de Lamé, temos as equações 1 e 2. Dada por:

A condição constitutiva é

$$
\begin{aligned}
\epsilon_{r} & =\frac{\partial W}{\partial r} \\
\epsilon_{c} & =\frac{W}{r}
\end{aligned}
$$

$$
\epsilon_{r}=\frac{1}{E}\left(\sigma_{r}-\nu \sigma_{c}-\nu \sigma_{x}\right)
$$




$$
\epsilon_{c}=\frac{1}{E}\left(\sigma_{c}-\nu \sigma_{r}-\nu \sigma_{x}\right)
$$

$\operatorname{com} \sigma_{x}=0$, e fazendo os resultados acima em função de $W$, então,

ou ainda, pela equação (1-55):

$$
\begin{gathered}
\frac{\partial W}{\partial r}=\frac{1}{E}\left(\sigma_{r}-\nu \sigma_{c}\right) \\
\frac{W}{r}=\frac{1}{E}\left(\sigma_{c}-\nu \sigma_{r}\right)
\end{gathered}
$$

$$
W=\frac{r}{E} \sigma_{c}-\frac{\nu r}{E} \sigma_{r}
$$

Derivando a equação número (1-56) acima e igualando a equação (1-54), resulta:

$$
\frac{d W}{d r}=\frac{1}{E}\left(\sigma_{c}-\nu \sigma_{r}\right)+\frac{r}{E}\left(\frac{d \sigma_{c}}{d r}-\frac{\nu d \sigma_{r}}{d r}\right)
$$

Igualando a equação (1-57) com (1-54), tem-se

$$
\frac{d \sigma_{c}}{d r}-\nu \frac{d \sigma_{r}}{d r}+\left(\frac{1+\nu}{r}\right)\left(\sigma_{c}-\sigma_{r}\right)=0
$$

Pela regra da cadeia, tem-se

$$
\frac{d}{d r}\left(r \sigma_{r}\right)=\sigma_{r}+r \frac{d \sigma_{r}}{d r}
$$

$\log \mathrm{e}$

$$
\frac{d \sigma_{r}}{d r}=\frac{1}{r}\left[\frac{d}{d r}\left(r \quad \sigma_{r}\right)-\sigma_{r}\right]
$$

Substituindo a equação 1-60 em 1-58 tem-se: Portanto:

$$
\frac{d \sigma_{c}}{d r}-\frac{\nu}{r}\left[\frac{d}{d r}\left(r \sigma_{r}\right)-\sigma_{r}\right]+\left(\frac{1+\nu}{r}\right)\left(\sigma_{c}-\sigma_{r}\right)=0
$$

Utilizando a equação de equilíbrio estático, número (1-51), podemos isolar a tensão circunferencial por:

$$
\sigma_{c}=\frac{d}{d r}\left(r \sigma_{r}\right)+\rho \omega^{2} r^{2}
$$

Derivando a equação número 1-62 acima resulta

$$
\frac{d \sigma_{c}}{d r}=\frac{d^{2}}{d r^{2}}\left(r \sigma_{r}\right)+2 \rho \omega^{2} r
$$

Do sistema composto pelas equações 1-60), 1-61) e (1-62), resulta

$$
\frac{d^{2}}{d r^{2}}\left(r \sigma_{r}\right)+2 \rho \omega^{2} r-\frac{\nu}{r} \frac{d}{d r}\left(r \sigma_{r}\right)+\frac{\nu}{r} \sigma_{r}+\left(\frac{1+\nu}{r}\right) \cdot\left(\frac{d}{d r}\left(r \sigma_{r}\right)+\rho \omega^{2} r^{2}-\sigma_{r}\right)=0
$$

Utilizando "linha"como derivada em relação a r e expandindo a equação acima, temos: 


$$
r^{2} \cdot\left(r \sigma_{r}\right)^{\prime \prime}+r \cdot\left(r \sigma_{r}\right)^{\prime}-\left(r \sigma_{r}\right)+(3+\nu) \cdot \rho \omega^{2} r^{3}=0
$$

A equação diferencial acima é linear, porém seus coeficientes não são constantes.

A solução da equação em (1-65) é da forma,

$$
\left(r \sigma_{r}\right)=r^{n}
$$

Onde $n$ é um expoente desconhecido. logo,

$$
\begin{gathered}
\left(r \sigma_{r}\right)^{\prime}=\left(r^{n}\right)^{\prime}=n \cdot r^{n-1} \\
\left(r \sigma_{r}\right)^{\prime \prime}=\left(r^{n}\right)^{\prime \prime}=\left(n \cdot r^{n-1}\right)^{\prime}=n \cdot(n-1) \cdot r^{n-2}
\end{gathered}
$$

Substituindo na equação homogênea da equação (1-65) tem-se

$$
[n \cdot(n-1)+n-1] \cdot r^{n}=0
$$

Sabendo que $r^{n}$ não pode ser igual a zero, ou a primeira parcela da equação acima é zero ou a segunda, então

$$
\begin{gathered}
n \cdot(n-1)+(n-1)=0 \\
(n+1) \cdot(n-1)=0 \rightarrow n= \pm 1
\end{gathered}
$$

Logo, a solução da equação homogênea é:

$$
\left(r \sigma_{r}\right)=C_{1} \cdot r+\frac{C_{2}}{r}
$$

A solução particular será da forma $A \cdot r^{3}$, então

$$
\left(r \sigma_{r}\right)=C_{1} \cdot r+\frac{C_{2}}{r}+A \cdot r^{3}
$$

Portanto, suas derivadas:

$$
\begin{gathered}
\left(r \sigma_{r}\right)^{\prime}=C_{1}-\frac{C_{2}}{r^{2}}+3 \cdot A \cdot r^{2} \\
\left(r \sigma_{r}\right)^{\prime \prime}=\frac{2 \cdot C_{2}}{r^{3}}+6 \cdot A \cdot r
\end{gathered}
$$

Substituindo as equações 1-69, 1-70 e 1-71) na equação diferencial (1-65) determina-se a constante $A$, dada por:

$$
A=-\frac{(3+\nu)}{8} \cdot \rho \cdot \omega^{2}
$$

Substituindo a constante $A$, da equação (1-72 em (1-69), então

$$
\sigma_{r}=C_{1}+\frac{C_{2}}{r^{2}}-\frac{(3+\nu)}{8} \cdot \rho \cdot \omega^{2} \cdot r^{2}
$$


Substituindo a equação acima 1-73 na equação de equilíbrio estático número 1-51 determina-se a tensão circunferencial dada por:

$$
\sigma_{c}=\frac{d}{d r}\left(r \sigma_{r}\right)+\rho \cdot \omega^{2} \cdot r^{2}
$$

Aplicando a equação número (1-73) em (1-74), tem-se

$$
\sigma_{c}=C_{1}-\frac{C_{2}}{r^{2}}-\frac{(1+3 \cdot \nu)}{8} \cdot \rho \cdot \omega^{2} \cdot r^{2}
$$

Substituindo as equações de tensão radial e circunferencial números (1-73) e 1-75), respectivamente, na equação de deslocamento radial 1-55 obtem-se

$$
W=\frac{r}{E}\left[(1-\nu) \cdot C_{1}-(1+\nu) \cdot \frac{C_{2}}{r^{2}}-\frac{1-\nu^{2}}{8} \cdot \rho \cdot \omega^{2} \cdot r^{2}\right]
$$




\subsection{1}

\section{Disco Sólido com pressão externa e rotação}

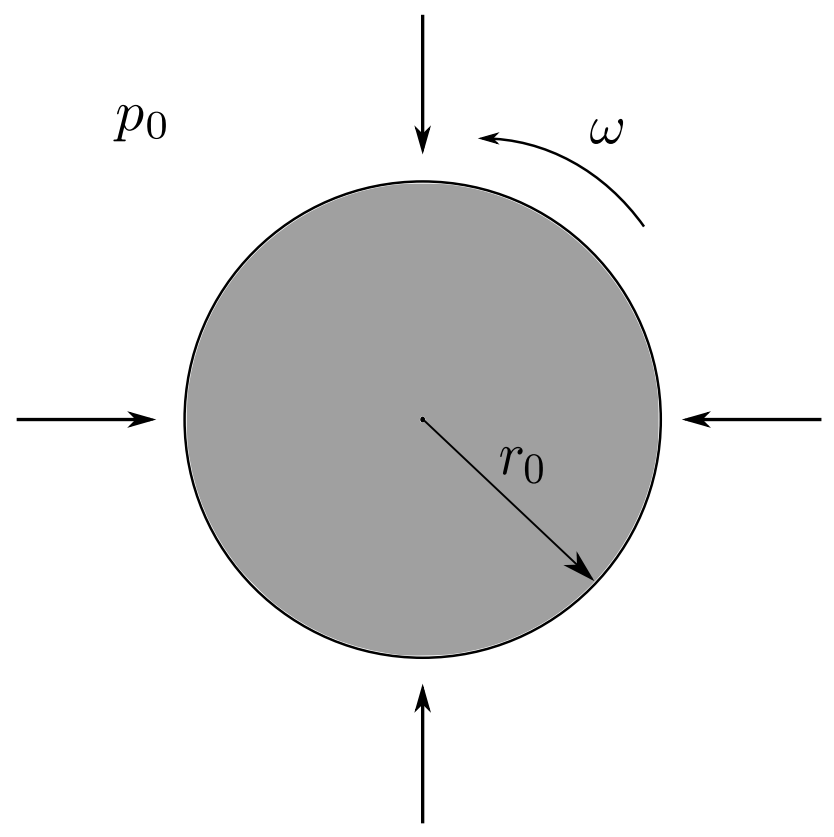

Ao analisar a equação 1-73

$$
\sigma_{r}=C_{1}+\frac{C_{2}}{r^{2}}-\frac{(3+\nu)}{8} \cdot \rho \cdot \omega^{2} \cdot r^{2}
$$

percebe-se que em $r=0$, a fim de evitar indeterminação matemática, a tensão radial é constante. Portanto, $C_{2}$ precisa, necessariamente, ser igual a zero. Fisicamente, não se pode aceitar que a tensão radial tenda a infinito à medida que o raio se aproxime a zero, então

$$
C_{2}=0
$$

Pela condição de contorno tem-se

$$
\sigma_{r}\left(r_{0}\right)=-p_{0}
$$

Substituindo as equações 1-78 e 1-79 na equação número 1-77 determinase o valor da constante $C_{1}$.

$$
C_{1}=\frac{3+\nu}{8} \cdot \rho \cdot \omega^{2} \cdot r_{0}^{2}-p_{0}
$$

Pela equação número 1-73, pode-se obter a tensão radial:

$$
\sigma_{r}=-p_{0} \frac{3+\nu}{8} \cdot \rho \cdot \omega^{2}\left(r_{0}^{2}-r^{2}\right)
$$

e pela equação número 1-75 pode-se determinar a tensão circunferencial:

$$
\sigma_{c}=-p_{0}+\frac{3+\nu}{8} \rho \omega^{2}\left(r_{0}^{2}-\frac{1+3 \nu}{3+\nu} \cdot r^{2}\right)
$$


Substituindo as constantes $C_{1}$ e $C_{2}$ na equação do deslocamento radial 1-76 tem-se

$$
W=-p_{0} r \frac{(1-\nu)}{E}+\frac{(1-\nu) \rho \omega^{2} r}{8 E}\left[(3+\nu) r_{0}^{2}-(1+\nu) r^{2}\right]
$$

Uma observação interessante nesse caso é a tensão no centro do disco, que caracterizará um estado hisdrostático de tensão de duas dimensões, por compatibilidade:

$$
\sigma_{\text {centro }}=\sigma_{r}(0)=\sigma_{c}(0)
$$

Para o caso de $p_{0}=0$ :

$$
\sigma_{\text {centro }}=\frac{3+\nu}{8} \rho\left(\omega r_{0}\right)^{2}
$$




\subsection{2}

\section{Disco com furo no centro e rotação}

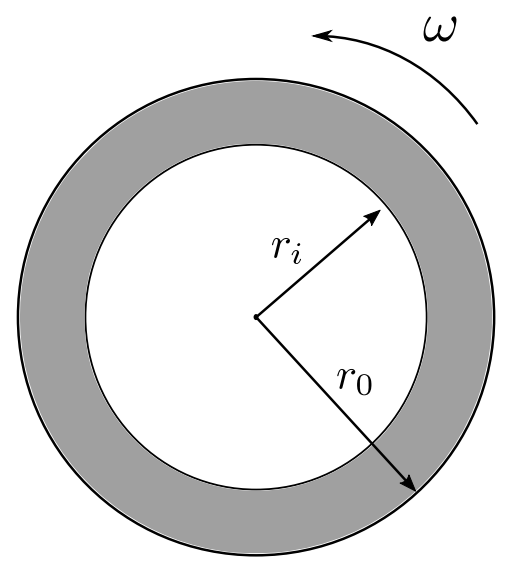

Pelas condições de contorno do problema problema com $\omega \neq 0$ tem-se

$$
\left\{\begin{array}{l}
\sigma_{r}\left(r_{o}\right)=0 \\
\sigma_{r}\left(r_{i}\right)=0
\end{array}\right.
$$

Substituindo as duas condições de contorno acima na equação número 1-73 obtem-se um sistema composto por duas equações e duas incógnitas $\left(C_{1}\right.$ e $\left.C_{2}\right)$, assim sua solução é garantida e dada por:

$$
\begin{gathered}
C_{1}=\frac{3+\nu}{8} \rho \omega^{2}\left(r_{o}^{2}+r_{i}^{2}\right) \\
C_{2}=-\frac{3+\nu}{8} \rho \omega^{2} r_{i}^{2} r_{o}^{2}
\end{gathered}
$$

Substituindo as equações 1-86 e 1-85 em 1-73 determina-se a tensão radial, assim

$$
\sigma_{r}=\frac{3+\nu}{8} \rho \omega^{2}\left(r_{o}^{2}+r_{i}^{2}-\frac{r_{i}^{2} r_{o}^{2}}{r^{2}}-r^{2}\right)
$$

e pela equação 1-75 e substituindo $C_{1}$ e $C_{2}$ das equações 1-86 e 1-85 obtem-se a tensão circunferencial, dada por

$$
\sigma_{c}=\frac{3+\nu}{8} \rho \omega^{2}\left(r_{o}^{2}+r_{i}^{2}+\frac{r_{i}^{2} r_{o}^{2}}{r^{2}}-\frac{1+3 \nu}{3+\nu} r^{2}\right)
$$

Para o deslocamento radial deve-se substituir na equação 1-76 as equações 1-86 e 1-85, assim

$$
W=\frac{(1-\nu)(3+\nu)}{8 E} \rho \omega^{2} r\left[r_{o}^{2}+r_{i}^{2}+\left(\frac{1+\nu}{1-\nu}\right) \frac{r_{o}^{2} r_{i}^{2}}{r^{2}}-\left(\frac{1+\nu}{3+\nu}\right) r^{2}\right]
$$




\section{4}

\section{Resumo das soluções analíticas}

Nessa seção os principais resultados obtidos acima serão novamente apresentados, considerando as limitações do modelo no desenvolvimento analítico.

O primeiro tópico apresentado foi a solução de Lamé para cilindro, em que foi imposto deslocamento axial nulo no cálculo das equações constitutivas. Todavia, diferentemente da solução de Lamé, o desenvolvimento analítico para cilindros submetidos a carregamento de rotação apresenta condição de contorno livre. Assim, não se pode utilizar superposição entre a solução de Lamé e e a solução para cilindros com rotação, pois suas condições de contorno são distintas.

Para esferas não é considerado nenhuma restrição a deslocamento em seu desenvolvimento.

A tabela abaixo reúne as principais fórmulas desse capítulo considerando apenas corpos cilíndricos. Vale ressaltar, que a temática do presente trabalho, apesar de considerar uma formulação capaz de incorporar a solução de esferas, desenvolve uma metodologia de elementos finitos restrita a cilindros, deixando o caso de esferas para um futuro trabalho. 


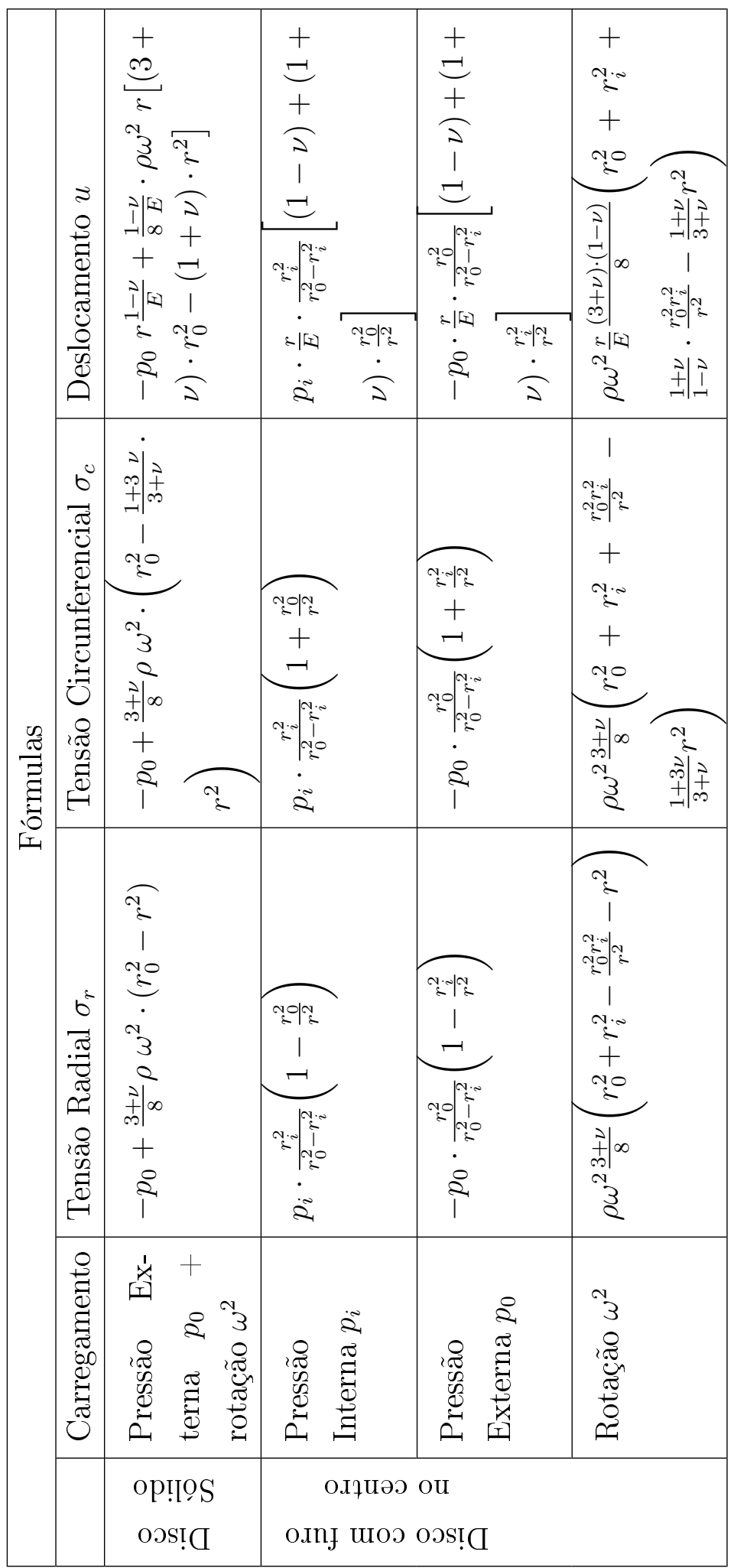




\section{2}

\section{Modelo de cascas}

No desenvolvimento do modelo de elementos finitos os conceitos de teoria de cascas finas devem ser aplicados de forma a obter-se expressões para os deslocamentos e as deformações, analiticamente.

\section{1}

\section{Introdução à teoria de cascas finas}

\subsection{1}

\section{Primeira forma fundamental}

Uma superfície pode ser descrita pelo sistema de coordenadas cartesiano $\left(\hat{X}_{1}, \hat{X}_{2}, \hat{X}_{3}\right)$ em termos dos parâmetros geométricos $\xi$ e $\theta$.

$$
\hat{X}_{i}=f_{i}(\xi, \theta), i=1,2,3
$$

onde $f_{i}$ é uma função com contra-domínio real e contínua das superfícies curvilíneas $\xi$ e $\theta$ que determina a família das curvas paramétricas da superfície.

$O$ vetor posição de um ponto $P$ da superfície é dado por

$$
\hat{X}=\hat{X}(\xi, \theta)=\sum_{i=1}^{3} f_{i}(\xi, \theta) \hat{e}_{i}
$$

onde $\hat{e}_{i}$ é o vetor unitário paralelo à coordenada $x_{i}$.

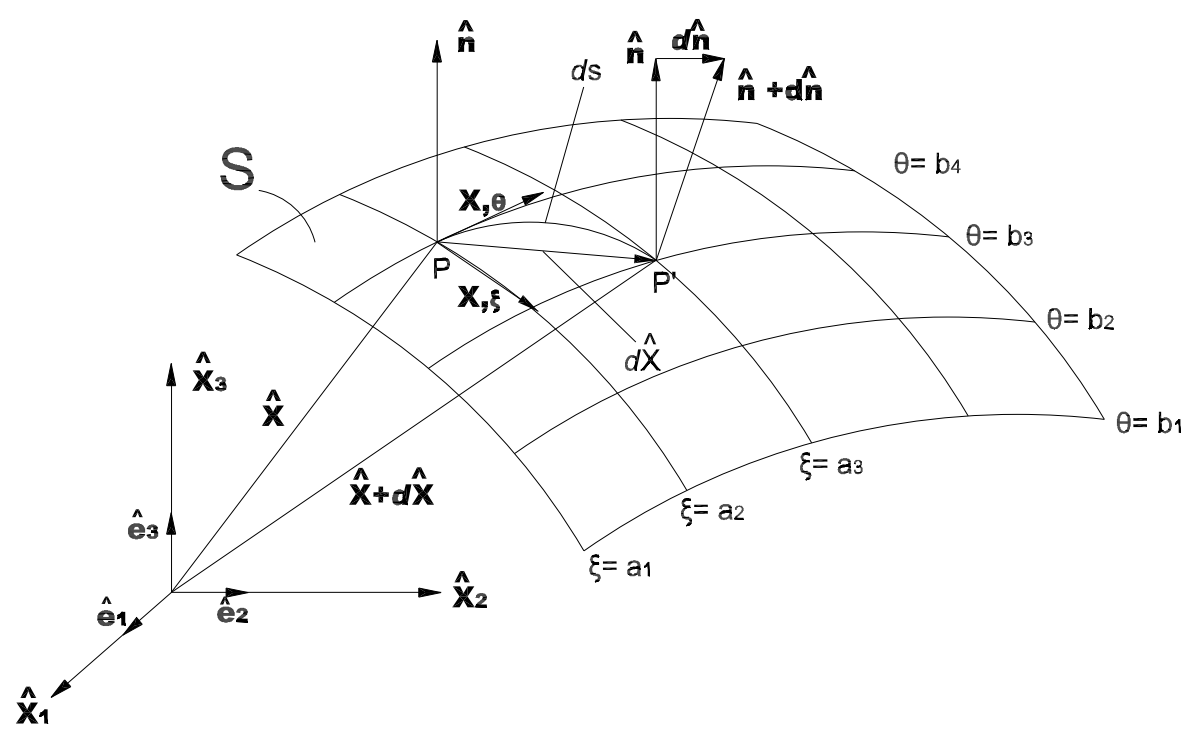

Figura 2.1: Superfície

Entre dois pontos $P$ e um $P^{\prime}$ temos: 


$$
d \hat{X}=\frac{\partial \hat{X}}{\partial \xi} d \xi+\frac{\partial \hat{X}}{\partial \theta} d \theta \equiv \hat{X}_{, \xi} d \xi+\hat{X}_{, \theta} d \theta
$$

Portanto, a magnitude da distância deslocada na superfície é:

$$
\begin{aligned}
(d s)^{2}=d \hat{X} \cdot d \hat{X} \equiv\left(\hat{X}_{, \xi} d \xi+\hat{X}_{, \theta} d \theta\right) & \cdot\left(\hat{X}_{, \xi} d \xi+\hat{X}_{, \theta} d \theta\right) \\
& =E(d \xi)^{2}+2 F(d \xi d \theta)+G(d \theta)^{2}
\end{aligned}
$$

Onde:

$$
E=\hat{X}_{, \xi} \cdot \hat{X}_{, \xi} \quad F=\hat{X}_{, \xi} \cdot \hat{X}_{, \theta} \quad G=\hat{X}_{, \theta} \cdot \hat{X}_{, \theta}
$$

A equação (2-3), com os coeficientes definidos na equação (2-5), é a conhecida primeira forma fundamental de uma superfície e os valores $F, E$ e $G$ são as primeiras magnitudes fundamentais.

Para o comprimento infinitesimal do arco em $\xi$ ao longo da curva constante em $\theta$, tem-se:

$$
(d s)^{2}=E(d \xi)^{2}+2 F(d \xi d \theta)+G(d \theta)^{2}
$$

Sendo constante o valor de $\theta$, sabe-se que $d \theta=0$.

$$
(d s)^{2}=E(d \xi)^{2}
$$

Portanto:

$$
d s_{\xi}=\sqrt{E} d \xi
$$

Analogamente, para o comprimento infinitesimal do arco em $\theta$ ao longo da curvatura constante em $\xi$, obtem-se:

$$
d s_{\theta}=\sqrt{G} d \theta
$$

Ao observar a equação (2-5), nota-se que o coeficiente $F$ tem valor nulo, isso porque os vetores $\hat{X}_{, \xi}$ e $\hat{X}_{, \theta}$ são ortogonais, e como consequência seu produto interno é zero.

$$
F=0
$$

Reescrevendo a equação (2-4) :

$$
(d s)^{2}=A_{1}^{2}(d \xi)^{2}+A_{2}^{2}(d \theta)^{2}
$$

Onde:

$$
A_{1}^{2}=E \quad A_{2}^{2}=G
$$

Para o próximo passo deve-se encontrar a curvatura e a segunda forma fundamental. 


\section{1 .2}

\section{Curvatura e Segunda forma fundamental}

Para cada $P$ pertencente à superfície existe um vetor unitário normal a curva denotado $\hat{n}$, que é normal aos vetores $\hat{X}_{, \xi}, \hat{X}_{, \theta}$.

Portanto:

$$
\hat{n}(\xi, \theta)=\frac{\hat{X}_{, \xi} \times \hat{X}_{, \theta}}{\left|\hat{X}_{, \xi} \times \hat{X}_{, \theta}\right|}
$$

Considerando um ângulo $\xi$ entre os vetores $\hat{X}_{, \xi}$ e $\hat{X}_{, \theta}$.

$$
\begin{gathered}
\left|\hat{X}_{, \xi} \times \hat{X}_{, \theta}\right|=\left|\hat{X}_{, \xi}\right|\left|\hat{X}_{, \theta}\right| \sin \xi \\
\hat{X}_{, \xi} \cdot \hat{X}_{, \theta}=\left|\hat{X}_{, \xi}\right|\left|\hat{X}_{, \theta}\right| \cos \xi
\end{gathered}
$$

Logo:

$$
\begin{aligned}
& \cos \xi=\frac{\hat{X}_{, \xi} \cdot \hat{X}_{, \theta}}{\left|\hat{X}_{, \xi}\right|\left|\hat{X}_{, \theta}\right|}=\frac{\hat{X}_{, \xi} \cdot \hat{X}_{, \theta}}{\sqrt{\hat{X}_{, \xi} \cdot \hat{X}_{, \xi}} \sqrt{\hat{X}_{, \theta} \cdot \hat{X}_{, \theta}}} \equiv \frac{F}{\sqrt{E G}} \\
& \sin \xi=\sqrt{\left(1-\cos ^{2} \xi\right)}=\sqrt{\left(1-\frac{F^{2}}{E G}\right)}=\sqrt{\frac{E G-F^{2}}{E G}}
\end{aligned}
$$

Portanto, a expressão final resulta em

$$
\hat{n}(\xi, \theta)=\frac{\hat{X}_{, \xi} \times \hat{X}_{, \theta}}{H}, \quad H \equiv \sqrt{E G-F^{2}}
$$

Adotando-se a convenção de que o vetor normal $\hat{n}(\xi, \theta)$ é positivo se apontar do lado côncavo para o convexoda curva e sendo $\hat{t}$ o vetor tangente a curva, o parâmetro $\hat{k}$ define curvatura, como:

$$
\hat{k}=\frac{d \hat{t}}{d s}
$$

O vetor curvatura é decomposto em vetores nas direções normal e tangencial à curva, i.e.,

$$
\hat{k}=\frac{d \hat{t}}{d s}=\hat{k}_{n}+\hat{k}_{t}
$$

Em oposição à referência adotada no vetor normal o vetor de curvatura normal, $\hat{k}_{n}$, será positivo quando do convexo para o côncavo, ou seja

$$
\hat{k}_{n}=-K_{n} \hat{n}
$$

onde $K_{n}$ é a curvatura normal. Então temos $\hat{t} \cdot \hat{n}=0$ e

$$
\frac{d(\hat{t} \cdot \hat{n})}{d s}=0
$$


$\mathrm{Ou}$

$$
\frac{d \hat{n}}{d s} \cdot \hat{t}=-\frac{d \hat{t}}{d s} \cdot \hat{t} \cdot \hat{n}
$$

Da equação 2-14 observa-se que

$$
\left(\hat{k}_{n} \cdot \hat{n}\right)=-K_{n}(\hat{n} \cdot \hat{n})=-K_{n}
$$

e da equação 2-13 esta resulta em

$$
k \cdot \hat{n}=\frac{d \hat{t}}{d s} \cdot \hat{n}=\hat{k}_{n} \cdot \hat{n}+\hat{k}_{t} \cdot \hat{n}=\hat{k}_{n} \cdot \hat{n}
$$

onde

$$
\hat{k}_{n}=\frac{d \hat{t}}{d s}
$$

Assim,

$$
K_{n}=-\left(\hat{k}_{n} \cdot \hat{n}\right)=-\left(\frac{d \hat{t}}{d s} \cdot \hat{n}\right)=\frac{d \hat{n}}{d s} \cdot \hat{t}
$$

com

$$
K_{n}=\frac{d \hat{n}}{d s} \cdot \hat{t}
$$

Sabendo-se que:

$$
\hat{t}=\frac{d \hat{X}}{d s}
$$

substituindo o resultado da equação 2-16 na equação 2-15 fornece

$$
K_{n}=\frac{d \hat{n}}{d s} \cdot \frac{d \hat{X}}{d s}=\frac{d \hat{n} \cdot d \hat{X}}{(d s)^{2}}=\frac{d \hat{n} \cdot d \hat{X}}{d \hat{X} \cdot d \hat{X}}
$$

Onde:

$$
\begin{array}{r}
d \hat{n}=\hat{n}_{, \xi} d \xi+\hat{n}_{, \theta} d \theta \\
d \hat{X}=\hat{X}_{, \xi} d \xi+\hat{X}_{, \theta} d \theta
\end{array}
$$

Portanto, substituindo as equações (2-18) e 2-19) na equação (2-17) resulta

$$
K_{n}=\frac{L(d \xi)^{2}+2 M(d \xi \quad d \theta)+N(d \theta)^{2}}{E(d \xi)^{2}+2 F(d \xi \quad d \theta)+G(d \theta)^{2}}
$$

Na equação acima o numerador é a sugubdaforma fundamental definida pelas magnitudes

$$
L=\hat{n}_{, \xi} \cdot \hat{X}_{, \xi} \quad 2 M=\hat{n}_{, \xi} \cdot \hat{X}_{, \theta}+\hat{n}_{, \theta} \cdot \hat{X}_{, \xi} \quad N=\hat{n}_{, \theta} \cdot \hat{X}_{, \theta}
$$

Como $\hat{n} \cdot \hat{X}_{, \xi}=0$, tem-se então

$$
L=-\hat{n} \cdot \hat{X}_{, \xi \xi} \quad M=-\hat{n} \cdot \hat{X}_{, \xi \theta} \quad N=-\hat{n} \cdot \hat{X}_{, \theta \theta}
$$

Na notação acima os subíndices são derivadas parciais na forma $\hat{X}_{, \xi \theta}=$ $\frac{\partial^{2} \hat{X}}{\partial \xi \partial \theta}$. 
Considerando-se uma direção $\left(\frac{d \theta}{d \xi}\right) \equiv \lambda$ com a qual a curvatura será máxima ou mínima, isto é :

$$
\begin{gathered}
\left(\frac{d \theta}{d \xi}\right) \equiv \lambda \\
K_{n}(\lambda)=\frac{L+2 M \lambda+N \lambda^{2}}{E+2 F \lambda+G \lambda^{2}}
\end{gathered}
$$

Desta forma deve-se determinar $\lambda$ tal que $\frac{d K_{n}}{d \lambda}=0$. De algumas manipulações algébricas tem-se que os valores extremos de $K_{n}\left(\lambda_{o ́ t i m o}\right)$ é dado por:

$\mathrm{Ou}$

$$
K_{n}=\frac{M+N \lambda_{\text {ótimo }}}{F+G \lambda_{\text {ótimo }}}=\frac{L+M \lambda_{\text {ótimo }}}{E+F \lambda_{\text {ótimo }}}
$$

$$
\left(M+N \lambda_{\text {ótimo }}\right) \cdot\left(E+F \lambda_{\text {ótimo }}\right)=\left(L+M \lambda_{\text {ótimo }}\right) \cdot\left(F+G \lambda_{\text {ótimo }}\right)
$$

onde $\lambda_{\text {ótimo }}$ resulta das raizes do polinômio

$$
(M G-N F) \lambda_{o ́ t i m o}^{2}+(L G-N E) \lambda_{\text {ótimo }}+(L F-M E)=0
$$

correspondentes a valores máximo e mínimo da curvatura $K_{n}$. Estes São descritos como curvaturas principais $K_{1}$ e $K_{2}$, associados aos raios de curvatura $R_{1}$ e $R_{2}$, onde:

$$
R_{i}=K_{i}^{-1}, \quad i=1,2
$$

Além de representarem a máxima e a mínima curvaturas pode-se demonstrar que direções correspondentes são ortogonais. Assim, considera-se $\xi$ seja o ângulo entre as direções $\frac{d \theta}{d \xi}$ e $\frac{\delta \theta}{\delta \xi}$. Do diferencial de posição ao longo dessa direções, tem-se pela equação $2-3$ :

$$
d \hat{X}=\hat{X}_{, \xi} d \xi+\hat{X}_{, \theta} d \theta \quad \delta \hat{X}=\hat{X}_{, \xi} \delta \xi+\hat{X}_{, \theta} \delta \theta
$$

Pela definição de produto escalar:

$$
\cos \xi=\frac{d \hat{X} \cdot \delta \hat{X}}{|d \hat{X}||\delta \hat{X}|} \equiv E \frac{d \xi}{d s} \frac{\delta \xi}{\delta s}+F\left(\frac{d \xi}{d s} \frac{\delta \theta}{\delta s}+\frac{d \theta}{d s} \frac{\delta \xi}{\delta s}\right)+G \frac{d \theta}{d s} \frac{\delta \theta}{\delta s}
$$

Com o ângulo $\xi=\pi / 2$, tem-se $\lambda_{1}=\frac{d \theta}{d \xi}$ e $\lambda_{2}=\frac{\delta \theta}{\delta \xi}$, utilizando a definição na equação 2-23. Substituindo, vem

$$
E+F\left(\lambda_{1}+\lambda_{2}\right)+G \lambda_{1} \lambda_{2}=0
$$

que fornece

$$
\begin{aligned}
\lambda_{1}+\lambda_{2} & =-\frac{(L G-N E)}{(M G-N F)} \\
\lambda_{1} \lambda_{2} & =\frac{(L F-M E)}{(M G-N F)}
\end{aligned}
$$


Nota-se, portanto que são ortogonais, isso porque substituindo as equações 2-31 e 2-32 valida-se a equação 2-30.

$$
\begin{aligned}
& E \frac{(M G-N F)}{(M G-N F)}-F \frac{(L G-N E)}{(M G-N F)}+G \frac{(L F-M E)}{(M G-N F)}=0 \\
& E M G-E N F-F L G+E N F+F L G-E M G=0
\end{aligned}
$$

Ao adotar as curvaturas principais como linhas paramétricas é necessário que suas coordenadas sejam independentes, assim:

$$
\begin{aligned}
& \frac{d \theta}{d \xi}=0 \\
& \frac{d \xi}{d \theta}=0
\end{aligned}
$$

Logo:

$$
\lambda_{1}=0
$$

e, substituindo 2-35 na equação 2-27) resulta

$$
L F-M E=0 \quad M G-N F=0
$$

O próximo passo é demonstrar que $E G-F^{2}>0$. Pela equação $(2-5)$ :

$$
E G-F^{2}=\left(\hat{X}_{, \xi} \cdot \hat{X}_{, \xi}\right) \cdot\left(\hat{X}_{, \theta} \cdot \hat{X}_{, \theta}\right)-\left(\hat{X}_{, \xi} \cdot \hat{X}_{, \theta}\right)^{2}
$$

Utilizando a notação de módulo de uma derivada - retirando a vírgula do subíndice - , temos que $\hat{X}_{, \xi} \cdot \hat{X}_{, \xi}=\hat{X}_{\xi}$. Assim, pode-se definir o produto escalar entre $\hat{X}_{, \xi}$ e $\hat{X}_{, \theta}$ :

$$
\hat{X}_{, \xi} \cdot \hat{X}_{, \theta}=\hat{X}_{\xi} \hat{X}_{\theta} \cos \phi
$$

$\mathrm{Ou}$

$$
E G-F^{2}=\left(\hat{X}_{\xi} \hat{X}_{\theta}\right)^{2}\left(1-\cos ^{2} \phi\right)
$$

ou ainda

$$
E G-F^{2}=\left(\hat{X}_{\xi} \hat{X}_{\theta}\right)^{2} \sin ^{2} \phi>0 \quad \text { se } \quad \phi \neq 0
$$

Assim, como as linhas de curvatura principais são ortogonais, $\operatorname{com} F=0$, conforme a na equação $(2-8)$, e então $E G>0$. E, portanto, das equações $2-36$ conclui-se que:

$$
F=M=0
$$

Substituindo 2-37) na equação 2-20 obtem-se as expressões das curvaturas principais, como nessas curvas de coordenadas constantes as derivadas 
serão nulas i.e., $d \theta=0$ para a primeira curvatura e $d \xi=0$ para a segunda. Assim:

$$
\begin{aligned}
& K_{1}=\frac{1}{R_{1}}=\frac{L}{E} \\
& K_{2}=\frac{1}{R_{2}}=\frac{N}{G}
\end{aligned}
$$

Geometricamente, a figura 2.2 abaixo ilustra as duas curvaturas principais para uma superfície no espaço.

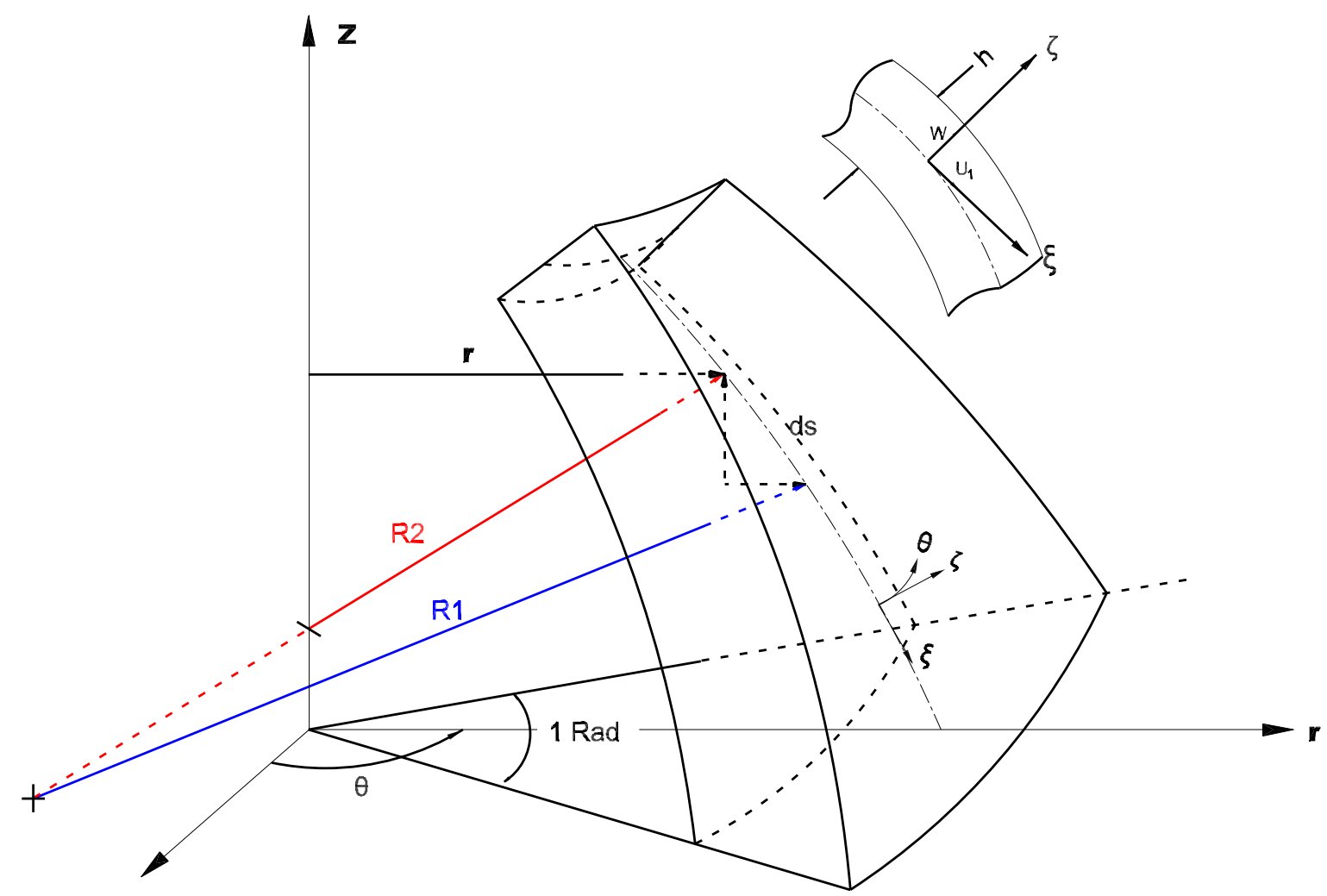

Figura 2.2: Curvaturas principais 


\subsection{3}

\section{Determinação dos vetores tangentes e normais das curvaturas principais}

Definindo os vetores unitários tangentes às curvaturas principais como

$$
\begin{gathered}
\hat{t}_{\xi}=\frac{\hat{X}_{, \xi}}{\left|\hat{X}_{\xi}\right|} \\
\hat{t}_{\theta}=\frac{\hat{X}_{, \theta}}{\left|\hat{X}_{\theta}\right|}
\end{gathered}
$$

utilizando o resultado dos coeficientes da equação 2-5 e considerando que os produtos internos serão iguais ao cosseno do ângulo igual a zero multiplicado ao quadrado do módulo do vetor. Assim,

$$
\begin{gathered}
\hat{t}_{\xi}=\frac{\hat{X}_{, \xi}}{\sqrt{E}} \\
\hat{t}_{\theta}=\frac{\hat{X}_{, \theta}}{\sqrt{G}}
\end{gathered}
$$

Susbstituindo pelos valores da equação $2-10$ tem-se

$$
\begin{gathered}
\hat{t}_{\xi}=\frac{\hat{X}_{, \xi}}{A_{1}} \\
\hat{t}_{\theta}=\frac{\hat{X}_{, \theta}}{A_{2}}
\end{gathered}
$$

O vetor unitário normal a superfície é resultado do produto vetorial entre os vetores unitários tangentes às curvaturas principais e, portanto:

$$
\hat{n}=\hat{t}_{\xi} \times \hat{t}_{\theta}=\frac{\hat{X}_{, \xi} \times \hat{X}_{, \theta}}{A_{1} A_{2}}
$$

A derivada do vetor normal em uma das coordenadas $\xi$ ou $\theta$ é perpendicular ao vetor normal, ou seja, pertencerá ao plano gerado por $\hat{t}_{\xi}$ e $\hat{t}_{\theta}$, assim pode ser escrito com o combinação linear dos vetores tangentes.

Tomando $\hat{n}_{, \xi}$ :

$$
\hat{n}_{, \xi}=a \hat{t}_{\xi}+b \hat{t}_{\theta}
$$

Da equação 2-40 e multiplicando pelo vetor $\hat{n}_{, \xi}$ :

$$
\begin{aligned}
& \hat{t}_{\xi} \cdot \hat{n}_{, \xi}=\frac{\hat{X}_{, \xi} \cdot \hat{n}_{, \xi}}{A_{1}}=a \hat{t}_{\xi} \cdot \hat{t}_{\xi}+b \hat{t}_{\theta} \cdot \hat{t}_{\xi}=a \\
& \hat{t}_{\theta} \cdot \hat{n}_{, \xi}=\frac{\hat{X}_{, \theta} \cdot \hat{n}_{, \xi}}{A_{2}}=a \hat{t}_{\xi} \cdot \hat{t}_{\theta}+b \hat{t}_{\theta} \cdot \hat{t}_{\theta}=b
\end{aligned}
$$


Substituindo os valores com os coeficientes encontrados na equação 2 221):

$$
\begin{gathered}
a=\frac{\hat{X}_{, \xi} \cdot \hat{n}_{, \xi}}{A_{1}}=\frac{L}{A_{1}} \\
b=\frac{\hat{X}_{, \theta} \cdot \hat{n}_{, \xi}}{A_{2}}=\frac{M}{A_{2}}=0
\end{gathered}
$$

Portanto, pela equação 2-43) e os coeficientes em 2-38) e (2-10) resulta

$$
\hat{n}_{, \xi}=\frac{L}{A_{1}} \hat{t}_{\xi}=\frac{E}{R_{1} A_{1}} \hat{t}_{\xi}=\frac{A_{1}}{R_{1}} \hat{t}_{\xi}
$$

Portanto:

$$
\hat{n}_{, \xi}=\frac{A_{1}}{R_{1}} \hat{t}_{\xi}
$$

Repetindo o processo acima, tem-se:

$$
\hat{n}_{, \theta}=\frac{A_{2}}{R_{2}} \hat{t}_{\theta}
$$




\section{2}

\section{Teoria de cascas}

Utilizando-se as equações básicas de Teoria da Elasticidade: equilíbrio, compatibilidade geométrica e constitutiva, o problema será modelado limitando-se para aplicação ao caso isotérmico, estático e de carregamento em cascas isotrópicas.

\subsection{1}

\section{Coordenadas de cascas}

Considera-se que os vetores normais durante a deformação da casca são preservados, implicando deslocamentos distribuidos linearmente ao longo da espessura da casca. Também, como referência utiliza-se a superfície média da casca. Um ponto interno da casca pode ser definido como o vetor $\hat{X}$ na superfície média mais uma coordenada $\zeta$ que varia de $\left[\frac{h}{2},-\frac{h}{2}\right]$, onde $h$ é espessura, na coordenada do vetor normal.

Portanto tem-se que

$$
\hat{R}(\xi, \theta, z)=\hat{X}(\xi, \theta)+\zeta \hat{n}(\xi, \theta)
$$

O comprimento do elemento infinitesimal é dado por:

$$
(d s)^{2}=d \hat{R} \cdot d \hat{R}=(d \hat{X}+\zeta d \hat{n}+d \zeta \hat{n}) \cdot(d \hat{X}+\zeta d \hat{n}+d \zeta \hat{n})
$$

Da equação acima, obtem-se

$$
(d s)^{2}=A_{1}^{2}\left(1+\frac{\zeta}{R_{1}}\right)^{2}(d \xi)^{2}+A_{2}^{2}\left(1+\frac{z}{R_{2}}\right)^{2}(d \theta)^{2}+(d \zeta)^{2}
$$

cujo desenvolvimento algébrico está apresentado no apêndice "Apêndice Fórmula 1" na página 85. 


\subsection{2}

\section{Deformações em coordenadas curvilíneas}

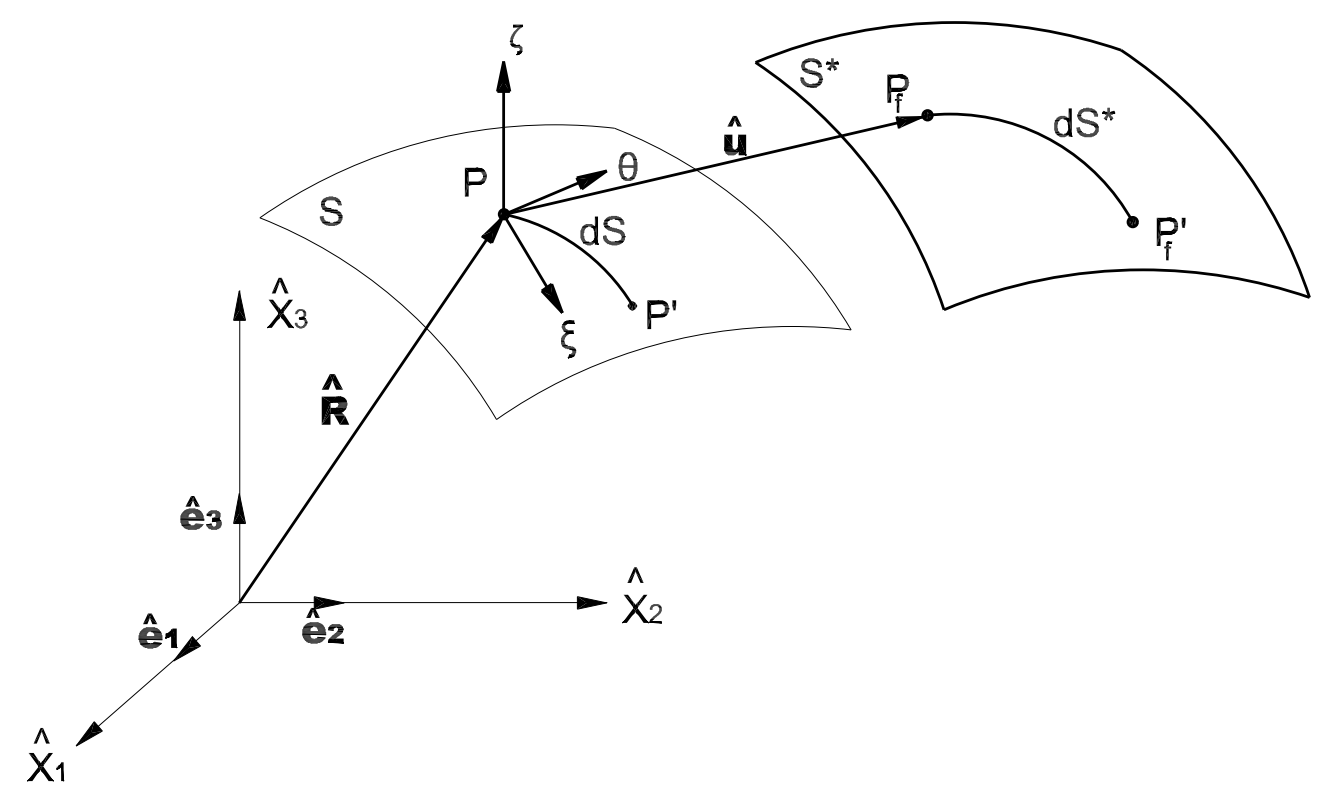

Figura 2.3: Casca com deformação

Em uma casca o vetor posição de um ponto $P$ é dado por: $\hat{R}(\xi, \theta, \zeta)$. Contudo para facilitar o desenvolvimento analítico as coordenadas serão reescritas e o vetor ficará: $\hat{R}\left(\alpha_{1}, \alpha_{2}, \alpha_{3}\right)$. Após o desenvolvimento completo este será retornado ao sistema de coordenadas padrão $\xi, \theta$ e $\zeta$.

O comprimento $(d s)^{2}$ da linha de um ponto $P$ a $P^{\prime}$ para um corpo indeformável é dado por:

$$
\begin{gathered}
(d s)^{2}=d \hat{R} \cdot d \hat{R}=\left(\frac{\partial \hat{R}}{\partial \alpha_{i}} \alpha_{i}\right) \cdot\left(\frac{\partial \hat{R}}{\partial \alpha_{j}} \alpha_{j}\right) \\
(d s)^{2}=\sum_{i=1}^{3}\left(\frac{\partial \hat{R}}{\partial \alpha_{i}} \cdot \frac{\partial \hat{R}}{\partial \alpha_{i}}\right)\left(d \alpha_{i}\right)^{2} \equiv \sum_{i=1}^{3} A_{i}^{2}\left(d \alpha_{i}\right)^{2}
\end{gathered}
$$

Para simplificar a equação acima pode-se utilizar o parâmetro de Lamé, definido po $A_{k}=A_{k}\left(\alpha_{1}, \alpha_{2}, \alpha_{3}\right)$. Como as coordenadas são ortogonais tem-se

$$
\left(\frac{\partial \hat{R}}{\partial \alpha_{i}}\right) \cdot\left(\frac{\partial \hat{R}}{\partial \alpha_{j}}\right)=0 \quad, i \neq j
$$

e, portanto:

$$
A_{i}^{2}=\frac{\partial \hat{R}}{\partial \alpha_{i}} \cdot \frac{\partial \hat{R}}{\partial \alpha_{i}} \equiv \hat{R}_{, i} \cdot \hat{R}_{, i}
$$

Assim, $A_{i}=\left|\hat{R}_{, i}\right|$, ou seja, a magnitue do vetor $\hat{R}_{, i}$ e o vetor tangente a 
$\alpha_{i}$ pode ser facilmente definido:

$$
\hat{t}_{\alpha_{i}}=\frac{\hat{R}_{, i}}{\left|\hat{R}_{, i}\right|}=\frac{\hat{R}_{, i}}{A_{i}}
$$

O vetor deslocamento do ponto $P^{\prime}$, dado por $\hat{u}=\left(u_{1}, u_{2}, u_{3}\right)$ é reescrito utilizando os vetores tangentes. Assim:

$$
\hat{u}=\frac{u_{1}}{A_{1}} \hat{R}_{, 1}+\frac{u_{2}}{A_{2}} \hat{R}_{, 2}+\frac{u_{3}}{A_{3}} \hat{R}_{, 3}=\sum_{k=1}^{3} \frac{u_{k}}{A_{k}} \hat{R}_{, k}
$$

Agora, conforme ilustrado na figura 2.3. considera-se a deformação do corpo. Nos pontos $P$ e $P^{\prime}$ do corpo indeformáveis, com coordenadas $\alpha_{i}$ e $\alpha_{i}+d \alpha_{i}$ irão, com a deformação, para os pontos $P_{f}$ e $P_{f}^{\prime}$ que apresentam coordenadas $\alpha_{i}+\frac{u_{i}}{A_{i}}$ e $\alpha_{i}+\frac{u_{i}}{A_{i}}+d \alpha_{i}+d\left(\frac{u_{i}}{A_{i}}\right)$.

Pode-se definir:

$$
\eta_{i}=\frac{u_{i}}{A_{i}}
$$

Por conveniência, pode-se tabular as coordenadas dependentes dos pontos modelados, assim:

$$
\begin{gathered}
P\left(\alpha_{1}, \alpha_{2}, \alpha_{3}\right) \\
P^{\prime}\left(\alpha_{1}+d \alpha_{1}, \alpha_{2}+d \alpha_{2}, \alpha_{3}+d \alpha_{3}\right) \\
P_{f}\left(\alpha_{1}+\eta_{1}, \alpha_{2}+\eta_{2}, \alpha_{3}+\eta_{3}\right) \\
P_{f}^{\prime}\left(\alpha_{1}+d \alpha_{1}+\eta_{1}, \alpha_{2}+d \alpha_{2}+\eta_{2}, \alpha_{3}+d \alpha_{3}+\eta_{3}\right)
\end{gathered}
$$

O parâmetro de Lamé para o ponto $P_{f}$ pode ser aproximado, portanto:

$$
A_{i}^{2}\left(\alpha_{1}+\eta_{1}, \alpha_{2}+\eta_{2}, \alpha_{3}+\eta_{3}\right) \approx A_{i}^{2}\left(\alpha_{1}, \alpha_{2}, \alpha_{3}\right)+\sum_{j=1}^{3} \frac{\partial A_{i}^{2}}{\partial \alpha_{j}} \eta_{j}
$$

O comprimento $\left(d s^{*}\right)^{2}$ é dado por:

$$
\left(d s^{*}\right)^{2}=\sum_{i=1}^{3}\left[A_{i}^{2}\left(\alpha_{1}+\eta_{1}, \alpha_{2}+\eta_{2}, \alpha_{3}+\eta_{3}\right) \cdot\left(d \alpha_{i}+\sum_{j=1}^{3} \frac{\partial \eta_{i}}{\partial \alpha_{j}} d \alpha_{j}\right)^{2}\right]
$$

Substituindo a equação (2-54) na equação (2-55) acima, tem-se:

$$
\left(d s^{*}\right)^{2}=\sum_{i=1}^{3}\left[\left(A_{i}^{2}\left(\alpha_{1}, \alpha_{2}, \alpha_{3}\right)+\sum_{j=1}^{3} \frac{\partial A_{i}^{2}}{\partial \alpha_{j}} \eta_{j}\right) \cdot\left(d \alpha_{i}+\sum_{j=1}^{3} \frac{\partial \eta_{i}}{\partial \alpha_{j}} d \alpha_{j}\right)^{2}\right]
$$

$\mathrm{Ou}$ 
$\left(d s^{*}\right)^{2}=\sum_{i=1}^{3} A_{i}^{2}\left(\alpha_{1}, \alpha_{2}, \alpha_{3}\right)+\sum_{i=1}^{3} \sum_{j=1}^{3}\left[\frac{\partial A_{i}^{2}}{\partial \alpha_{j}} \eta_{j}\left(d \alpha_{i}\right)^{2}+A_{i}^{2} \frac{\partial \eta_{i}}{\partial \alpha_{j}} d \alpha_{j} d \alpha_{i}+A_{j}^{2} \frac{\partial \eta_{j}}{\partial \alpha_{i}} d \alpha_{i} d \alpha_{j}\right]$

Combinando as duas equações $2-56)$ e $2-50)$, pode-se calcular $\left(d s^{*}\right)^{2}-$ $(d s)^{2}$ obtem-se

$$
\left(d s^{*}\right)^{2}-(d s)^{2}=\sum_{i=1}^{3} \sum_{j=1}^{3}\left[\frac{\partial A_{i}^{2}}{\partial \alpha_{j}} \eta_{j}\left(d \alpha_{i}\right)^{2}+\left(A_{i}^{2} \frac{\partial \eta_{i}}{\partial \alpha_{j}}+A_{j}^{2} \frac{\partial \eta_{j}}{\partial \alpha_{i}}\right)\left(d \alpha_{i} d \alpha_{j}\right)\right]
$$

Utilizando o delta de Kronecker $\operatorname{com} \delta_{i j}=1$ quando $i=j$ e $\delta_{i j}=0$ quando $i \neq j$ tem-se

$$
\left(d s^{*}\right)^{2}-(d s)^{2}=\sum_{i=1}^{3} \sum_{j=1}^{3}\left[\sum_{k=1}^{3} \frac{\partial A_{i}^{2}}{\partial \alpha_{k}} \eta_{k}\left(d \alpha_{i} d \alpha_{j}\right) \delta_{i j}+\left(A_{i}^{2} \frac{\partial \eta_{i}}{\partial \alpha_{j}}+A_{j}^{2} \frac{\partial \eta_{j}}{\partial \alpha_{i}}\right)\left(d \alpha_{i} d \alpha_{j}\right)\right]
$$

$\mathrm{Ou}$

$$
\left(d s^{*}\right)^{2}-(d s)^{2}=\sum_{i=1}^{3} \sum_{j=1}^{3}\left[\sum_{k=1}^{3} \frac{\partial A_{i}^{2}}{\partial \alpha_{k}} \eta_{k} \delta_{i j}+A_{i}^{2} \frac{\partial \eta_{i}}{\partial \alpha_{j}}+A_{j}^{2} \frac{\partial \eta_{j}}{\partial \alpha_{i}}\right]\left(d \alpha_{i} d \alpha_{j}\right)
$$

então

$$
\frac{\left(d s^{*}\right)^{2}-(d s)^{2}}{(d s)^{2}}=\sum_{i=1}^{3} \sum_{j=1}^{3}\left[\sum_{k=1}^{3} \frac{\partial A_{i}^{2}}{\partial \alpha_{k}} \eta_{k} \delta_{i j}+A_{i}^{2} \frac{\partial \eta_{i}}{\partial \alpha_{j}}+A_{j}^{2} \frac{\partial \eta_{j}}{\partial \alpha_{i}}\right]\left(\frac{d \alpha_{i}}{d s}\right)\left(\frac{d \alpha_{j}}{d s}\right)
$$

A definição da deformação de um elemento linear é dado por:

$$
\epsilon=\frac{\left(d s^{*}\right)^{2}-(d s)^{2}}{(d s)^{2}}
$$

Além disso, o tensor das deformações $\left(\epsilon_{i j}\right)$ é dado por:

$$
\left(d s^{*}\right)^{2}-(d s)^{2}=\sum_{i=1}^{3} \sum_{j=1}^{3} 2 \epsilon_{i j} l_{i} l_{j}(d s)^{2}
$$

onde $l_{i}$ são os cossenos diretores do elemento $d s$ com respeito a $d \alpha_{i}$. Cosseno diretor é o resultado do cosseno fruto da divisão do produto interno entre dois vetores e seus módulos. $l_{k}$ é definido como:

$$
l_{k}=A_{k} \frac{d \alpha_{k}}{d s}
$$

A equação 2-59) pode ser reescrita como: 


$$
\frac{d s^{*}}{d s}=1+\epsilon
$$

Assim:

$$
\left(d s^{*}\right)^{2}-(d s)^{2}=\left(\frac{d s^{*}}{d s}\right)^{2}(d s)^{2}-(d s)^{2}=\left[(1+\epsilon)^{2}-1\right](d s)^{2}=\left(\epsilon^{2}+2 \epsilon\right)(d s)^{2}
$$

Sabendo que os valores numéricos de deformação são muito baixos podese considerar, em primeira aproximação, $\epsilon \ll 1$, e assim desconsiderar os termos quadráticos da expressão. Portanto:

$$
\left(d s^{*}\right)^{2}-(d s)^{2}=2 \epsilon(d s)^{2}
$$

que substituindo em $(2-60)$, resulta

$$
2 \epsilon(d s)^{2}=\sum_{i=1}^{3} \sum_{j=1}^{3} 2 \epsilon_{i j} l_{i} l_{j}(d s)^{2}
$$

Logo, as componenstes do tensor das deformações podem ser escritos

$$
\epsilon=\sum_{i=1}^{3} \sum_{j=1}^{3} \epsilon_{i j} l_{i} l_{j}
$$

Substituindo $2-63)$ em $2-57$ resulta

$$
2 \epsilon(d s)^{2}=\sum_{i=1}^{3} \sum_{j=1}^{3}\left[\sum_{k=1}^{3} \frac{\partial A_{i}^{2}}{\partial \alpha_{k}} \eta_{k} \delta_{i j}+A_{i}^{2} \frac{\partial \eta_{i}}{\partial \alpha_{j}}+A_{j}^{2} \frac{\partial \eta_{j}}{\partial \alpha_{i}}\right]\left(d \alpha_{i} d \alpha_{j}\right)
$$

e substituindo 2-64 em 2-65 tem-se

$$
2 \sum_{i=1}^{3} \sum_{j=1}^{3} \epsilon_{i j} l_{i} l_{j}(d s)^{2}=\sum_{i=1}^{3} \sum_{j=1}^{3}\left[\sum_{k=1}^{3} \frac{\partial A_{i}^{2}}{\partial \alpha_{k}} \eta_{k} \delta_{i j}+A_{i}^{2} \frac{\partial \eta_{i}}{\partial \alpha_{j}}+A_{j}^{2} \frac{\partial \eta_{j}}{\partial \alpha_{i}}\right]\left(d \alpha_{i} d \alpha_{j}\right)
$$

Susbtituindo-se (2-61) em 2-66 temos

$2 \sum_{i=1}^{3} \sum_{j=1}^{3} \epsilon_{i j} l_{i} l_{j}(d s)^{2}=\sum_{i=1}^{3} \sum_{j=1}^{3}\left[\sum_{k=1}^{3} \frac{\partial A_{i}^{2}}{\partial \alpha_{k}} \eta_{k} \delta_{i j}+A_{i}^{2} \frac{\partial \eta_{i}}{\partial \alpha_{j}}+A_{j}^{2} \frac{\partial \eta_{j}}{\partial \alpha_{i}}\right]\left(\frac{l_{i}(d s)}{A_{i}}\right)\left(\frac{l_{i}(d s)}{A_{i}}\right)$

que, simplificando, resulta

$$
2 \epsilon_{i j}=\frac{1}{A_{i} A_{j}}\left[\sum_{k=1}^{3} \frac{\partial A_{i}^{2}}{\partial \alpha_{k}} \eta_{k} \delta_{i j}+A_{i}^{2} \frac{\partial \eta_{i}}{\partial \alpha_{j}}+A_{j}^{2} \frac{\partial \eta_{j}}{\partial \alpha_{i}}\right]
$$

Para tensões normais tem-se que $i=j$, logo: 


$$
\epsilon_{i j}=\frac{\partial}{\partial \alpha_{i}}\left(\frac{U_{i}}{A_{i}}\right)+\frac{1}{2 A_{i}^{2}} \sum_{k=1}^{3} \frac{\partial A_{i}}{\partial \alpha_{k}}\left(\frac{U_{k}}{A_{k}}\right)
$$

Para a tensão cisalhante $\gamma_{i j}=2 \epsilon_{i j}$ quando $i \neq j$ :

$$
\gamma_{i j}=\frac{A_{i}}{A_{j}} \frac{\partial}{\partial \alpha_{j}}\left(\frac{U_{i}}{A_{i}}\right)+\frac{A_{j}}{A_{i}} \frac{\partial}{\partial \alpha_{i}}\left(\frac{U_{j}}{A_{j}}\right)
$$

Portanto:

$$
\begin{gathered}
\epsilon_{\xi \xi}=\frac{1}{A_{1}\left(1+\frac{T}{R_{1}}\right)}\left(\frac{\partial U_{1}}{\partial \xi}+\frac{U_{2}}{A_{2}} \frac{\partial A_{1}}{\partial \theta}+\frac{A_{1} W}{R_{1}}\right) \\
\epsilon_{\theta \theta}=\frac{1}{A_{2}\left(1+\frac{T}{R_{2}}\right)}\left(\frac{\partial U_{2}}{\partial \theta}+\frac{U_{1}}{A_{1}} \frac{\partial A_{2}}{\partial \xi}+\frac{A_{2} W}{R_{2}}\right) \\
\epsilon_{\zeta \zeta}=\frac{\partial W}{\partial T} \\
\gamma_{\xi \theta}=\frac{A_{2}\left(1+\frac{T}{R_{2}}\right)}{A_{1}\left(1+\frac{T}{R_{1}}\right)} \frac{\partial}{\partial \xi}\left[\frac{U_{2}}{A_{2}\left(1+\frac{T}{R_{2}}\right)}\right]+\frac{A_{1}\left(1+\frac{T}{R_{1}}\right)}{A_{2}\left(1+\frac{T}{R_{2}}\right)} \frac{\partial}{\partial \theta}\left[\frac{U_{1}}{A_{1}\left(1+\frac{T}{R_{1}}\right)}\right] \\
\gamma_{\xi \zeta}=\frac{1}{A_{1}\left(1+\frac{T}{R_{1}}\right)} \frac{\partial W}{\partial \xi}+A_{1}\left(1+\frac{T}{R_{1}}\right) \frac{\partial}{\partial T}\left[\frac{U_{1}}{A_{1}\left(1+\frac{T}{R_{1}}\right)}\right] \\
\gamma_{\theta \zeta}=\frac{1}{A_{2}\left(1+\frac{T}{R_{2}}\right)} \frac{\partial W}{\partial \xi}+A_{2}\left(1+\frac{T}{R_{2}}\right) \frac{\partial}{\partial T}\left[\frac{U_{1}}{A_{2}\left(1+\frac{T}{R_{2}}\right)}\right]
\end{gathered}
$$

onde:

$$
A_{1}=\frac{d s}{d \xi} \quad A_{2}=r \quad T=\frac{h}{2} \zeta
$$

Na figura 2.4 abaixo pode-se visualizar a localização de $r$ :

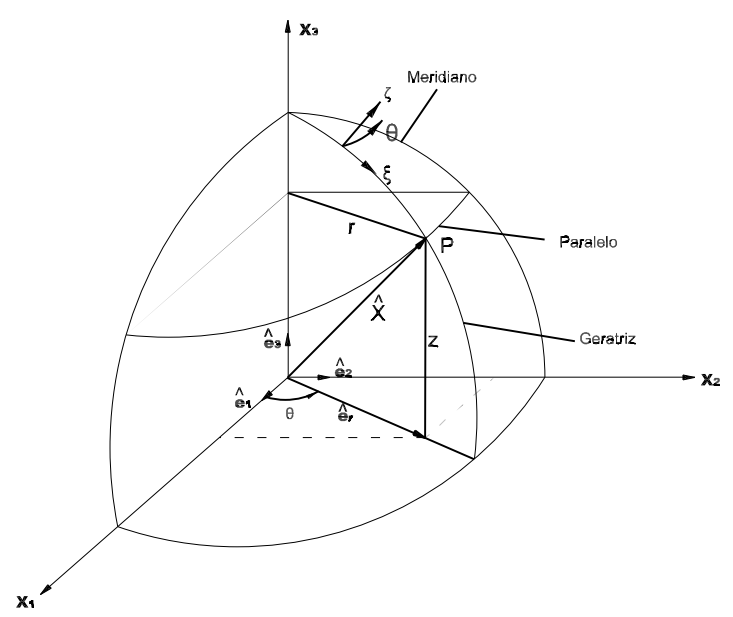

Figura 2.4: Casca 


\subsection{3}

\section{Simplificação para Cascas Cilíndricas}

O desenvolvimento de cascas pode ser reduzido para o caso de cascas cilíndricas axissimétricas. Para isso, a primeira consequência devido a aximetria é a anulação do deslocamento circunferencial, $U_{2}$. Portanto:

$$
U_{2}=0
$$

A segunda característica que deve ser apresentada é o raio principal de curvatura. Ao se adaptar uma esfera em cilindro o primeiro raio de curvatura tenderá a infinito, enquanto o segundo será o raio do cilindro, assim:

$$
\begin{gathered}
R_{1} \rightarrow \infty \\
R_{2}=R
\end{gathered}
$$

Portanto, impondo-se estas restrições nas equações das deformações estas medidas podem ser reescritas na forma:

$$
\begin{gathered}
\epsilon_{\xi \xi}=\frac{1}{s^{\prime}}\left(\frac{\partial U_{1}}{\partial \xi}\right) \\
\epsilon_{\theta \theta}=\frac{W}{R_{2}+T} \\
\epsilon_{\zeta \zeta}=\frac{\partial W}{\partial T} \\
\gamma_{\xi \zeta}=\frac{1}{s^{\prime}} \frac{\partial W}{\partial \xi}+\frac{\partial U_{1}}{\partial T} \\
\gamma_{\xi \theta}=\gamma_{\theta \zeta}=0
\end{gathered}
$$




\section{Desenvolvimento do Método dos Elementos Finitos}

\section{1}

\section{Introdução}

O método dos elementos finitos é um procedimento numérico de discretização objetivando determinar os deslocamentos de um corpo garantidas as condições de equilíbrio e de contorno. Este consiste na discretização de um funcional $\Pi$ correspondente ao Princípio dos Deslocamentos Virtuais, enunciado na forma:

"O equilíbrio de um corpo é satisfeito para qualquer campo admissível (compatível) de deslocamentos sempre que o trabalho virtual interno for igual ao trabalho virtual externo."

Evitando-se longos desenvolvimentos, o método resulta na seguinte proposição.

$$
\begin{aligned}
& {\left[\sum_{m=1}^{M} \int_{V^{(m)}}\left(\underline{\left.B^{(m)}\right)_{(x, y, z)}^{T}} \underline{C^{(m)}}\left(\underline{B^{(m)}}\right)_{(x, y, z)}^{T} d V^{(m)}\right] \widehat{\widehat{u}}=\right.} \\
& \sum_{m=1}^{M} \int_{V^{(m)}}\left(\underline{H^{(m)}}\right)^{T} \underline{f^{B}} d V^{(m)}+\sum_{m=1}^{M} \int_{S^{(m)}}\left(\underline{H^{(m)}}\right)^{T} \underline{f^{S}} d S^{(m)}+\sum_{i} \underline{R_{c}^{i}} \\
& \quad-\sum_{m=1}^{M} \int_{V^{(m)}}\left(\underline{B^{(m)}}\right)_{(x, y, z)}^{T}\left(\underline{\tau_{I}}\right)_{(x, y, z)}^{(m)} d V^{(m)}
\end{aligned}
$$

Onde:

$-(\underline{K})^{(m)}=\int_{V^{(m)}}\left(\underline{B^{(m)}}\right)_{(x, y, z)}^{T} \underline{C^{(m)}}\left(\underline{B^{(m)}}\right)_{(x, y, z)}^{T} d V^{(m)}$ é a matriz de Rigidez elementar

- $\left(\underline{R_{B}}\right)^{(m)}=\int_{V^{(m)}}\left(\underline{H^{(m)}}\right)^{T} \underline{f^{B}} d V^{(m)}$ é o vetor com as forças de corpo elementar

- $\left(\underline{R_{S}}\right)^{(m)}=\int_{S^{(m)}}\left(\underline{H^{(m)}}\right)^{T} \underline{f^{S}} d S^{(m)}$ é o vetor com as forças de superfície elementar

$-\left(\underline{R_{I}}\right)^{(m)}=\int_{V^{(m)}}\left(\underline{B^{(m)}}\right)_{(x, y, z)}^{T}\left(\underline{\tau_{I}}\right)_{(x, y, z)}^{(m)} d V^{(m)}$ é o vetor dos carregamentos iniciais.

A discretização do domínio, representativo de um elemento $m$, região do corpo, revela-se:

$$
\underline{u^{(m)}}=\underline{H^{(m)}}(x, y, z) \underline{\widehat{u}}
$$


Onde o vetor $\underline{\widehat{u}}$ apresenta o os deslocamentos globais do sistema e a matriz $\underline{H^{(m)}}{ }_{(x, y, z)}$ trata-se da interpolação dos deslocamentos utilizando uma função de Lagrange.

A equação de Compatibilidade Geométrica é dada para um elemento m,

$$
\underline{\epsilon^{(m)}}=\underline{B^{(m)}}(x, y, z) \underline{\widehat{u}}
$$

Onde a matriz $\underline{B^{(m)}}(x, y, z)$ é obtida pela aproximação diferencial e combinação das linhas da matriz $\underline{H^{(m)}}(x, y, z)$.

Finalmente, a equação constitutiva para um elemento m é dada por::

$$
\underline{\tau^{(m)}}=\underline{\tau_{I}^{(m)}}+\underline{C^{(m)}} \underline{B^{(m)}}(x, y, z) \underline{\widehat{u}}
$$

$\tau_{I}^{(m)}$ é o vetor da em um elemento m, a matriz $\underline{C^{(m)}}$ é a matriz de elasticidade para o elemento $\mathrm{m}$ e o vetor $\underline{\tau^{(m)}}$ é a tensão no elemento finito $\mathrm{m}$.

Para problemas estáticos o seguinte sistema de equações fica assim definido

$$
\underline{K \widehat{u}}=\underline{R_{B}}+\underline{R_{C}}+\underline{R_{S}}-\underline{R_{I}}
$$

para o vetor deslocamento $\underline{\widehat{u}}$.

Enquanto que, para problemas dinâmicos, a força de corpo, definida pelo vetor $\underline{f^{B}}$ é modificada, para

$$
\left(f^{B}\right)^{(m)}-\rho^{(m)}(\underline{\ddot{u}})^{(m)}
$$

e, portanto, resulta

$$
\sum_{m=1}^{M} \int_{V^{(m)}}\left(\underline{H^{(m)}}\right)^{T} \underline{\rho^{(m)}} \underline{H^{(m)}} d V^{(m)} \underline{\ddot{u}}+\underline{K \widehat{u}}=\underline{R}
$$

Onde a Matriz M, de massa global, é definida por:

$$
\underline{M}=\sum_{m=1}^{M} \int_{V^{(m)}}\left(\underline{H^{(m)}}\right)^{T} \underline{\rho^{(m)}} \underline{H^{(m)}} d V^{(m)}
$$

\section{2}

\section{Funções de deslocamento}

Na solução numérica do problema de vasos de pressão utiliza-se o método dos elementos finitos com discretização na direção axial do vaso, enquanto na direção radial utiliza-se uma função resultante da combinação de soluções analíticas.

Da solução da equação diferencial refrente ao deslocamento radial, resul- 
tante da solução de Lamé, equação (1-11) fornece para cascas cilíndricas.

$$
W_{\text {Lamé }}=A_{1} r+\frac{A_{2}}{r}
$$

enquanto o deslocamento radial para cascas esféricas, tem-se :

$$
W_{\text {Esfera }}=A_{3} r+\frac{A_{4}}{r^{2}}
$$

e o deslocamento radial para cilindros com rotação:

$$
W_{\text {Rotação }}=A_{5} r+\frac{A_{6}}{r}+A_{7} r^{3}
$$

Combinando-se as três soluções acima resulta:

$$
W(\xi, \zeta)=W_{0}(\xi)+\frac{W_{1}(\xi)}{R(\xi, \zeta)}+W_{2}(\xi) R(\xi, \zeta)+\frac{W_{3}(\xi)}{[R(\xi, \zeta)]^{2}}+W_{4}(\xi, \zeta)[R(\xi, \zeta)]^{3}
$$

onde a coordenada radial

$$
R(\xi, \zeta)=R_{2}(\xi)+T(\zeta)
$$

com $T(\zeta)$ sendo a coordenada referida ao ponto médio da espessura do vaso de pressão,com a coordenada $\zeta$ variando de $[-1,1]$. Assim:

$$
T(\zeta)=\frac{h}{2} \zeta
$$

enquanto para o deslocamento axial utiliza-se uma função interpoladora de terceiro grau, com coordenada definida por $T(\zeta)$.

$$
U_{1}(\xi, \zeta)=U_{1}^{0}(\xi)+\gamma_{1}(\xi) T+\Phi_{1}(\xi) T^{2}+\Psi_{1}(\xi) T^{3}
$$

\section{3}

\section{Condições de contorno naturais}

No modelo considerado a condição de contorno natural deve ser garantida, sem nenhuma restrição adicionale nesta condição as tensões de cisalhamento nas faces internas e externas da casca são necessariamente nulas, que correspondem a deformação angular $\gamma_{\xi \zeta}=0$ nas faces da casca. Assim, tem-se que

$$
\left.\gamma_{\xi \zeta}\right|_{\zeta= \pm 1}=0
$$

Onde, pela equação (2-80), resulta em

$$
\gamma_{\xi \zeta}=\frac{1}{s^{\prime}} \cdot \frac{\partial W}{\partial \xi}+\frac{\partial U_{1}}{\partial T}
$$


Desenvolvendo-se as duas equações acima, obtem-se

$$
\begin{gathered}
\phi_{1}(\xi)=-\left(\left.\frac{1}{s^{\prime}} \frac{\partial W}{\partial \xi}\right|_{\zeta=+1}-\left.\frac{1}{s^{\prime}} \frac{\partial W}{\partial \xi}\right|_{\zeta=-1}\right) \cdot \frac{1}{2 h} \\
\psi_{1}(\xi)=-\left(\left.\frac{1}{s^{\prime}} \frac{\partial W}{\partial \xi}\right|_{\zeta=+1}+\left.\frac{1}{s^{\prime}} \frac{\partial W}{\partial \xi}\right|_{\zeta=-1}\right) \cdot \frac{2}{3 h^{2} s^{\prime}}-\frac{4 \gamma_{1}}{3 h^{2}}
\end{gathered}
$$

Um aspecto importante é a redução no número de graus de liberdade por nó do elemento. A quantidade de constantes presentes na função de deslocamento radial mostrado na equação (3-9) adicionada ao do na equação deslocamento axial (3-12) totaliza 9 que, contudo, não são independentes, conforme equações (3-15) e (3-16), sendo reescritos em funções dos 7 graus de liberdade em cada nó.

O desenvolvimento completo das equações $(3-15)$ e (3-16) está apresentado no apêndice da página 87.

\section{4}

\section{Funções lagrangeanas}

Considerando-se o deslocamento axial, funções lagrangeanas são utilizadas para interpolar os graus de liberdade na direção longitudinal da casca. São construidas para um intervalo padrão de $[-1,1]$ de coordenadas que são transformados para as coordenadas geométricas do elemento através da transformação jacobiana. Em termos de modelagem numérica esta transformação facilitará a integração numérica das equações, que no presente estado empregue a quadratura de Gauss. 


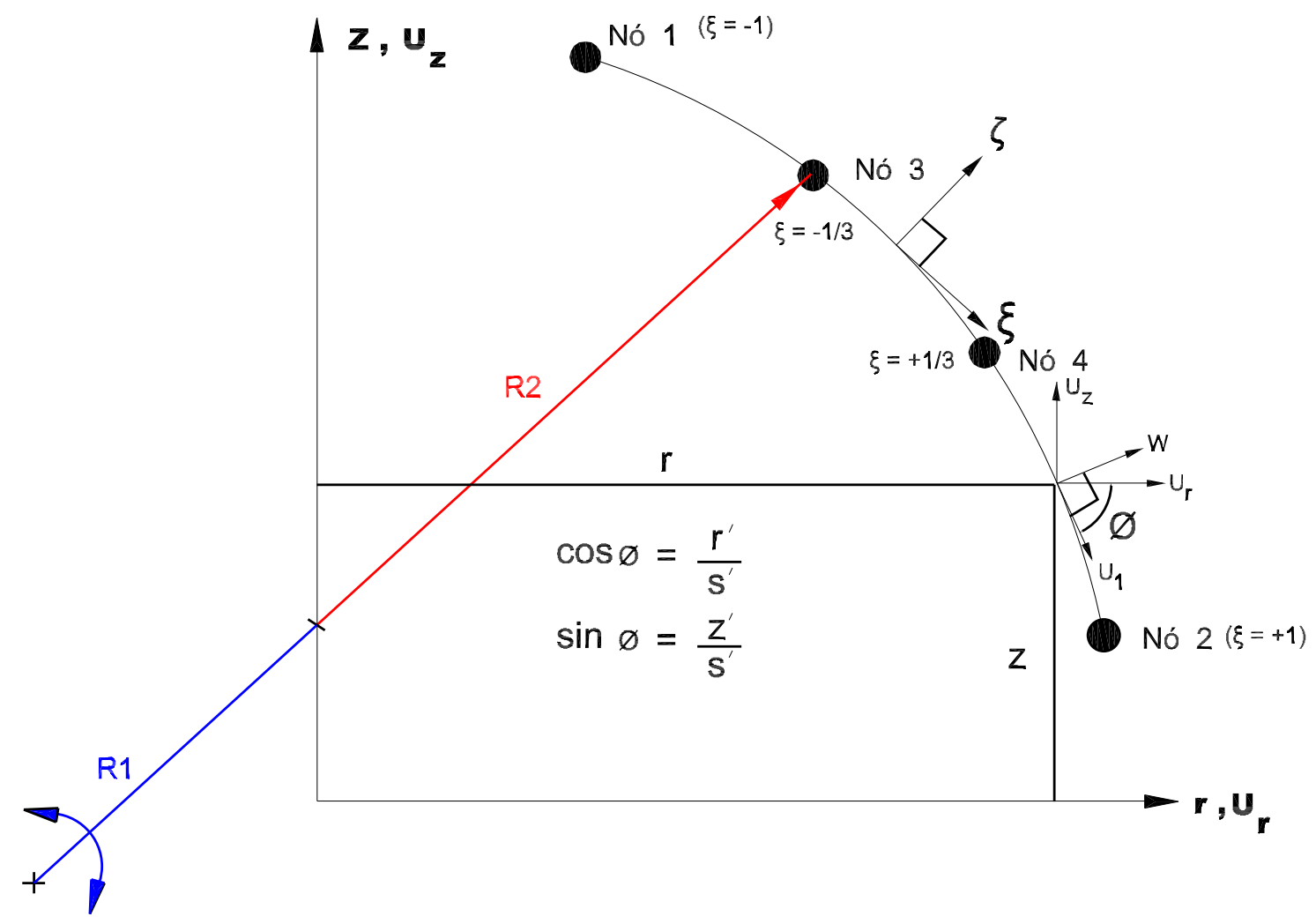

Figura 3.1: Disposição dos nós no elemento

Considerando-se o elemento de quatro nós montrado na figura 3.1, tem-se as seguintes funções de interpolação

$$
\begin{gathered}
h_{1}(\xi)=\frac{-9 \xi^{3}+9 \xi^{2}+\xi-1}{16} \\
h_{2}(\xi)=\frac{9 \xi^{3}+9 \xi^{2}-\xi-1}{16} \\
h_{3}(\xi)=\frac{27 \xi^{3}-9 \xi^{2}-27 \xi+9}{16} \\
h_{4}(\xi)=\frac{-27 \xi^{3}-9 \xi^{2}+27 \xi+9}{16}
\end{gathered}
$$

que permitem interpolar no domínio do elemento uma grandeza por uma função contínua conhecidos seus valores em pontos discretos. Desta forma, tem-se, por exemplo:

o raio variando na coordenada $\xi$ :

$$
r(\xi)=\sum_{i=1}^{k} h_{i}(\xi) r_{i}
$$

o comprimento longitudinal $z$ do vaso de pressão com a variação de $\xi$ :

$$
z(\xi)=\sum_{i=1}^{k} h_{i}(\xi) z_{i}
$$


as funções de deslocamentos, expressas nas equações $(3-9)$ e $(3-12)$, cujas contantes variam com a coordenada $\xi$, e são reescritas da contribuição de cada nó, para determinar-se a matriz $\underline{H^{(m)}}$. Desta forma, utilizando-se as funções lagrangeanas, tem-se:

$$
\begin{aligned}
& W_{0}(\xi)=\sum_{i=1}^{k} h_{i}(\xi) W_{0}^{i} \\
& W_{1}(\xi)=\sum_{i=1}^{k} h_{i}(\xi) W_{1}^{i} \\
& W_{2}(\xi)=\sum_{i=1}^{k} h_{i}(\xi) W_{2}^{i} \\
& W_{3}(\xi)=\sum_{i=1}^{k} h_{i}(\xi) W_{3}^{i} \\
& W_{4}(\xi)=\sum_{i=1}^{k} h_{i}(\xi) W_{4}^{i}
\end{aligned}
$$




\section{5}

\section{Vetor $\hat{u}$}

O vetor deslocamento $\underline{\underline{u}}$ é definido usando-se a distribuição dos nós conforme está ilustrado na figura 3.1. A ordem seguida dos graus de liberdade é dada por: $U_{0}, \gamma_{1}, W_{0}, W_{1}, W_{2}, W_{3}$ e $W_{4}$. Desta forma, considerando os quatro nós do elemento tem-se

$$
\left.[\underline{\underline{u}}]=\quad \begin{array}{ccccccc}
U_{0}^{1} & \gamma_{1}^{1} & W_{0}^{1} & W_{1}^{1} & W_{2}^{1} & W_{3}^{1} & W_{4}^{1} \\
U_{0}^{3} & \gamma_{1}^{3} & W_{0}^{3} & W_{1}^{3} & W_{2}^{3} & W_{3}^{3} & W_{4}^{3} \\
U_{0}^{4} & \gamma_{1}^{4} & W_{0}^{4} & W_{1}^{4} & W_{2}^{4} & W_{3}^{4} & W_{4}^{4} \\
U_{0}^{2} & \gamma_{1}^{2} & W_{0}^{2} & W_{1}^{2} & W_{2}^{2} & W_{3}^{2} & W_{4}^{2}
\end{array}\right]
$$

\section{6}

Matriz $H^{(m)}$

A matriz $\underline{H^{(m)}}$ relaciona os deslocamentos naturais dos graus de liberdade do problema. Observando-se a discretização do funcional $\Pi$ de elementos finitos a matriz $\underline{H^{(m)}}$ é utilizada para interpolar os carregamentos. Levando em consideração que os carregamentos modelados encontram-se no sentido radial, a equação (3-2) resulta somente para deslocamentos radiais.

Será definido, portanto o vetor hessiana que só terá componete radial, denotado como $\underline{H_{W}^{(m)}}$.

Logo:

$$
\begin{aligned}
& {\left[0 \quad 0 \quad h_{1}(\xi) \quad \frac{h_{1}(\xi)}{R(\xi, \zeta} \quad h_{1}(\xi) \cdot R(\xi, \zeta) \quad \frac{h_{1}(\xi)}{R(\xi, \zeta)^{2}} \quad h_{1}(\xi) \cdot R(\xi, \zeta)^{3}\right.} \\
& {\left[\underline{H_{W}^{(m)}}\right]=\quad \begin{array}{llllllll}
0 & 0 & h_{3}(\xi) & \frac{h_{3}(\xi)}{R(\xi, \zeta} & h_{3}(\xi) \cdot R(\xi, \zeta) & \frac{h_{3}(\xi)}{R(\xi, \zeta)^{2}} & h_{3}(\xi) \cdot R(\xi, \zeta)^{3} \\
\quad 0 & 0 & h_{4}(\xi) & \frac{h_{4}(\xi)}{R(\xi, \zeta} & h_{4}(\xi) \cdot R(\xi, \zeta) & \frac{h_{4}(\xi)}{R(\xi, \zeta)^{2}} & h_{4}(\xi) \cdot R(\xi, \zeta)^{3}
\end{array}} \\
& \left.\begin{array}{lllllll}
0 & 0 & h_{2}(\xi) & \frac{h_{2}(\xi)}{R(\xi, \zeta} & h_{2}(\xi) \cdot R(\xi, \zeta) & \frac{h_{2}(\xi)}{R(\xi, \zeta)^{2}} & h_{2}(\xi) \cdot R(\xi, \zeta)^{3}
\end{array}\right]
\end{aligned}
$$

A fim de facilitar a visualização, o deslocamento radial elementar pode ser descrito em função de 28 graus de liberdades, como: 


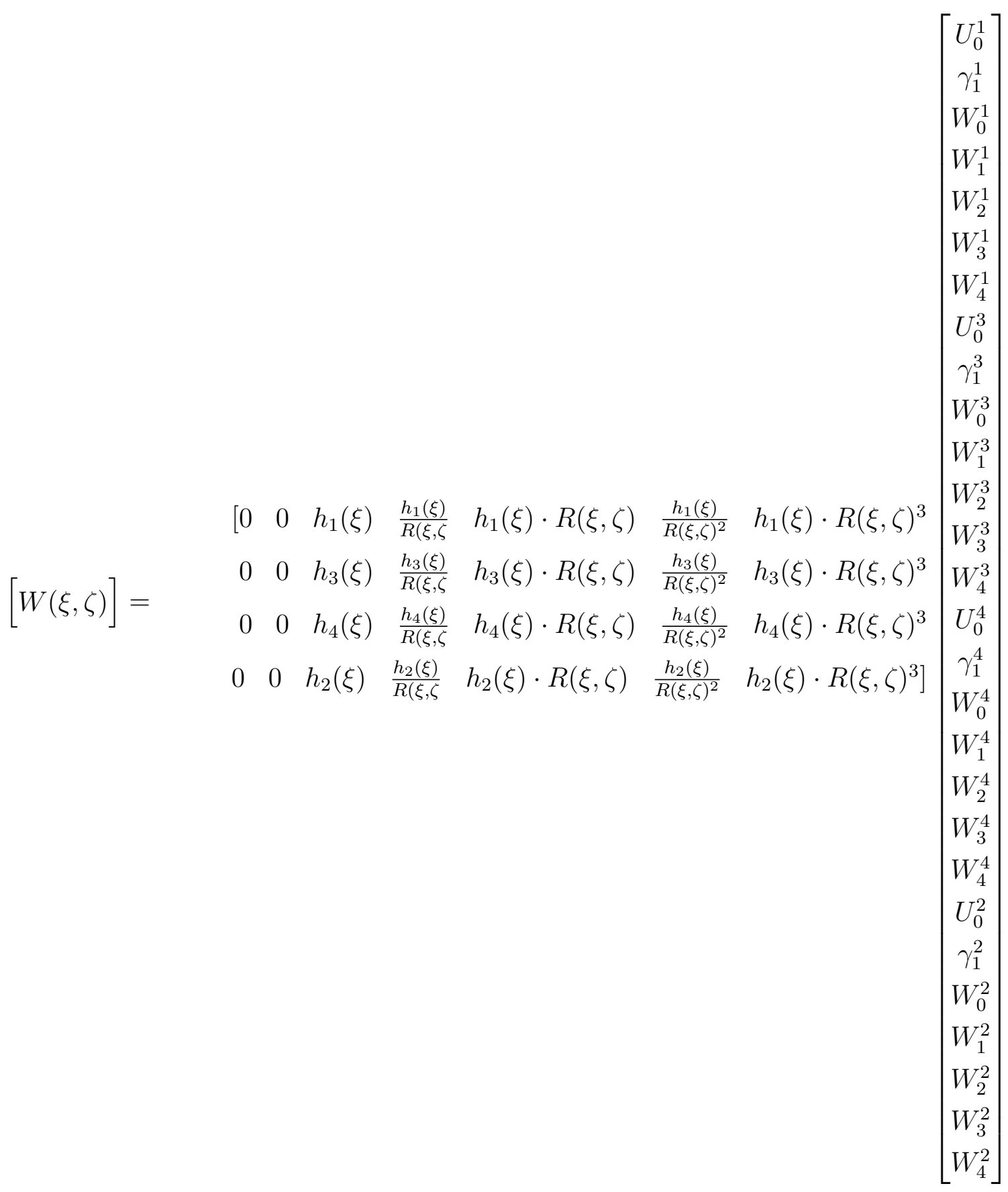




\section{7}

\section{Matriz B}

A partir do desenvolvimento descrito na seção acima encontrou-se a relação de deformação em função dos 7 graus liberdade para cada nó do elemento proposto. Assim, pode-se definir a matriz $\underline{B^{(n)}}$ elementar, resultado de colocar as deformações em função das variáveis do problema.

Portanto:

$$
\left[\begin{array}{c}
\epsilon_{\xi \xi} \\
\epsilon_{\theta \theta} \\
\epsilon_{\zeta \zeta} \\
\gamma_{\xi \zeta}
\end{array}\right]^{(n)}=\underline{B^{(n)}} \hat{U}^{(n)}
$$

em que a matriz $\underline{B}$ elementar apresenta 28 colunas, referente a quantidade de graus de liberdade totais no elemento, e 4 linhas, referente ao número de componentes de deformação do modelo. Para fins de visualização a matriz abaixo, com subíndice $i$, refere-se as submatrizes da matriz elementar $\underline{B^{(n)}}$, onde $i$ está é o nó de cada elemento

$$
B_{i}^{n}=\left[\begin{array}{lllllll}
a_{11}^{i} & a_{12}^{i} & a_{13}^{i} & a_{14}^{i} & a_{15}^{i} & a_{16}^{i} & a_{17}^{i} \\
a_{21}^{i} & a_{22}^{i} & a_{23}^{i} & a_{24}^{i} & a_{25}^{i} & a_{26}^{i} & a_{27}^{i} \\
a_{31}^{i} & a_{32}^{i} & a_{33}^{i} & a_{34}^{i} & a_{35}^{i} & a_{36}^{i} & a_{37}^{i} \\
a_{41}^{i} & a_{42}^{i} & a_{43}^{i} & a_{44}^{i} & a_{45}^{i} & a_{46}^{i} & a_{47}^{i}
\end{array}\right]
$$

Assim:

$$
B^{(n)}=\left[\begin{array}{llll}
B_{1}^{(n)} & B_{3}^{(n)} & B_{4}^{(n)} & B_{2}^{(n)}
\end{array}\right]
$$

Cada elemeto da submatriz elementar apresenta uma expressão obtida ao isolarmos cada grau de liberdade, seu deselvolvimento encontra-se no apêndice "Apêndice - Matriz B "na página 92. Logo:

$$
\begin{gathered}
a_{11}=\frac{1}{s^{\prime}}\left[\frac{\partial h_{i}}{\partial \xi}\right] \\
a_{12}=\frac{1}{s^{\prime}} \frac{\partial h_{i}}{\partial \xi}\left(\frac{h}{2} \zeta-\frac{h}{6} \zeta^{3}\right) \\
a_{13}=\frac{1}{s^{\prime}}\left[T^{3} \frac{\partial}{\partial \xi}\left(-\frac{2}{3 h^{2} s^{\prime}}\left[2 \frac{\partial h_{i}}{\partial \xi}\right]\right)\right] \\
a_{14}=-\frac{h \zeta^{2}}{4 s^{\prime 2}} \frac{\partial^{2} h_{i}}{\partial \xi^{2}}\left[\left(\frac{1}{R_{0}}-\frac{1}{R_{i}}\right) \frac{1}{2}+\left(\frac{1}{R_{0}}+\frac{1}{R_{i}}\right) \frac{\zeta}{3}\right] \\
a_{15}=-\frac{1}{s^{\prime 2}} \frac{\partial^{2} h_{i}}{\partial \xi^{2}} \frac{\zeta^{2}}{4}\left[\frac{h}{2}+\frac{2 \cdot R_{2}}{3} \zeta\right] \\
a_{16}=-\frac{1}{s^{\prime}} \frac{\partial^{2} h_{i}}{\partial \xi^{2}} \frac{\zeta^{2}}{4}\left[\left(\frac{1}{R_{0}^{2}}-\frac{1}{R_{i}^{2}}\right) \frac{1}{2}+\left(\frac{1}{R_{0}^{2}}+\frac{1}{R_{i}^{2}}\right) \frac{\zeta}{3}\right]
\end{gathered}
$$




$$
\begin{aligned}
& a_{17}=-\frac{1}{s^{\prime 2}} \frac{\partial^{2} h_{i}}{\partial \xi^{2}} \frac{\zeta^{2}}{4}\left[\left(R_{0}^{3}-R_{i}^{3}\right) \frac{1}{2}+\left(R_{0}^{3}+R_{i}^{3}\right) \frac{\zeta}{3}\right] \\
& a_{21}=0 \\
& a_{22}=0 \\
& a_{23}=\frac{h_{i}}{R_{2}+\frac{h}{2} \zeta} \\
& a_{24}=\frac{h_{i}}{\left(R_{2}+\frac{h}{2} \zeta\right)^{2}} \\
& a_{25}=h_{i} \\
& a_{26}=\frac{h_{i}}{\left(R_{2}+\frac{h}{2} \zeta\right)^{3}} \\
& a_{27}=h_{i} \cdot\left(R_{2}+\frac{h}{2} \zeta\right)^{2} \\
& a_{31}=0 \\
& a_{32}=0 \\
& a_{33}=0 \\
& a_{34}=-\frac{h_{i}}{\left(R_{2}+\frac{h}{2} \zeta\right)^{2}} \\
& a_{35}=h_{i} \\
& a_{36}=-\frac{2 h_{i}}{\left(R_{2}+\frac{h}{2} \zeta\right)^{3}} \\
& a_{37}=3 h_{i}\left(R_{2}+\frac{h}{2} \zeta\right)^{2} \\
& a_{41}=0 \\
& a_{42}=h_{i}\left(1-\zeta^{2}\right) \\
& a_{43}=\frac{1}{s^{\prime}} \frac{\partial h_{i}}{\partial \xi}\left(1-\zeta^{2}\right) \\
& a_{44}=\frac{1}{s^{\prime}} \frac{1}{R_{2}+\frac{h}{2} \zeta} \frac{\partial h_{i}}{\partial \xi}\left[\frac{1}{R}-\left(\frac{1}{R_{0}}-\frac{1}{R_{i}}\right) \frac{\zeta}{2}-\left(\frac{1}{R_{0}}+\frac{1}{R_{i}}\right) \frac{\zeta^{2}}{2}\right] \\
& a_{45}=\frac{1}{s^{\prime}} \frac{\partial h_{i}}{\partial \xi}\left(\left(R_{2}+\frac{h}{2} \zeta\right)-\left(R_{0}-R_{i}\right) \frac{\zeta}{2}-\left(R_{0}+R_{i}\right) \frac{\zeta^{2}}{2}\right)
\end{aligned}
$$




$$
\begin{aligned}
& a_{46}=\frac{1}{s^{\prime}} \frac{\partial h_{i}}{\partial \xi}\left(\frac{1}{\left(R_{2}+\frac{h}{2} \zeta\right)^{2}}-\left(\frac{1}{R_{0}^{2}}-\frac{1}{R_{i}^{2}}\right) \frac{\zeta}{2}-\left(\frac{1}{R_{0}^{2}}+\frac{1}{R_{i}^{2}}\right) \frac{\zeta^{2}}{2}\right) \\
& a_{47}=\frac{1}{s^{\prime}} \frac{\partial h_{i}}{\partial \xi} \frac{1}{2}\left(\left(R_{2}+\frac{h}{2} \zeta\right)^{3}-\left(R_{0}^{3}-R_{i}^{3}\right) \cdot \zeta-\left(R_{0}^{3}+R_{i}^{3}\right) \cdot \zeta^{2}\right)
\end{aligned}
$$




\section{8}

\section{Matriz C}

A matriz constitutiva $C$ estabelece a relação entre as componentes de tensão e de deformação. Para o problema aximétrico tem-se quatro componentes de tensões e de deformações. Assim, a matriz quadrada $C$ tem ordem quatro. Devido ao modelo do elemento corresponder ao caso isotrópico $C$ é simétrica, na forma

$$
\left[\begin{array}{c}
\sigma_{\xi \xi} \\
\sigma_{\theta \theta} \\
\sigma_{\zeta \zeta} \\
\tau_{\xi \zeta}
\end{array}\right]^{(n)}=\underline{C^{(n)}}\left[\begin{array}{c}
\epsilon_{\xi \xi} \\
\epsilon_{\theta \theta} \\
\epsilon_{\zeta \zeta} \\
\gamma_{\xi \zeta}
\end{array}\right]^{(n)}
$$

A matriz $C$ resulta da redução das equações contitutivas, dadas pela Lei de Hooke, expressando-se a condição

$$
\begin{gathered}
\epsilon_{\xi \xi}=\frac{\sigma_{\xi \xi}}{E}-\nu \frac{\sigma_{\theta \theta}}{E}-\nu \frac{\sigma_{\zeta \zeta}}{E} \\
\epsilon_{\theta \theta}=\frac{\sigma_{\theta \theta}}{E}-\nu \frac{\sigma_{\xi \xi}}{E}-\nu \frac{\sigma_{\zeta \zeta}}{E} \\
\epsilon_{\zeta \zeta}=\frac{\sigma_{\zeta \zeta}}{E}-\nu \frac{\sigma_{\xi \xi}}{E}-\nu \frac{\sigma_{\theta \theta}}{E} \\
\gamma_{\xi \zeta}=\frac{E}{2(1+\nu)} \sigma_{\xi \zeta}
\end{gathered}
$$

Rearranjando as equações acima obtem-se a matriz constitutiva elementar e o seu desenvolvimento completo encontra-se no apêndice à página 90 .

Portanto:

$$
\underline{C^{(n)}}=\frac{E(1-\nu)}{(1-2 \nu) \cdot(1+\nu)}\left[\begin{array}{cccc}
1 & \frac{\nu}{(1-\nu)} & \frac{\nu}{(1-\nu)} & 0 \\
\frac{\nu}{(1-\nu)} & 1 & \frac{\nu}{(1-\nu)} & 0 \\
\frac{\nu}{(1-\nu)} & \frac{\nu}{(1-\nu)} & 1 & 0 \\
0 & 0 & 0 & \frac{(1-2 \nu)}{2 \cdot(1-\nu)}
\end{array}\right]
$$




\section{9}

\section{Matriz de Rigidez K}

A matriz de rigidez relaciona o vetor de deslocamentos com o de carregamento aplicado, obtida através da integral

$$
\underline{K^{(n)}}=\int_{V^{(n)}} \underline{B^{(n) T}} \underline{C^{(n)}} \underline{B^{(n)}} d V^{(n)}
$$

Em elementos finitos, de forma a generalizar a equação 3-61 para geometrias quaisquer do elemento é utilizada a mudanças de coordenadas reduzindo-se os limites de integração ao intervalo de -1 a 1 . Com isso, nesta integral utiliza-se a transformação jacobiana.

Um outro aspecto importante é que por ser axissimétrico não é necessário integrar-se para todo ângulo da circunferência e, por conveniência numérica utiliza-se um radiano.

Portanto:

$$
\underline{K^{(n)}}=\int_{-1}^{1} \int_{-1}^{1} \underline{B(\xi, \zeta)^{(n) T}} \underline{C^{(n)}} \underline{B(\xi, \zeta)^{(n)}} \operatorname{det}(J) d V
$$

onde

$$
d V=R(\zeta) d \xi \quad d \zeta, \quad \operatorname{det}(J)=\frac{d z}{d \xi}
$$

e, assim,

$$
\underline{K^{(n)}}=\int_{-1}^{1} \int_{-1}^{1} \underline{B(\xi, \zeta)^{(n) T}} \underline{C^{(n)}} \underline{B(\xi, \zeta)^{(n)}} R(\zeta) \frac{d z}{d \xi} d \xi \quad d \zeta
$$




\subsection{0}

\section{Vetor Carregamento}

Em um problema de elementos finitos é necessário obter a equação (3-5) onde determina-se a matriz de rigidez e o vetor carregamento.

$\mathrm{O}$ vetor de carregamento permite a discretizaçao do forçamento que age sobre um corpo contabilizando a influência do carregamento em cada grau de liberdade do problema. Será modelado, a seguir, um forçamento de pressão (interna ou externa) e de rotação

\subsection{0 .1}

\section{Carregamento de pressão}

Para determinar-se o carregamento nodal resultante da pressão agindo na superfície interna e externa do cilindro deve-se integrar pela área a pressão multiplicada as funções interpoladoras. Assim:

sabendo que

$$
\hat{R}_{A}^{(n)}=\int_{A^{(n)}} \underline{H^{(n) T}} \underline{p^{(n)}} d A
$$

$$
d A=R_{A} \operatorname{det}(J) d \xi \quad, \quad \operatorname{det}(J)=\frac{d z}{d \xi}
$$

Tem-se

$$
\hat{R}_{A}^{(n)}=\int_{-1}^{1} \underline{H^{(n) T}}(\xi) \underline{p^{(n)}} R_{A} \frac{d z}{d \xi} d \xi
$$

Se houver pressão interna, $R_{A}$ é definido como:

$$
R_{A}=R_{2}-\frac{h}{2}
$$

e se for pressão externa, $R_{A}$ é definido como:

$$
R_{A}=R_{2}+\frac{h}{2}
$$

Assim:

$$
\hat{R}_{A}^{(n)}=\int_{-1}^{1} \underline{H^{(n) T}}(\xi) \underline{p_{\text {int }}^{(n)}}\left(R 2-\frac{h}{2}\right) \frac{d z}{d \xi} d \xi+\int_{-1}^{1} \underline{H^{(n) T}}(\xi) \underline{p_{\text {ext }}^{(n)}}\left(R 2+\frac{h}{2}\right) \frac{d z}{d \xi} d \xi
$$

onde: 


$$
\begin{aligned}
& \underline{H^{(n) T}}(\xi)=\left[\begin{array}{lllllll}
0 & 0 & h_{1} & \frac{h_{1}}{R_{A}} & h_{1} R_{A} & \frac{h_{1}}{R_{A}^{2}} & h_{1} R_{A}^{3}
\end{array}\right. \\
& \begin{array}{llllllll}
0 & 0 & h_{3} & \frac{h_{3}}{R_{A}} & h_{3} R_{A} & \frac{h_{3}}{R_{A}^{2}} & h_{3} R_{A}^{3}
\end{array} \\
& \begin{array}{llllllll}
0 & 0 & h_{4} & \frac{h_{4}}{R_{A}} & h_{4} R_{A} & \frac{h_{4}}{R_{A}^{2}} & h_{4} R_{A}^{3}
\end{array} \\
& \left.\begin{array}{lllllll}
0 & 0 & h_{2} & \frac{h_{2}}{R_{A}} & h_{2} R_{A} & \frac{h_{2}}{R_{A}^{2}} & h_{2} R_{A}^{3}
\end{array}\right]
\end{aligned}
$$

PNa integração numérica o vetor $V$ é tal que:

$$
R_{A}=\int_{-1}^{1} V d \xi
$$

Definindo-se o vetor $V$, onde os subíndices são as posições do mesmo:

$$
\begin{aligned}
& V_{1}=0 \\
& V_{2}=0 \\
& V_{3}=h_{i} p_{\text {int }}\left(R 2-\frac{h}{2}\right) \frac{d z}{d \xi}+h_{i} p_{\text {ext }}\left(R 2+\frac{h}{2}\right) \frac{d z}{d \xi} \\
& V_{3}=h_{i} \frac{d z}{d \xi}\left(p_{i n t} R_{i n t}+p_{\text {ext }} R_{\text {ext }}\right) \\
& V_{4}=\frac{h_{i}}{R_{\text {int }}} p_{\text {int }} R_{\text {int }} \frac{d z}{d \xi}+\frac{h_{i}}{R_{\text {ext }}} p_{\text {ext }} R_{\text {ext }} \frac{d z}{d \xi} \\
& V_{4}=h_{i} \frac{d z}{d \xi}\left(p_{\text {int }}+p_{\text {ext }}\right) \\
& V_{5}=h_{i} R_{\text {int }} p_{\text {int }} R_{\text {int }} \frac{d z}{d \xi}+h_{i} R_{\text {ext }} p_{\text {ext }} R_{\text {ext }} \frac{d z}{d \xi} \\
& V_{5}=h_{i} \frac{d z}{d \xi}\left(p_{\text {int }} R_{\text {int }}^{2}+p_{\text {ext }} R_{\text {ext }}^{2}\right) \\
& V_{6}=\frac{h_{i}}{R_{\text {int }}^{2}} p_{\text {int }} R_{\text {int }} \frac{d z}{d \xi}+\frac{h_{i}}{R_{\text {ext }}^{2}} p_{\text {ext }} R_{\text {ext }} \frac{d z}{d \xi} \\
& V_{6}=h_{i} \frac{d z}{d \xi}\left(\frac{P_{i n t}}{R_{\text {int }}}+\frac{p_{\text {ext }}}{R_{\text {ext }}}\right) \\
& V_{7}=h_{i} R_{\text {int }}^{3} p_{\text {int }} R_{\text {int }} \frac{d z}{d \xi}+h_{i} R_{\text {ext }}^{3} p_{\text {ext }} R_{\text {ext }} \frac{d z}{d \xi} \\
& V_{7}=h_{i} \frac{d z}{d \xi}\left(p_{\text {int }} R_{\text {int }}^{4}+p_{\text {ext }} R_{\text {ext }}^{4}\right)
\end{aligned}
$$




\subsection{0 .2}

\section{Carregamento de rotação}

Quando submetido à rotação, o vetor força de corpo é formado como carregamento externo. Desta forma,

onde:

$$
\hat{R}_{B}^{(n)}=\int_{V^{(n)}} \underline{H^{(n) T}} \underline{f}^{B} d V
$$

$$
\left.\begin{array}{c}
\underline{f}^{B}=\rho \omega^{2} R(\xi, \zeta) \\
\hat{R}_{B}^{(n)}=\int_{V^{(n)}} \underline{H^{(n) T}}\left[\begin{array}{c}
0 \\
0 \\
0 \\
\rho \omega^{2}
\end{array}\right] d V(\xi, \zeta)
\end{array}\right] d V
$$

Usando a equação 3-29.

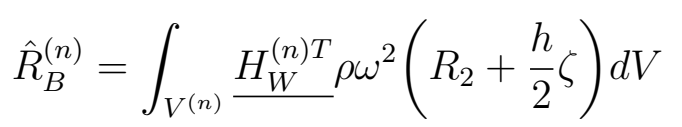

$\mathrm{Ou}$

$$
\hat{R}_{B}^{(n)}=\int_{-1}^{1} \int_{-1}^{1} \frac{H_{W}^{(n) T}}{\rho} \rho \omega^{2}\left(R_{2}+\frac{h}{2} \zeta\right)^{2} \frac{d z}{d \xi} d \xi d \zeta
$$

da integração numérica define-se o vetor $V$ :

$$
\begin{gathered}
\hat{R}_{B}^{(n)}=\int_{-1}^{1} \int_{-1}^{1} V d \xi d \zeta \\
V_{1}=0 \\
V_{2}=0 \\
V_{3}=h_{i} \rho \omega^{2}\left(R_{2}+\frac{h}{2} \zeta\right)^{2} \frac{d z}{d \xi} \\
\left(R_{2}+\frac{h}{2} \zeta\right)^{2} \rho \omega^{2}\left(R_{2}+\frac{h}{2} \zeta\right)^{2} \frac{d z}{d \xi} \\
V_{4}=h_{i} \rho \omega^{2}\left(R_{2}+\frac{h}{2} \zeta\right)^{\frac{d z}{d \xi}} \\
V_{5}=h_{i}\left(R_{2}+\frac{h}{2} \zeta\right) \rho \omega^{2}\left(R_{2}+\frac{h}{2} \zeta\right)^{2} \frac{d z}{d \xi} \\
V_{5}=h_{i} \rho \omega^{2}\left(R_{2}+\frac{h}{2} \zeta\right)^{3} \frac{d z}{d \xi}
\end{gathered}
$$




$$
\begin{gathered}
V_{6}=\frac{h_{i}}{\left(R_{2}+\frac{h}{2} \zeta\right)^{2}} \rho \omega^{2}\left(R_{2}+\frac{h}{2} \zeta\right)^{2} \frac{d z}{d \xi} \\
V_{6}=h_{i} \rho \omega^{2} \frac{d z}{d \xi} \\
V_{7}=h_{i}\left(R_{2}+\frac{h}{2} \zeta\right)^{3} \rho \omega^{2}\left(R_{2}+\frac{h}{2} \zeta\right)^{2} \frac{d z}{d \xi} \\
V_{7}=h_{i} \rho \omega^{2}\left(R_{2}+\frac{h}{2} \zeta\right)^{5} \frac{d z}{d \xi}
\end{gathered}
$$




\subsection{1}

\section{Condição de Continuidade}

Ao se utilizar uma metodologia de elementos finitos enriquecisdos, desejase encontrar resultados bastante próximos do esperado com poucos elementos. Assim, é importante garantir primeiras derivadas iguais na fronteira dos elementos.

$$
\zeta=-1 \underbrace{\mid}_{\substack{2 \\ \xi=+1}} \zeta=+1
$$

Logo, deve-se garantir duas restrições:

- Ponto 1

$$
\left.\left(\frac{1}{s^{\prime}} \frac{\partial W}{\partial \xi}\right)_{\xi=+1}^{(n)}\right|_{\zeta=+1}=\left.\left(\frac{1}{s^{\prime}} \frac{\partial W}{\partial \xi}\right)_{\xi=-1}^{(n+1)}\right|_{\zeta=+1}
$$

A restrição precisa ser escrita da forma $g_{i}(\hat{X})=0$, logo

$$
g_{1}=\left[\left(\frac{1}{s^{\prime}} \frac{\partial W}{\partial \xi}\right)_{\xi=+1}^{(n)}-\left(\frac{1}{s^{\prime}} \frac{\partial W}{\partial \xi}\right)_{\xi=-1}^{(n+1)}\right]_{\zeta=+1}
$$

- Ponto 2

$$
\left.\left(\frac{1}{s^{\prime}} \frac{\partial W}{\partial \xi}\right)_{\xi=+1}^{(n)}\right|_{\zeta=-1}=\left.\left(\frac{1}{s^{\prime}} \frac{\partial W}{\partial \xi}\right)_{\xi=-1}^{(n+1)}\right|_{\zeta=-1}
$$

A restrição precisa ser escrita da forma $g_{i}(\hat{X})=0$, logo

$$
g_{1}=\left[\left(\frac{1}{s^{\prime}} \frac{\partial W}{\partial \xi}\right)_{\xi=+1}^{(n)}-\left(\frac{1}{s^{\prime}} \frac{\partial W}{\partial \xi}\right)_{\xi=-1}^{(n+1)}\right]_{\zeta=-1}
$$

O método da penalidade, comumente utilizado em problemas de otimização, permite a minimização de uma função $F(\hat{X})$ a partir de uma pseudofunção $P(\hat{X}, \alpha)$, dadas suas restrições $g_{i}(\hat{X})=0$. O parâmetro $\alpha$ deve ser 
ajustado a fim de equilibrar a ordem de grandeza de ambas parcelas.

Portanto:

$$
P(\hat{X}, \alpha)=F(\hat{X})+\frac{\alpha}{2} \sum_{i=1}^{N}\left[g_{i}(\hat{X})\right]^{2}
$$

Deseja-se minimizar o funcional $\Pi$ de elementos finitos, dadas as restrições $g_{i}$, criando assim um novo funcional, dado por $\Pi^{\prime}$ :

$$
\begin{gathered}
P(\hat{X}, \alpha)=\Pi^{\prime} \\
F(\hat{X})=\Pi
\end{gathered}
$$

Portanto:

$$
\Pi^{\prime}=\Pi+\frac{1}{2} \alpha \cdot g_{1}^{2}+\frac{1}{2} \alpha \cdot g_{2}^{2}
$$

Fazendo o variacional, tem-se:

$$
\begin{gathered}
\delta \Pi^{\prime}=\delta \underline{u}^{T} \underline{K} \underline{u}+\delta \underline{u}^{T} B_{1}^{T} \alpha B_{1} \underline{u}+\delta \underline{u}^{T} B_{2}^{T} \alpha B_{2} \underline{u} \\
\delta \Pi^{\prime}=\delta \underline{u}^{T}\left(K+K_{1}^{F}+K_{2}^{F}\right) \underline{u}
\end{gathered}
$$

Lembrando que:

$\frac{\partial W}{\partial \xi}=\frac{\partial}{\partial \xi}\left(\sum h_{i} W_{0}^{i}+\frac{1}{R} \sum h_{i} W_{1}^{i}+R \sum h_{i} W_{2}^{i}+\frac{1}{R^{2}} \sum h_{i} W_{3}^{i}+R^{3} \sum h_{i} W_{4}^{i}\right)$

Nesse caso, a penalidade não poderá ser escrita por apenas um elemento, posto que a mesma é imposta entre dois elementos, $(n)$ e $(n+1)$. Dessa forma, a penalidade será uma matriz para cada união entre dois elementos, o que forçará que tenham primeiras derivadas iguais na junção. Nesse sentido, o vetor $B$ deve conter 49 posições, oriundas dos 7 nós num problema de dois elementos, cada qual com 7 graus de liberdade.

Para definir o vetor $B(\xi, \zeta)$, será usada uma variável adicional $(b)$ que expressará o valor pelo qual as derivadas serão aplicadas. Segue a tabela abaixo:

\begin{tabular}{|c|c|c|}
\hline & $B(\xi, \zeta)$ & $b=\zeta$ \\
\hline$B_{1}$ & $B(\xi, 1)$ & $b=1$ \\
\hline$B_{2}$ & $B(\xi,-1)$ & $b=-1$ \\
\hline
\end{tabular}

O vetor $B_{n}$, para facilitar sua visualização, será composto de sub-vetores, que estão associados a cada nó. Assim, o vetor $d^{i}$ tem 7 posições e apresenta subíndece $i$ que corresponde a função lagrangeana que deve ser utilizada. Além disso, está em função de $a$ e $b$, vide equação abaixo. O sub-vetor $c$ será utilizado no nó em conjunto dos elementos. Assim: 


$$
\begin{aligned}
& B_{n}^{T}(b)= \\
& {\left[d^{1}(a=+1, b) \quad d^{3}(a=+1, b) \quad d^{4}(a=+1, b) \quad c(b)\right.} \\
& \left.d^{3}(a=-1, b) \quad d^{4}(a=-1, b) \quad d^{2}(a=-1, b)\right]
\end{aligned}
$$

O sub-vetor $d^{i}(a, b)$ :

- Isolar $U_{0}^{i}$ em $(n)$ :

$$
a_{1}^{i}=0
$$

- Isolar $\gamma_{1}^{i}$ em $(n)$ :

$$
a_{2}^{i}=0
$$

- Isolar $W_{0}^{i}$ em $(n)$ :

$$
a_{3}^{i}=\left[\left.\frac{1}{s^{\prime}} \frac{\partial h_{i}}{\partial \xi}\right|_{\xi=a}\right]_{\zeta=b}
$$

- Isolar $W_{1}^{i}$ em $(n)$ :

$$
a_{4}^{i}=\left[\left(\frac{1}{s^{\prime}} \frac{1}{R} \frac{\partial h_{i}}{\partial \xi}\right)_{\xi=a}\right]_{\zeta=b}
$$

- Isolar $W_{2}^{i}$ em $(n)$ :

$$
a_{5}^{i}=\left[\left(\frac{R}{s^{\prime}} \frac{1}{R} \frac{\partial h_{i}}{\partial \xi}\right)_{\xi=a}\right]_{\zeta=b}
$$

- Isolar $W_{3}^{i}$ em $(n)$ :

$$
a_{6}^{i}=\left[\left(\frac{1}{s^{\prime} R^{2}} \frac{1}{R} \frac{\partial h_{i}}{\partial \xi}\right)_{\xi=a}\right]_{\zeta=b}
$$

- Isolar $W_{4}^{i}$ em $(n)$ :

$$
a_{7}^{i}=\left[\left(\frac{R^{3}}{s^{\prime}} \frac{1}{R} \frac{\partial h_{i}}{\partial \xi}\right)_{\xi=a}\right]_{\zeta=b}
$$

O sub-vetor $c(b)$ :

- Isolar $U_{0}^{i}$ em $(n)$ e $(n+1)$ :

$$
c_{1}=0
$$

- Isolar $\gamma_{1}^{i}$ em $(n)$ e $(n+1)$ :

$$
c_{2}=0
$$

- Isolar $W_{0}^{i}$ em $(n)$ e $(n+1)$ :

$$
c_{3}=\left[\left(\frac{1}{s^{\prime}} \frac{\partial h_{1}}{\partial \xi}\right)_{\xi=+1}-\left(\frac{1}{s^{\prime}} \frac{\partial h_{2}}{\partial \xi}\right)_{\xi=-1}\right]_{\zeta=b}
$$

- Isolar $W_{1}^{i}$ em $(n)$ e $(n+1)$ :

$$
c_{4}=\left[\left(\frac{1}{R s^{\prime}} \frac{\partial h_{1}}{\partial \xi}\right)_{\xi=+1}-\left(\frac{1}{R s^{\prime}} \frac{\partial h_{2}}{\partial \xi}\right)_{\xi=-1}\right]_{\zeta=b}
$$

- Isolar $W_{2}^{i}$ em $(n)$ e $(n+1)$ :

$$
c_{5}=\left[\left(\frac{R}{s^{\prime}} \frac{\partial h_{1}}{\partial \xi}\right)_{\xi=+1}-\left(\frac{R}{s^{\prime}} \frac{\partial h_{2}}{\partial \xi}\right)_{\xi=-1}\right]_{\zeta=b}
$$


- Isolar $W_{3}^{i}$ em $(n)$ e $(n+1)$ :

$$
c_{6}=\left[\left(\frac{1}{R^{2} s^{\prime}} \frac{\partial h_{1}}{\partial \xi}\right)_{\xi=+1}-\left(\frac{1}{R^{2} s^{\prime}} \frac{\partial h_{2}}{\partial \xi}\right)_{\xi=-1}\right]_{\zeta=b}
$$

- Isolar $W_{4}^{i}$ em $(n)$ e $(n+1)$ :

$$
c_{7}=\left[\left(\frac{R^{3}}{s^{\prime}} \frac{\partial h_{1}}{\partial \xi}\right)_{\xi=+1}-\left(\frac{R^{3}}{s^{\prime}} \frac{\partial h_{2}}{\partial \xi}\right)_{\xi=-1}\right]_{\zeta=b}
$$

Seguindo o procedimento acima, os vetores $B$ podem ser encontrados. Vale comentar como é feita a determinação do parâmetro $\alpha$. Foi assumido o mesmo valor de $\alpha$ para as quatro penalidades. Assim, fazendo-se o produto de $B^{T} \cdot B$, encontra-se $K^{\prime}$ (sem a multiplicação por $\alpha$ ). O parâmetro $\alpha$ pode ser ajustado dividindo o maior valor pertencente a matriz de rigidez do problema pelo maior valor da matriz fruto do somatório dos $K^{\prime}$. Logo, a penalidade será imposta. 
3.12

\section{Condição de Fixação}

A fixação é imposta quando há condição de engaste na extremidade. Assim, será utilizada uma penalidade que forçará derivada nula do deslocamento radial na seção engastada.

- Engaste inferior

$$
\begin{gathered}
\left.\left.{ }_{\mid}||_{\zeta=-1}\right|_{\zeta=+1} \zeta\right|_{\zeta=+1}=0 \\
\left.\left[\left(\frac{\partial W}{\partial \xi}\right)_{\xi=+1}^{(i)}\right]_{\zeta=+1}^{(i)}\right]_{\xi=+1}=0 \\
{\left[\left(\frac{\partial W}{\partial \xi}\right]_{\zeta=-1}\right.}
\end{gathered}
$$

A restrição precisa ser escrita na forma $g_{i}(\hat{X})=0$ para aplicar a penalidade. Portanto:

$$
\begin{aligned}
& g_{11}=\left[\left(\frac{\partial W}{\partial \xi}\right)_{\xi=+1}^{(i)}\right]_{\zeta=+1} \\
& g_{12}=\left[\left(\frac{\partial W}{\partial \xi}\right)_{\xi=+1}^{(i)}\right]_{\zeta=-1}
\end{aligned}
$$

- Engaste superior

$$
\begin{aligned}
& \zeta=-1</ / 1 / \zeta=+1 \\
& \xi=+1 \\
& {\left[\left(\frac{\partial W}{\partial \xi}\right)_{\xi=-1}^{(i)}\right]_{\zeta=+1}=0}
\end{aligned}
$$




$$
\left[\left(\frac{\partial W}{\partial \xi}\right)_{\xi=-1}^{(i)}\right]_{\zeta=-1}=0
$$

A restrição precisa ser escrita na forma $g_{i}(\hat{X})=0$ para aplicar a penalidade. Portanto:

$$
\begin{aligned}
& g_{21}=\left[\left(\frac{\partial W}{\partial \xi}\right)_{\xi=-1}^{(i)}\right]_{\zeta=+1} \\
& g_{22}=\left[\left(\frac{\partial W}{\partial \xi}\right)_{\xi=-1}^{(i)}\right]_{\zeta=-1}
\end{aligned}
$$

O método da penalidade, comumente utilizado em problemas de otimização, permite a minimização de uma função $F(\hat{X})$ a partir de uma pseudofunção $P(\hat{X}, \alpha)$, dadas suas restrições $g_{i}(\hat{X})=0$. O parâmetro $\alpha$ deve ser ajustado a fim de equilibrar a ordem de grandeza de ambas parcelas.

Portanto:

$$
P(\hat{X}, \alpha)=F(\hat{X})+\frac{\alpha}{2} \sum_{i=1}^{N}\left[g_{i}(\hat{X})\right]^{2}
$$

Deseja-se minimizar o funcional $\Pi$ de elementos finitos dadas as restrições $g_{i}$, criando, assim, um novo funcional, dado por $\Pi^{\prime}$ :

$$
\begin{gathered}
P(\hat{X}, \alpha)=\Pi^{\prime} \\
F(\hat{X})=\Pi
\end{gathered}
$$

Portanto:

$$
\Pi^{\prime}=\Pi+\frac{1}{2} \alpha\left(g_{11}\right)^{2}+\frac{1}{2} \alpha\left(g_{12}\right)^{2}+\frac{1}{2} \alpha\left(g_{21}\right)^{2}+\frac{1}{2} \alpha\left(g_{22}\right)^{2}
$$

Fazendo o variacional, tem-se:

$$
\begin{gathered}
\delta \Pi^{\prime}=\delta \underline{u}^{T} \underline{K} \underline{u}+\delta \underline{u}^{T} B_{11}^{T} \alpha B_{11} \underline{u}+\delta \underline{u}^{T} B_{12}^{T} \alpha B_{12} \underline{u}+ \\
\delta \underline{u}^{T} B_{21}^{T} \alpha B_{21} \underline{u}+\delta \underline{u}^{T} B_{22}^{T} \alpha B_{22} \underline{u} \\
\delta \Pi^{\prime}=\delta \underline{u}^{T}\left(K+K_{11}^{F}+K_{12}^{F}+K_{21}^{F}+K_{22}^{F}\right) \underline{u}
\end{gathered}
$$

Lembrando que:

$$
\frac{\partial W}{\partial \xi}=\frac{\partial}{\partial \xi}\left(\sum h_{i} W_{0}^{i}+\frac{1}{R} \sum h_{i} W_{1}^{i}+R \sum h_{i} W_{2}^{i}+\frac{1}{R^{2}} \sum h_{i} W_{3}^{i}+R^{3} \sum h_{i} W_{4}^{i}\right)
$$

Para definir o vetor $B(\xi, \zeta)$, serão usadas duas variáveis adicionais $(a$ e $b)$ que expressarão os valores pelos quais as derivadas serão aplicadas. Segue a 
tabela abaixo:

\begin{tabular}{|c|c|c|c|}
\hline & $B(\xi, \zeta)$ & $a=\xi$ & $b=\zeta$ \\
\hline$B_{11}$ & $B(1,1)$ & $a=1$ & $b=1$ \\
\hline$B_{12}$ & $B(1,-1)$ & $a=1$ & $b=-1$ \\
\hline$B_{21}$ & $B(-1,1)$ & $a=-1$ & $b=1$ \\
\hline$B_{22}$ & $B(-1,-1)$ & $a=-1$ & $b=-1$ \\
\hline
\end{tabular}

- Isolar $U_{0}^{i}$ :

$$
B_{1}=0
$$

- Isolar $\gamma_{1}^{i}$ :

$$
B_{2}=0
$$

- Isolar $W_{0}^{i}$ :

$$
B_{3}=\left.\frac{\partial h_{i}}{\partial \xi}\right|_{\xi=a}
$$

- Isolar $W_{1}^{i}$ :

$$
B_{4}=\left.\frac{1}{R} \frac{\partial h_{i}}{\partial \xi}\right|_{\xi=a}=\left.\frac{1}{R_{m}+\left.\frac{h}{2} \zeta\right|_{\zeta=b}} \frac{\partial h_{i}}{\partial \xi}\right|_{\xi=a}
$$

- Isolar $W_{2}^{i}$ :

$$
B_{5}=\left.R \frac{\partial h_{i}}{\partial \xi}\right|_{\xi=a}=\left.\left[R_{m}+\left.\frac{h}{2} \zeta\right|_{\zeta=b}\right] \frac{\partial h_{i}}{\partial \xi}\right|_{\xi=a}
$$

- Isolar $W_{3}^{i}$ :

$$
B_{6}=\left.\frac{1}{R^{2}} \frac{\partial h_{i}}{\partial \xi}\right|_{\xi=a}=\left.\frac{1}{\left[R_{m}+\left.\frac{h}{2} \zeta\right|_{\zeta=b}\right]^{2}} \frac{\partial h_{i}}{\partial \xi}\right|_{\xi=a}
$$

- Isolar $W_{4}^{i}$ :

$$
B_{7}=\left.R^{3} \frac{\partial h_{i}}{\partial \xi}\right|_{\xi=a}=\left.\left[R_{m}+\left.\frac{h}{2} \zeta\right|_{\zeta=b}\right]^{3} \frac{\partial h_{i}}{\partial \xi}\right|_{\xi=a}
$$

Seguindo o procedimento acima, os vetores $B$ podem ser encontrados. Vale comentar como é feita a determinação do parâmetro $\alpha$. Foi assumido o mesmo valor de $\alpha$ para as quatro penalidades. Assim, fazendo o produto de $B^{T} \cdot B$, encontra-se $K^{\prime}$ (sem a multiplicação por $\alpha$ ). O parâmetro $\alpha$ pode ser ajustado dividindo o maior valor pertencente a matriz de rigidez do problema pelo maior valor da matriz fruto do somatório dos $K^{\prime}$. Logo, a penalidade será imposta. 


\section{Programa}

A partir do modelo proposto, um programa em "C" foi elaborado com a finalidade de automatizar os cálculos necessários para se realizar a análise de tensões em um vaso de pressão. Assim, fornecendo-se a quantidade de elementos desejados e os carregamentos atuantes no corpo, obtêm-se com grande eficiência os resultados desejados.

Para compreensão do próximo capítulo, vale explicar como se aplicavam as condições de contorno no problema. Para isso, uma tabela era feita onde, na primeira coluna, a numeração dos nós eram dispostas. As seguintes colunas usavam 1 para travar uma condição de contorno e 0 para deixá-la livre. A segunda coluna refere-se ao travamento do deslocamento radial, a terceira coluna ao travamento axial e a quarta ao travamento da rotação na seção reta. Para análise futura dos resultados, será usada uma tabela que representará as condições de contorno. Esquematicamente:

\begin{tabular}{|c|c|c|c|}
\hline & \multicolumn{3}{|c|}{ Condição de contorno } \\
\hline Nós & $\begin{array}{c}\text { Deslocamento } \\
\text { radial }\end{array}$ & $\begin{array}{c}\text { Deslocamento } \\
\text { axial }\end{array}$ & $\begin{array}{c}\text { Rotação da } \\
\text { seção reta }\end{array}$ \\
\hline 1 & 1 ou 0 & 1 ou 0 & 1 ou 0 \\
\hline$\vdots$ & $\vdots$ & $\vdots$ & $\vdots$ \\
\hline
\end{tabular}




\section{Resultados Numéricos}

Neste capítulo, serão avaliados os resultados numéricos obtidos a partir do programa desenvolvido. Em seguida, serão comparados com soluções conhecidas da literatura ou provindos do programa comercial de elementos finitos Ansys.

\section{1}

\section{Cilindro sujeito à pressão}

Nesta seção, será estudado o comportamento de cilindros sujeito à pressão interna ou externa para duas diferentes condições de contorno, que serão expressas nas duas subseções abaixo. A primeira considera o travamento do deslocamento axial e da rotação da seção da base do cilindro, enquanto a segunda considera cilindro livre.

Para as dois casos de condição de contorno, os dados numéricos utilizados são:

- Módulo de elasticidade: $200 \mathrm{GPa}$

- Coeficiente de Poison: $0.3 \quad$ Logo:

- Espessura: $50 \mathrm{~m}$ - Raio interno: $25 \mathrm{~m}$

- Raio médio: $50 \mathrm{~m}$ - Raio externo: $75 \mathrm{~m}$

- Pressão interna: $12 \mathrm{MPa}$

\section{1 .1}

Cilindro, com travamento de deslocamento e restrição de rotação na seção reta da base

Conforme já mencionado no capítulo "Programa", para impor a condição de contorno descrita no título desta subseção, deve-se utilizar o quadro esquemático abaixo:

\begin{tabular}{|c|c|c|c|}
\hline Nós & \multicolumn{3}{|c|}{ Condições de contorno } \\
\hline 1 & 0 & 1 & 1 \\
\hline 2 & 0 & 0 & 0 \\
\hline 3 & 0 & 0 & 0 \\
\hline 4 & 0 & 0 & 0 \\
\hline
\end{tabular}


A seguir, dois diferentes casos serão estudados. O primeiro considerará o carregamento de pressão interna, enquanto o segundo de pressão externa.

Cilindro, com travamento de deslocamento e restrição de rotação na seção reta da base, sujeito a pressão interna de $12 \mathrm{MPa}$ - Solução de Lamé

O primeiro caso que será estudado é a solução de Lamé, importante resultado para validar o programa elaborado. Observando a solução analítica desenvolvida por Lamé, percebe-se o travamento da deformação axial ao longo de toda espessura, fato que pode ser verificado na equação 1-7. Os gráficos abaixo foram obtidos utilizando como condição de contorno o travamento axial no nó central com a restrição de rotação da seção reta na extremidade do elemento. Garante-se, assim, a inexistência de deslocamento axial ao longo de toda espessura radial.

Tratando-se de uma abordagem utilizando elementos finitos enriquecidos, poucos elementos garantem convergência do resultado. Assim, nesta análise será usado apenas um elemento.

Os gráficos 5.1 e 5.2 apresentam os resultados da tensão radial e circunferencial, respectivamente. Nota-se que a solução analítica é resgatada quando aplicam-se as mesmas condições de contorno utilizadas em seu desenvolvimento. As curvas analíticas são obtidas pelas equações 1-26 e 1-27.

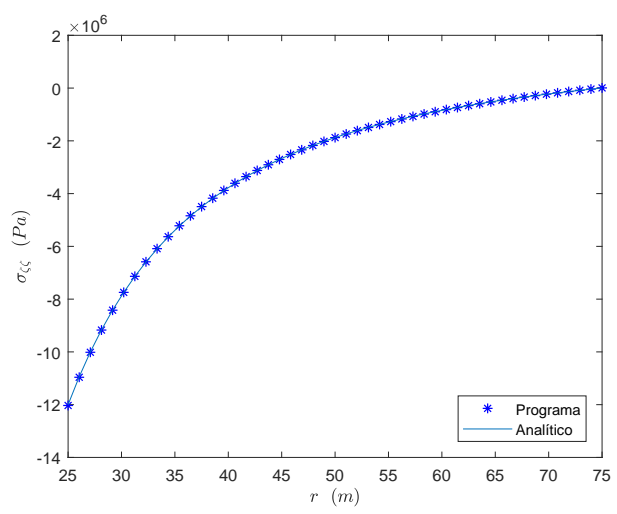

Figura 5.1: Tensão radial nó 1

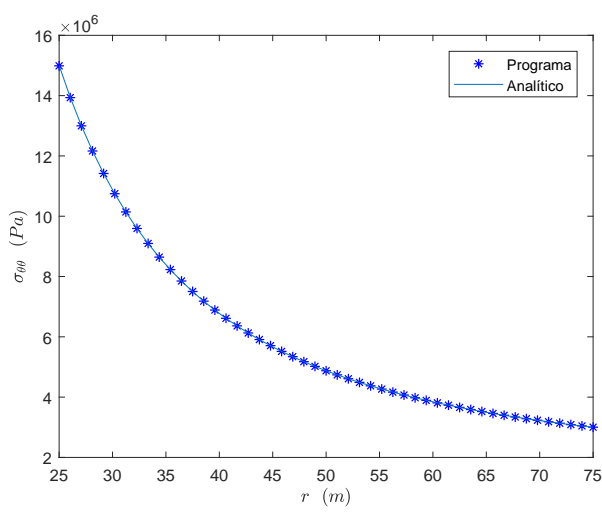

Figura 5.2: Tensão circunferencial nó 1

Uma análise importante que se deve observar no gráfico 5.1 acima é o valor da tensão radial no raio interno e no raio externo do vaso de pressão. Para a parede interna, percebe-se o mesmo valor numérico com sinal contrário da pressão interna aplicada no carregamento, enquanto na parede externa tensão nula. Este resultado está conforme o esperado, já que as superfícies, interna e externa do vaso de pressão, devem estar em equilíbrio estático. 
Os resultados das deformações radiais e circunferenciais são apresentados nas figuras 5.3 e 5.4 abaixos. Novamente, é possível comparar com as soluções analíticas desenvolvidas no primeiro capítulo, percebendo-se, portanto, grande proximidade entre os modelos analítico e numérico.

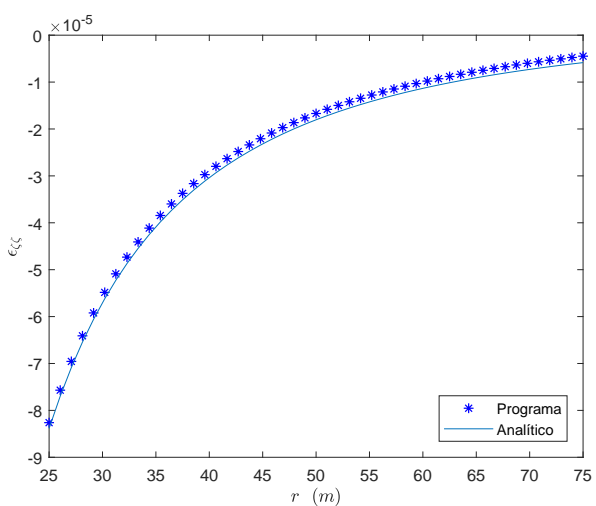

Figura 5.3: Deformação radial nó 1 Figura 5.4: Deformação circunferencial nó 1

A figura 5.5 abaixo é um importante parâmetro de verificação da solução numérica pelo programa elaborado. Istso porque os deslocamentos são a incógnita de um problema de elementos finitos. Nesse sentido, são os primeiros resultados a se analisar. Nota-se, portanto, que os resultados estão de acordo com a formulação analítica.

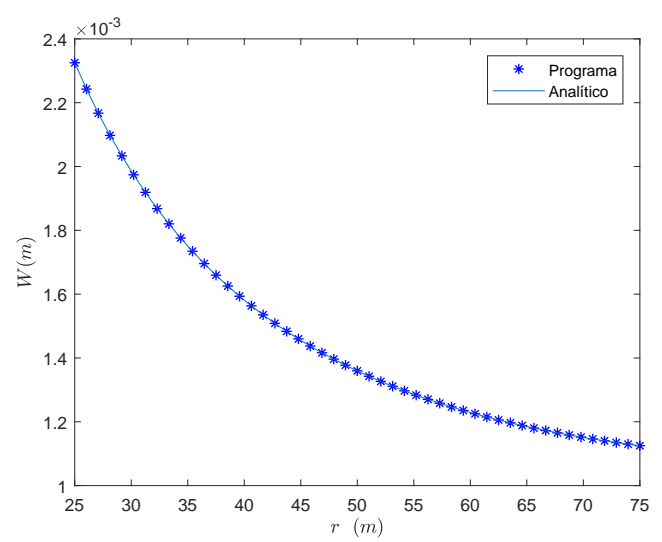

Figura 5.5: Deslocamento radial nó 1 
Cilindro, com travamento de deslocamento e restrição de rotação na seção reta da base, sujeito a pressão externa de $12 \mathrm{MPa}$ - Solução de Lamé

De maneira similar ao primeiro caso, a solução analítica para pressão externa é obtida pelas equações (1-23) e (1-25). Ao se comparar com o resultado analítico, nota-se a superposição das curvas.

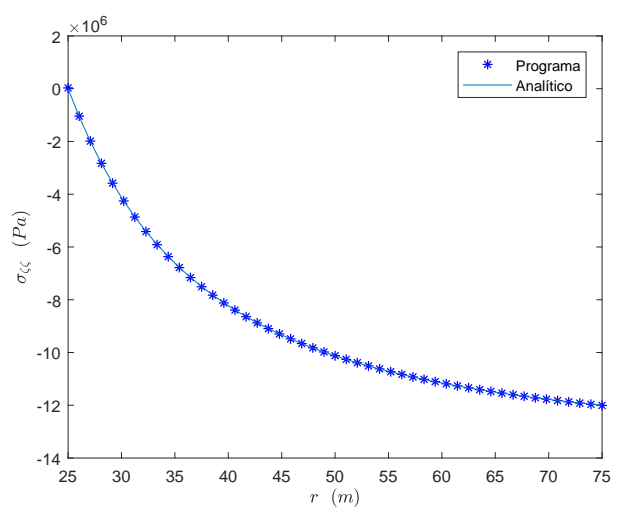

Figura 5.6: Tensão radial nó 1

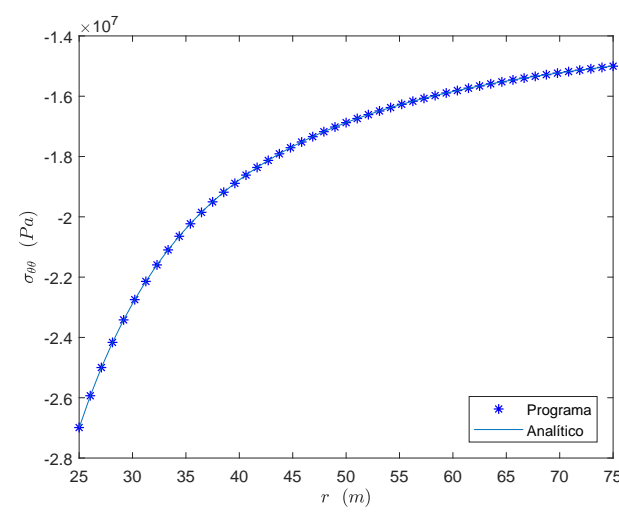

Figura 5.7: Tensão circunferencial nó 1

Novamente, percebe-se o equilíbrio das superfícies interna e externa do vaso. Para a parede interna, tem-se pressão nula, enquanto para a externa sinal contrário ao valor da pressão.

O gráfico 5.8 abaixo compara o deslocamento radial obtido do modelagem numérica com o seu valor do desenvolvimento analítico. Como esperado, os resultados são bastante próximos.

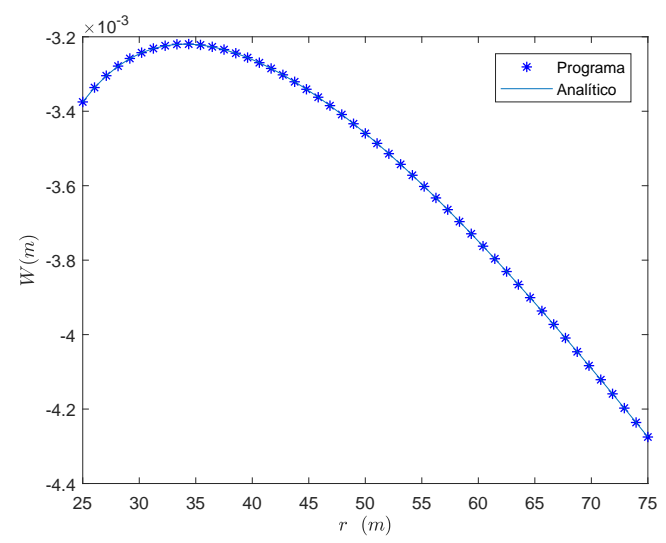

Figura 5.8: Deslocamento radial nó 1 


\section{1 .2}

\section{Cilindro livre}

Esta subseção refere-se a cilindro livre, segunda condição de contorno estudada para cilindros sujeitos à pressão. Para isso, implementa-se no programa conforme a tabela abaixo.

\begin{tabular}{|c|c|c|c|}
\hline Nós & \multicolumn{3}{|c|}{ Condições de contorno } \\
\hline 1 & 0 & 0 & 0 \\
\hline 2 & 0 & 0 & 0 \\
\hline 3 & 0 & 0 & 0 \\
\hline 4 & 0 & 0 & 0 \\
\hline
\end{tabular}

Novamente, para a condição de contorno imposta, primeiro será estudado o efeito da pressão interna e, em seguida, o da pressão externa.

\section{Cilindro livre sujeito a pressão interna de $12 \mathrm{MPa}$}

O próximo caso a ser analisado trata-se da aplicação de uma pressão interna de $12 \mathrm{MPa}$ num cilindro espesso sob condição de contorno livre, ou seja, sem nenhuma restrição ao deslocamento. Para efeito de comparação, o problema foi também simulado no Ansys. Ao se observar os gráficos 5.9. 5.10, 5.11 e 5.12 abaixo, nota-se a superposição de ambos os resultados, demonstrando exatidão na metodologia numérica utilizada. Além disso, é importante verificar se as condições de contorno naturais são respeitadas nos resultados obtidos. Logo, tomando o gráfico de tensões radiais, figura número 5.9. observa-se que a tensão radial na parede interna é $12 M P a$ compressiva, enquanto na externa é zero - fato esperado, pois o equilíbrio em ambas superfícies deve ser respeitado.

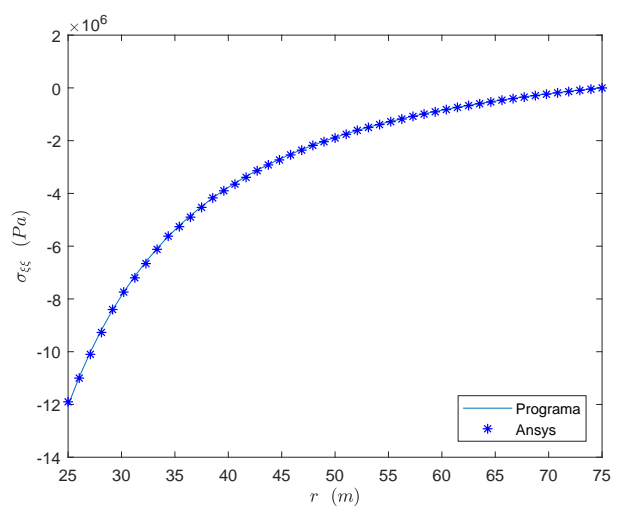

Figura 5.9: Tensão radial nó 1

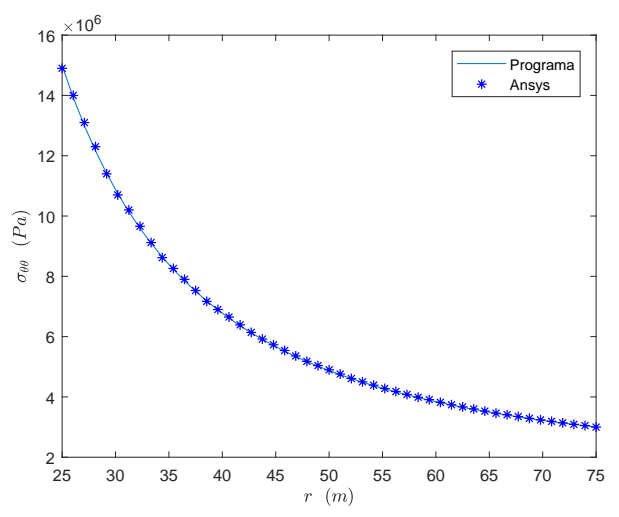

Figura 5.10: Tensão circunferencial nó 1 

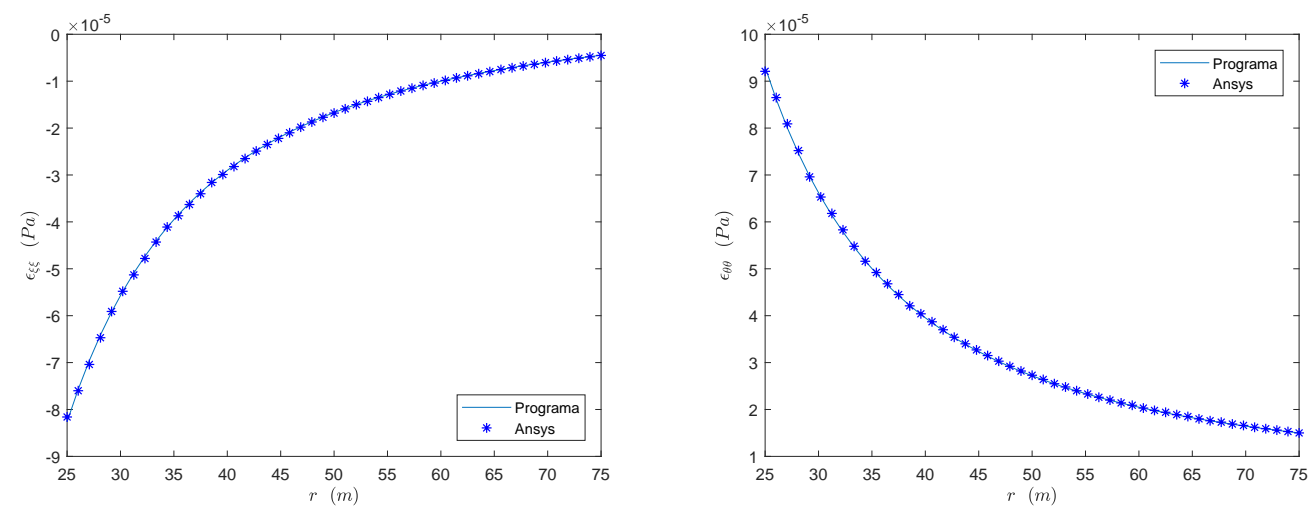

Figura 5.11: Deformação radial nó 1 Figura 5.12: Deformação circunferencial nó 1

\section{Cilindro livre sujeito a pressão externa de $12 \mathrm{MPa}$}

Assim como modelado no caso anterior, será considerado um vaso de pressão com condições de contorno livres, todavia, ao invés de pressão interna, se considerará pressão externa. Os resultados estão conforme o esperado, fato que se pode comprovar nos gráficos abaixos.

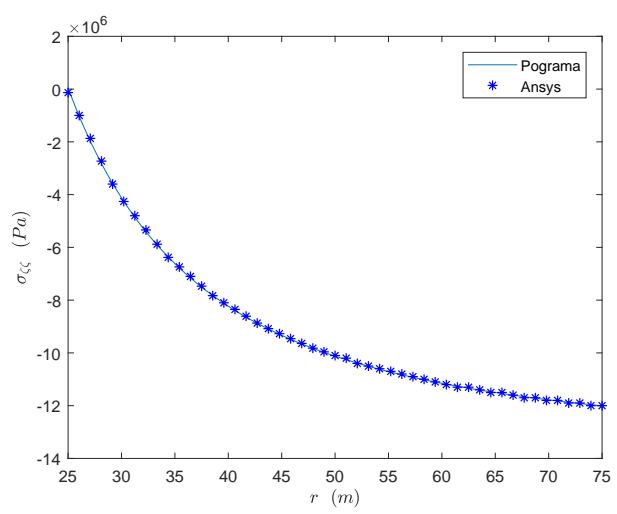

Figura 5.13: Tensão radial nó 1

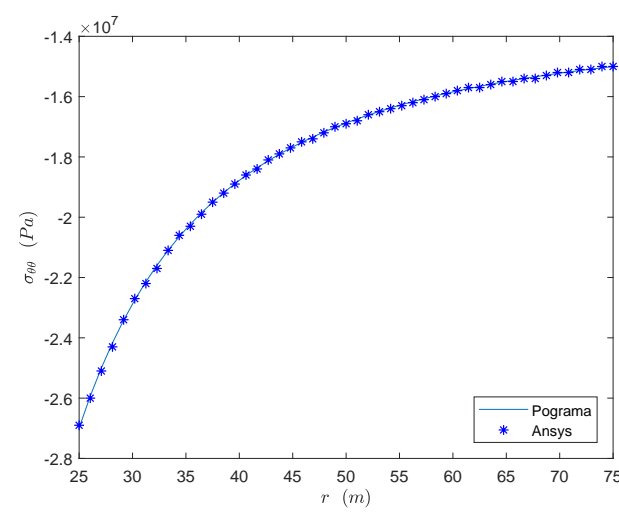

Figura 5.14: Tensão circunferencial nó 1 

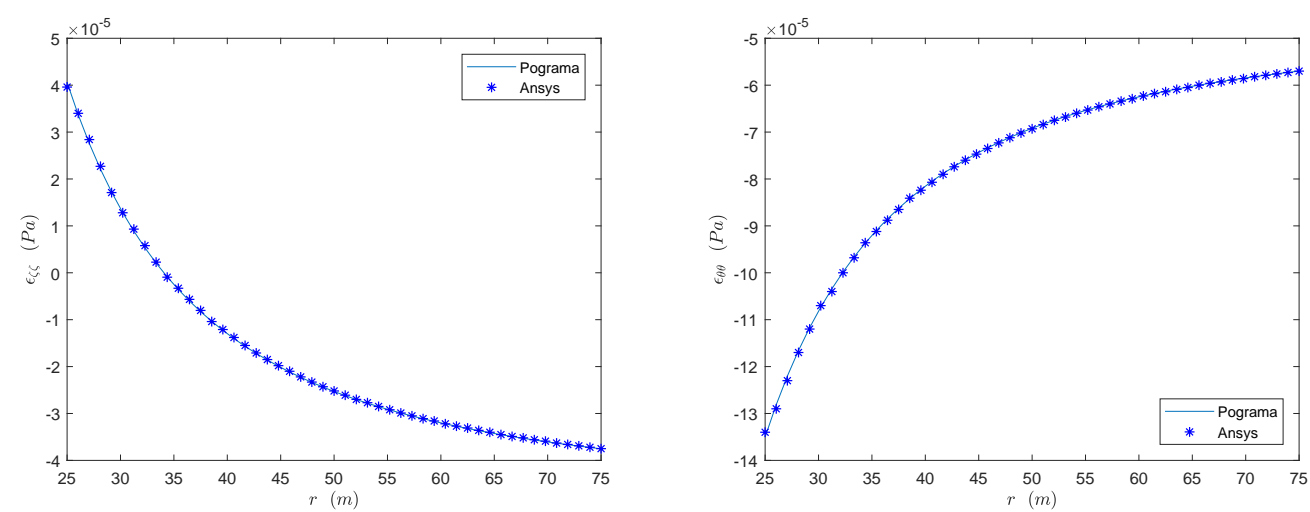

Figura 5.15: Deformação radial nó 1 Figura 5.16: Deformação circunferencial nó 1

\section{2}

\section{Cilindro sujeito à rotação}

Nesta seção, será estudado o efeito da rotação, com dados geométricos diferentes da seção anterior, listados abaixo

- Módulo de elasticidade: $200 \mathrm{GPa}$

- Limite de Escoamento: $285 \mathrm{MPa}$ Logo:

- Coeficiente de Poison: 0.3

- Raio interno: $2 \mathrm{~m}$

- Espessura: $1 \mathrm{~m}$

- Raio externo: $3 \mathrm{~m}$

- Raio médio: $2.5 \mathrm{~m}$

Todavia, para esta seção somente será estudado caso de cilindro livre como condição de contorno, implementado como:

\begin{tabular}{|c|c|c|c|}
\hline Nós & \multicolumn{3}{|c|}{ Condições de contorno } \\
\hline 1 & 0 & 0 & 0 \\
\hline 2 & 0 & 0 & 0 \\
\hline 3 & 0 & 0 & 0 \\
\hline 4 & 0 & 0 & 0 \\
\hline
\end{tabular}




\section{Cilindro livre sujeito à rotação de $30 \mathrm{rad} / \mathrm{s}$}

Diferentemente da seção anterior, será considerado nessa análise exclusivamente o efeito do carregamento de rotação a um vaso de pressão. Dessa maneira, mantendo-se como condição de contorno o vaso de pressão livre, vale a solução analítica. Assim, os gráficos a seguir apresentam a comparação dos resultados.

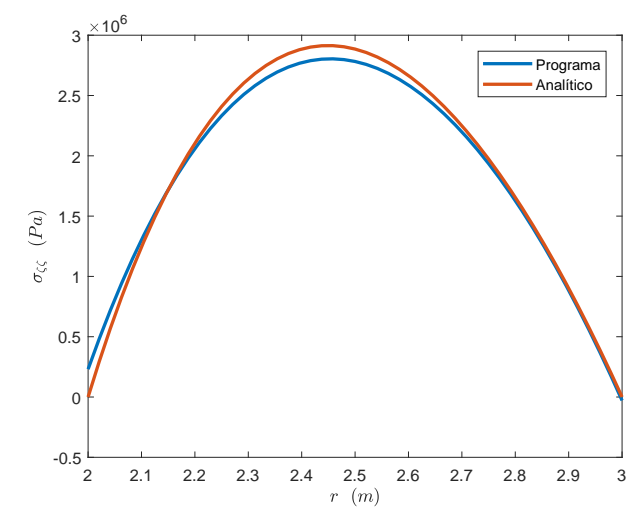

Figura 5.17: Tensão radial ao longo da Figura 5.18: Tensão circunferencial ao espessura no nó 1

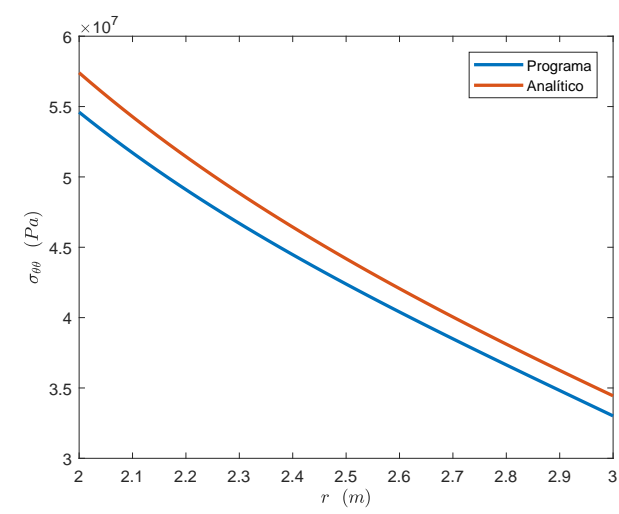

longo da espessura no nó 1

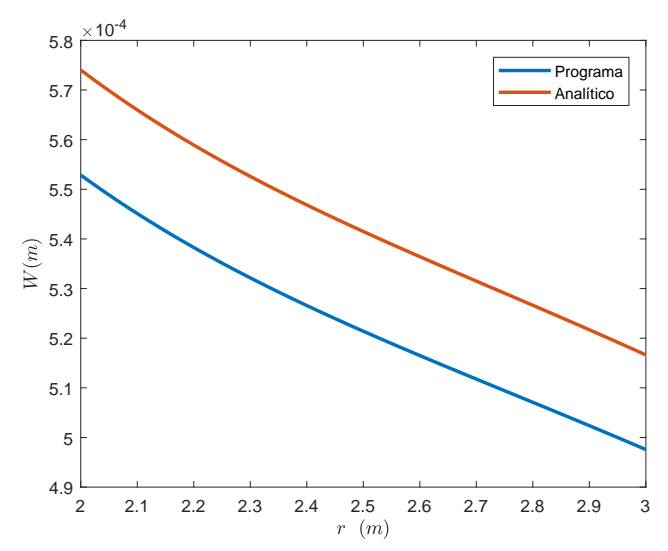

Figura 5.19: Deslocamento radial nó 1

Percebe-se que os três resultados apresentados acima estão conforme o esperado. Uma análise importante em estudos numéricos é a verificação do erro percentual entre os modelos comparados. Para o deslocamento radial, obtevese:

$$
\text { erro }_{W}=3.7 \%
$$

Já para as tensões radiais e circunferencias, o erro encontrado respectivamente foi:

$$
\operatorname{erro}_{\zeta}=3.6 \%
$$




$$
\operatorname{erro}_{\theta}=4.1 \%
$$

Os valores de erro acima foram calculados na casca média, e estão em conformidade com a metodologia utilizada, pois as deformações deselvolvidas na teoria de cascas valem para cascas finas ou semi-espessas. 


\section{Cilindro livre sujeito à rotação de $30 \mathrm{rad} / \mathrm{s}$ e pressão interna de $5 \mathrm{MPa}$}

O próximo caso a ser considerado é o efeito da rotação num vaso de pressão com pressão interna. Para isso, a fim de validarmos o desenvolvimento proposto no presente trabalho será comparado com o software comercial Ansys.

Conforme apresentado na seção "Resumo das soluções analíticas" no capítulo 1, não se pode superpor a solução do cilindro submetido à rotação com a solução do cilindro com carregamento de pressão interna, pois suas condições de contorno são distintas.

O gráfico 5.22 apresenta o deslocamento radial ao longo da espessura. Nota-se que os dois resultados são bastante próximos entre si. A tensão radial, gráfico 5.20, apresenta um comportamento esperado para um vaso, pois as tensões nas paredes (internas e externas) são nulas. No cálculo das tensões circunferenciais percebe-se, novamente, grande proximidade dos modelos usados.

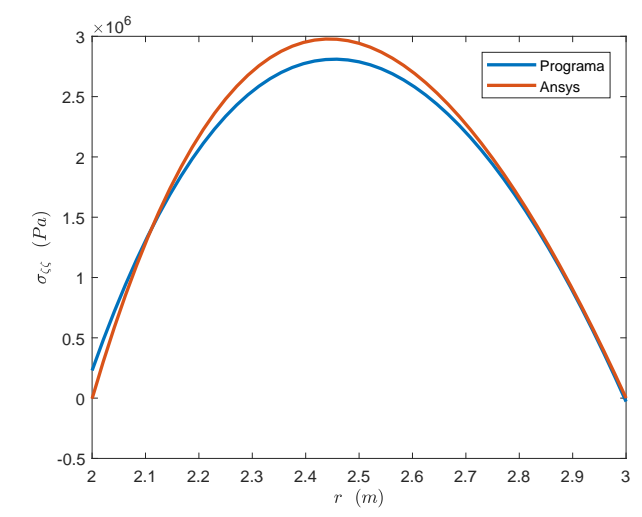

Figura 5.20: Tensão radial ao longo da Figura 5.21: Tensão circunferencial ao espessura no nó 1

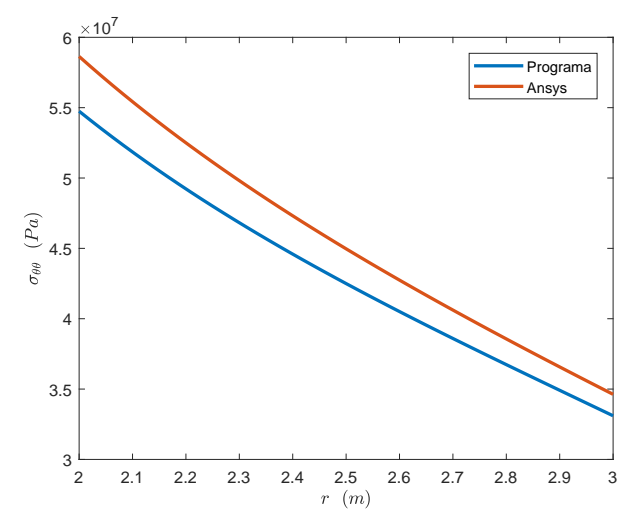

longo da espessura no nó 1

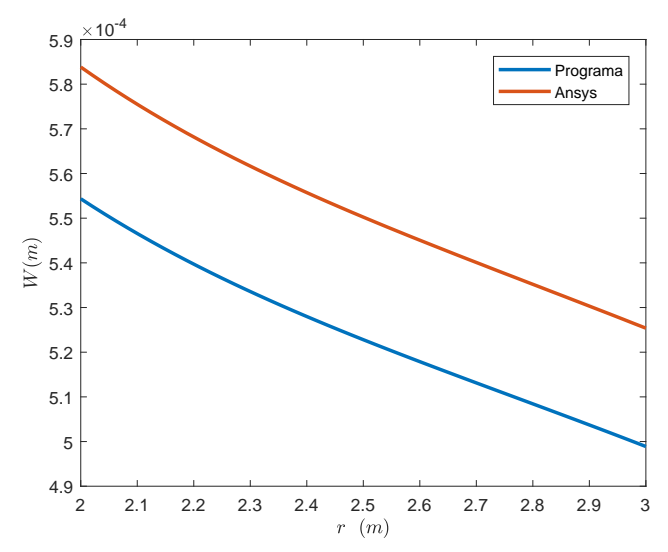

Figura 5.22: Deslocamento radial nó 1 
Uma análise importante em estudos numéricos é a verificação do erro percentual entre os modelos comparados. Para o deslocamento radial, obtevese:

$$
\text { erro }_{W}=4.9 \%
$$

Já para as tensões radiais e circunferencias, o erro encontrado respectivamente foi:

$$
\begin{aligned}
& \operatorname{erro}_{\zeta}=5.2 \% \\
& \text { erro }_{\theta}=5.5 \%
\end{aligned}
$$

Os valores de erro acima foram calculados na casca média e estão em conformidade com a metodologia utilizada, pois as deformações desenvolvidas na teoria de cascas valem para cascas finas ou semi-espessas. 


\section{Estudo do início da plasticidade em vasos de pressão com rotação}

Nesta seção, será estudado o início da plasticidade em vasos de pressão cilíndricos. A análise desenvolvida neste trabalho contempla apenas elementos finitos lineares, não incluindo o efeito da plasticidade do material. Assim, os resultados numéricos obtidos podem não condizer com a realidade, caso ultrapasse a resistência ao escoamento.

A fim de se determinar a velocidade limite de rotação para que não ocorra escoamento, um método iterativo foi desenvolvido, utilizando o critério de Von Mises para comparar tensões em um modelo axissimétrico com um uniaxial, conforme equacionado abaixo.

$$
\sigma_{\text {Mises }}=\sqrt{\frac{\left(\sigma_{\zeta \zeta}-\sigma_{\xi \xi}\right)^{2}+\left(\sigma_{\xi \xi}-\sigma_{\theta \theta}\right)^{2}+\left(\sigma_{\theta \theta}-\sigma_{\zeta \zeta}\right)^{2}+6 \cdot \tau_{\xi \zeta}^{2}}{2}}
$$

O resultado obtido a partir do método iterativo para a velocidade angular limite que garante o regime linear elástico é

$$
\omega_{p l, \text { programa }}=66.0 \mathrm{rad} / \mathrm{s}
$$

Para comparação, será estudado analiticamente o cálculo das tensões de Mises e velocidade angular que iniciam o escoamento. Sabendo que o cilindro é livre, sua tensão axial será zero e, como consequência, a tensão de Mises será calculada utilizando-se somente as equações analíticas de tensões radial e circunferencial. Pela figura 5.23, percebe-se que a maior diferença entre a tensão radial e circunferencial se dá na parede interno do vaso de pressão, local do início do escoamento.

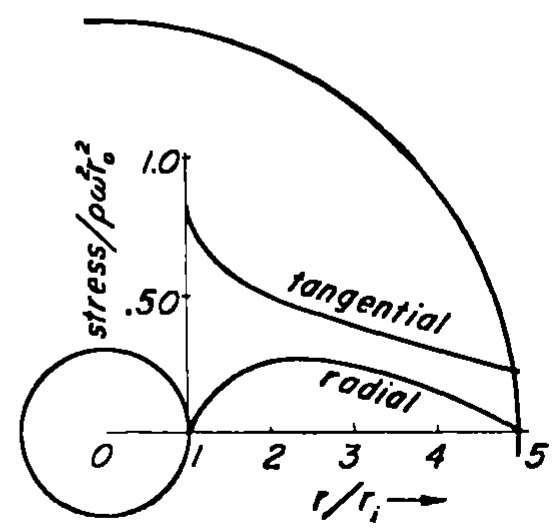

Figura 5.23: Distribuição de tensão para um disco girante

Pelas equações (1-87) e (1-88) tem-se 


$$
\begin{gathered}
\sigma_{r}\left(r_{i}\right)=0 \\
\sigma_{c}\left(r_{i}\right)=\frac{3+\nu}{4} \rho \omega^{2}\left(r_{o}^{2}+\frac{1-\nu}{3+\nu} r_{i}^{2}\right)
\end{gathered}
$$

Substituindo as equações $(5-9)$ e (5-10) em (5-7), resulta

$$
\omega_{p l}^{2}=\frac{1}{\rho} \cdot \frac{4 \cdot \sigma_{y p}}{(3+\nu) r_{o}^{2}+(1-\nu) r_{i}^{2}}
$$

Resolvendo a equação analítica acima, obtem-se

$$
\omega_{p l, \text { analitico }}=66.8 \mathrm{rad} / \mathrm{s}
$$

Com a velocidade angular analítica, é possível determinar a tensão de Mises ao longo da espessura utilizando as equações anlíticas das tensões. $\mathrm{O}$ gráfico 5.24 apresenta os resultados obtidos:

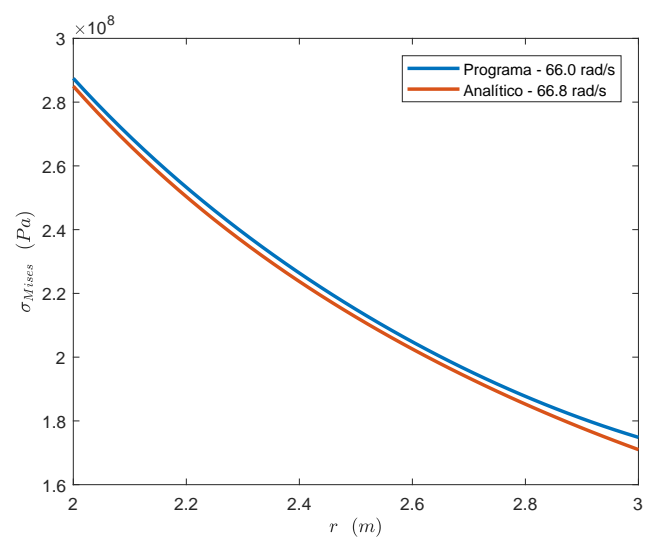

Figura 5.24: Tensão de Mises nó 1

Percebe-se que os resultados encontrados estão de acordo com o esperado, pois o escoamento ocorre na superfície interna do vaso de pressão cilíndrico e os valores de velocidade de rotação numérico e analítico estão bastante próximos.

O erro máximo percentual no gráfico foi de

$$
\text { erro }_{\max }=1.2 \%
$$

valor encontrado baixo, ratificando a metodologia utilizado de elementos finitos enriquecidos. 


\section{6 \\ Conclusão}

Analisando os resultados encontrados, percebe-se que o modelo apresentado no trabalho reproduz os casos esperados para vasos de pressão cilíndricos, quando comparados as soluções numéricas provindas do software Ansys e as soluções analíticas.

Nesse estudo foi simulada a verificação do programa, comparando seu resultado com a solução analítica de Lamé sob condições de contorno de travamento do deslocamento axial e rotação da seção reta na base do cilindro. Além disso, outro caso analisado foi o cilindro sob condição de contorno livre com carregamento de pressão. Em seguida, para cilindros livres foi considerado a rotação como carregamento de força de corpo. Os resultados encontrados foram comparados com o Ansys ou com soluções analíticas conhecidas da literatura. Pode-se concluir que os resultados obtidos estão bastante próximos dos esperados.

O último caso analisado foi o início da plasticidade em cilindros livres sob rotação. Ao se calcular a tensão de Mises ao longo da espessura, foi possível determinar o valor da velocidade angular capaz de começar o escoamento na parede interna do vaso de pressão. Ao se comparar o resultado numérico encontrado com o analítico da velocidade angular, o erro relativo encontrado foi muito baixo, demonstrando proximidade do método desenvolvido no trabalho.

Vale ressaltar que os casos estudados foram com o uso de apenas um elemento, fato importante para ratificar o emprego de elementos finitos enriquecidos. Todavia, possíveis diferenças entre as soluções analíticas e numéricas pelo Ansys com a solução elaborada estão previstas nas hipóteses de validade do modelo. Isso porque as equações das deformações provindas da teoria de cascas são restritas às cascas finas e semi-espessas. Contudo, ao se utilizar cilindros espessos, percebeu-se que o modelo, ainda assim, obteve resultado conforme o esperado.

Nota-se que o objetivo do trabalho foi alcançado ao apresentar uma metodologia numérica capaz de obter os resultados sem a necessidade de um maior número de elementos. 


\section{Apêndice}

\section{1}

\section{Apêndice - Fórmula 1}

$$
\begin{aligned}
(d s)^{2}=d \hat{X} \cdot d \hat{X}+2 z d \hat{X} \cdot d \hat{n}+2 d z(d \hat{X} \cdot \hat{n}) & \\
& +z^{2}(d \hat{n} \cdot d \hat{n})+2 z d z(\hat{n} \cdot d \hat{n})+(d z)^{2}(\hat{n} \cdot \hat{n})
\end{aligned}
$$

Pelas equações (2-9), 2-20), 2-38), 2-39), 2-46) e (2-47) têm-se:

$$
\begin{gathered}
d \hat{X} \cdot d \hat{X}=A_{1}^{2}(d \xi)^{2}+A_{2}^{2}(d \theta)^{2} \\
K_{n}=\frac{d \hat{n} \cdot \hat{X}}{d \hat{X} \cdot d \hat{X}} \quad \therefore \quad \hat{X}=K_{n} \cdot(d \hat{X} \cdot d \hat{X}) \\
d \hat{X} \cdot d \hat{n}=L(d \xi)^{2}+N(d \theta)^{2}=\frac{A_{1}^{2}}{R_{1}}(d \xi)^{2}+\frac{A_{2}^{2}}{R_{2}}(d \theta)^{2}
\end{gathered}
$$

Sabendo que $d \hat{X}$ é o vetor tangente a superfície e $\hat{n}$ o vetor normal, portanto:

$$
\begin{gathered}
d \hat{X} \cdot d \hat{n}=0 \\
d \hat{n} \cdot d \hat{n}=\left(\frac{A_{1}}{R_{1}}\right)^{2}(d \xi)^{2}+\left(\frac{A_{2}}{R_{2}}\right)^{2}(d \theta)^{2}
\end{gathered}
$$

Como o vetor $d \hat{n}$ é tangente a superfície e o vetor $\hat{n}$ normal, então seu produto interno será zero.

$$
d \hat{n} \cdot \hat{n}=0
$$

O produto interno com dois vetores unitário de iguais será 1, em particular, aplicando para o vetor normal $\hat{n}$ :

$$
\hat{n} \cdot \hat{n}=1
$$


Substituindo:

$$
\begin{aligned}
&(d s)^{2}=A_{1}^{2}(d \xi)^{2}+A_{2}^{2}(d \theta)^{2}+2 z\left(\frac{A_{1}^{2}}{R_{1}}\right)(d \xi)^{2} \\
&+2 z\left(\frac{A_{2}^{2}}{R_{2}}\right)(d \theta)^{2}+z^{2}\left(\frac{A_{2}^{2}}{R_{2}}\right)(d \theta)^{2}+z^{2}\left(\frac{A_{1}}{R_{1}}\right)^{2}(d \xi)^{2} \\
&+Z^{2}\left(\frac{A_{2}}{R_{2}}\right)^{2}(d \theta)^{2}+(d z)^{2}
\end{aligned}
$$

Finalmente:

$$
(d s)^{2}=A_{1}^{2}\left(1+\frac{z}{R_{1}}\right)^{2}(d \xi)^{2}+A_{2}^{2}\left(1+\frac{z}{R_{2}}\right)^{2}(d \theta)^{2}+(d z)^{2}
$$




\section{2}

\section{Apêndice - Condições de Contorno Naturais}

$$
\begin{gathered}
\left.\gamma_{\xi \zeta}\right|_{\zeta= \pm 1}=0 \\
\gamma_{\xi \zeta}=\frac{1}{s^{\prime}} \cdot \frac{\partial W}{\partial \xi}+\frac{\partial U_{1}}{\partial T}
\end{gathered}
$$

Portanto:

$$
\left.\gamma_{\xi \zeta}\right|_{\zeta= \pm 1}=\left[\frac{1}{s^{\prime}} \frac{\partial w}{\partial \xi}\right]_{\zeta= \pm 1}+\left[\frac{\partial U_{1}}{\partial T}\right]_{\zeta= \pm 1}
$$

Logo:

Sabendo que:

$$
\begin{aligned}
& \left.\frac{1}{s^{\prime}} \frac{\partial W}{\partial \xi}\right|_{\zeta=+1}+\left.\frac{\partial U_{1}}{\partial T}\right|_{\zeta=+1}=0 \\
& \left.\frac{1}{s^{\prime}} \frac{\partial W}{\partial \xi}\right|_{\zeta=-1}+\left.\frac{\partial U_{1}}{\partial T}\right|_{\zeta=-1}=0
\end{aligned}
$$

$$
U_{1}(\xi, \zeta)=U_{1}^{0}(\xi)+\gamma_{1}(\xi) \cdot T+\phi_{1}(\xi) \cdot T^{2}+\psi_{1}(\xi) \cdot T^{3}
$$

Substituindo a equação acima na equação número 7-5, tem-se:

$$
\begin{gathered}
\left.\frac{1}{s^{\prime}} \frac{\partial W}{\partial \xi}\right|_{\zeta=+1}+\frac{\partial}{\partial T}\left[U_{1}^{0}(\xi)+\gamma_{1}(\xi) \cdot T+\phi_{1}(\xi) \cdot T^{2}+\psi_{1}(\xi) \cdot T^{3}\right]_{\zeta=+1}=0 \\
\left.\frac{1}{s^{\prime}} \frac{\partial W}{\partial \xi}\right|_{\zeta=+1}+\frac{\partial}{\partial T}\left[\gamma_{1}(\xi)+2 \cdot \phi_{1}(\xi) \cdot T+3 \cdot \psi_{1}(\xi) \cdot T^{2}\right]_{\zeta=+1}=0
\end{gathered}
$$

Sabendo que:

$$
\begin{gathered}
T=\frac{h}{2} \zeta \\
\left.\frac{1}{s^{\prime}} \frac{\partial W}{\partial \xi}\right|_{\zeta=+1}+\frac{\partial}{\partial T}\left[\gamma_{1}(\xi)+2 \cdot \phi_{1}(\xi) \frac{h}{2} \zeta+3 \cdot \psi_{1}(\xi) \cdot T^{2}\right]_{\zeta=+1}=0
\end{gathered}
$$

Lembrando que $\zeta=+1$ :

$$
\left.\frac{1}{s^{\prime}} \frac{\partial W}{\partial \xi}\right|_{\zeta=+1}+\gamma_{1}(\xi)+\phi_{1}(\xi) \cdot h+3 \cdot \psi_{1}(\xi) \cdot \frac{h^{2}}{4}=0
$$

Substituindo a equação acima na equação número 7-6, tem-se:

$$
\left.\frac{1}{s^{\prime}} \frac{\partial W}{\partial \xi}\right|_{\zeta=-1}+\frac{\partial}{\partial T}\left[U_{1}^{0}(\xi)+\gamma_{1}(\xi) \cdot T+\phi_{1}(\xi) \cdot T^{2}+\psi_{1}(\xi) \cdot T^{3}\right]_{\zeta=-1}=0
$$




$$
\left.\frac{1}{s^{\prime}} \frac{\partial W}{\partial \xi}\right|_{\zeta=-1}+\frac{\partial}{\partial T}\left[\gamma_{1}(\xi)+2 \cdot \phi_{1}(\xi) \cdot T+3 \cdot \psi_{1}(\xi) \cdot T^{2}\right]_{\zeta=-1}=0
$$

Sabendo que:

$$
\begin{gathered}
T=\frac{h}{2} \zeta \\
\left.\frac{1}{s^{\prime}} \frac{\partial W}{\partial \xi}\right|_{\zeta=-1}+\gamma_{1}(\xi)-\phi_{1}(\xi) \cdot h+3 \cdot \psi_{1}(\xi) \cdot \frac{h^{2}}{4}=0
\end{gathered}
$$

Temos como sistema as equações $7-7$ e $7-8$, para encontrarmos as variáveis $\phi_{1}(\xi)$ e $\psi_{1}(\xi)$.

$$
\begin{aligned}
& \left.\frac{1}{s^{\prime}} \frac{\partial W}{\partial \xi}\right|_{\zeta=+1}+\gamma_{1}(\xi)+\phi_{1}(\xi) \cdot h+3 \cdot \psi_{1}(\xi) \cdot \frac{h^{2}}{4}=0 \\
& \left.\frac{1}{s^{\prime}} \frac{\partial W}{\partial \xi}\right|_{\zeta=-1}+\gamma_{1}(\xi)-\phi_{1}(\xi) \cdot h+3 \cdot \psi_{1}(\xi) \cdot \frac{h^{2}}{4}=0
\end{aligned}
$$

Multiplicando a segunda equação por -1 , temos como sistema:

$$
\begin{aligned}
& \left.\frac{1}{s^{\prime}} \frac{\partial W}{\partial \xi}\right|_{\zeta=+1}+\gamma_{1}(\xi)+\phi_{1}(\xi) \cdot h+3 \cdot \psi_{1}(\xi) \cdot \frac{h^{2}}{4}=0 \\
& -\left.\frac{1}{s^{\prime}} \frac{\partial W}{\partial \xi}\right|_{\zeta=-1}-\gamma_{1}(\xi)+\phi_{1}(\xi) \cdot h-3 \cdot \psi_{1}(\xi) \cdot \frac{h^{2}}{4}=0
\end{aligned}
$$

Somando as duas equações do sistema acima:

$$
\begin{aligned}
& \left.\frac{1}{s^{\prime}} \frac{\partial W}{\partial \xi}\right|_{\zeta=+1}-\left.\frac{1}{s^{\prime}} \frac{\partial W}{\partial \xi}\right|_{\zeta=-1}+2 \cdot \phi_{1} \cdot h=0 \\
& \left.\frac{1}{s^{\prime}} \frac{\partial W}{\partial \xi}\right|_{\zeta=+1}-\left.\frac{1}{s^{\prime}} \frac{\partial W}{\partial \xi}\right|_{\zeta=-1}+=-2 \cdot \phi_{1} \cdot h
\end{aligned}
$$

Portanto:

$$
\phi_{1}(\xi)=-\left(\left.\frac{1}{s^{\prime}} \frac{\partial W}{\partial \xi}\right|_{\zeta=+1}-\left.\frac{1}{s^{\prime}} \frac{\partial W}{\partial \xi}\right|_{\zeta=-1}\right) \cdot \frac{1}{2 h}
$$

Sistema Original, referente as equações 7-7 e 7-8.

$$
\begin{aligned}
& \left.\frac{1}{s^{\prime}} \frac{\partial W}{\partial \xi}\right|_{\zeta=+1}+\gamma_{1}(\xi)+\phi_{1}(\xi) \cdot h+3 \cdot \psi_{1}(\xi) \cdot \frac{h^{2}}{4}=0 \\
& \left.\frac{1}{s^{\prime}} \frac{\partial W}{\partial \xi}\right|_{\zeta=-1}+\gamma_{1}(\xi)-\phi_{1}(\xi) \cdot h+3 \cdot \psi_{1}(\xi) \cdot \frac{h^{2}}{4}=0
\end{aligned}
$$


Somando as duas equações:

$$
\begin{gathered}
\left(\left.\frac{1}{s^{\prime}} \frac{\partial W}{\partial \xi}\right|_{\zeta=+1}+\left.\frac{1}{s^{\prime}} \frac{\partial W}{\partial \xi}\right|_{\zeta=-1}\right)+2 \cdot \gamma_{1}+6 \cdot \psi_{1} \cdot \frac{h^{2}}{4}=0 \\
-\frac{3 h^{2}}{2} \psi_{1}=\left(\left.\frac{1}{s^{\prime}} \frac{\partial W}{\partial \xi}\right|_{\zeta=+1}+\left.\frac{1}{s^{\prime}} \frac{\partial W}{\partial \xi}\right|_{\zeta=-1}\right)+2 \cdot \gamma_{1} \\
\psi_{1}(\xi)=-\left(\left.\frac{1}{s^{\prime}} \frac{\partial W}{\partial \xi}\right|_{\zeta=+1}+\left.\frac{1}{s^{\prime}} \frac{\partial W}{\partial \xi}\right|_{\zeta=-1}\right) \cdot \frac{2}{3 h^{2} s^{\prime}}-\frac{4 \gamma_{1}}{3 h^{2}}
\end{gathered}
$$


7.3

Apêndice - Matriz C

$$
\left[\begin{array}{c}
\sigma_{\xi \xi} \\
\sigma_{\theta \theta} \\
\sigma_{\zeta \zeta} \\
\tau_{\xi \zeta}
\end{array}\right]^{(n)}=\underline{C^{(n)}}\left[\begin{array}{c}
\epsilon_{\xi \xi} \\
\epsilon_{\theta \theta} \\
\epsilon_{\zeta \zeta} \\
\gamma_{\xi \zeta}
\end{array}\right]^{(n)}
$$

Equações Constitutivas:

$$
\begin{gathered}
\epsilon_{\xi \xi}=\frac{\sigma_{\xi \xi}}{E}-\nu \frac{\sigma_{\theta \theta}}{E}-\nu \frac{\sigma_{\zeta \zeta}}{E} \\
\epsilon_{\theta \theta}=\frac{\sigma_{\theta \theta}}{E}-\nu \frac{\sigma_{\xi \xi}}{E}-\nu \frac{\sigma_{\zeta \zeta}}{E} \\
\epsilon_{\zeta \zeta}=\frac{\sigma_{\zeta \zeta}}{E}-\nu \frac{\sigma_{\xi \xi}}{E}-\nu \frac{\sigma_{\theta \theta}}{E} \\
\gamma_{\xi \zeta}=\frac{E}{2(1+\nu)} \sigma_{\xi \zeta}
\end{gathered}
$$

Das equações acima temos:

$$
\begin{gathered}
\sigma_{\xi \xi}=\epsilon_{\xi \xi} E+\nu \sigma_{\theta \theta}+\nu \sigma_{\zeta \zeta} \\
\sigma_{\theta \theta}=\epsilon_{\theta \theta} E+\nu \sigma_{\xi \xi}+\nu \sigma_{\zeta \zeta} \\
\sigma_{\zeta \zeta}=\epsilon_{\zeta \zeta} E+\nu \sigma_{\xi \xi}+\nu \sigma_{\theta \theta}
\end{gathered}
$$

Substituindo 7-14 e 7-15 em 7-13, temos:

$$
\begin{gathered}
\sigma_{\xi \xi}=\epsilon_{\xi \xi} E+\nu\left[\epsilon_{\theta \theta} E+\nu \sigma_{\xi \xi}+\nu \sigma_{\zeta \zeta}\right]+\nu\left[\epsilon_{\zeta \zeta} E+\nu \sigma_{\xi \xi}+\nu \sigma_{\theta \theta}\right] \\
\sigma_{\xi \xi}=\epsilon_{\xi \xi} E+\nu \epsilon_{\theta \theta} E+\nu \epsilon_{\zeta \zeta} E+\nu^{2} \sigma_{\xi \xi}+\nu^{2} \sigma_{\zeta \zeta}+\nu^{2} \sigma_{\xi \xi}+\nu^{2} \sigma_{\theta \theta} \\
\sigma_{\xi \xi}=\epsilon_{\xi \xi} E+\nu \epsilon_{\theta \theta} E+\nu \epsilon_{\zeta \zeta} E+2 \nu^{2} \sigma_{\xi \xi}+\nu^{2} \sigma_{\theta \theta}+\nu^{2} \sigma_{\xi \xi} \\
\sigma_{\xi \xi}=\epsilon_{\xi \xi} E+\nu \epsilon_{\theta \theta} E+\nu \epsilon_{\zeta \zeta} E+2 \nu^{2} \sigma_{\xi \xi}+\nu\left(\nu \sigma_{\theta \theta}+\nu \sigma_{\zeta \zeta}\right)
\end{gathered}
$$

De 7-13, temos:

$$
\sigma_{\xi \xi}-\epsilon_{\xi \xi} E=\nu \sigma_{\theta \theta}+\nu \sigma_{\zeta \zeta}
$$


Portanto:

$$
\begin{gathered}
\sigma_{\xi \xi}=\epsilon_{\xi \xi} E+\nu \epsilon_{\theta \theta} E+\nu \epsilon_{\zeta \zeta} E+2 \nu^{2} \sigma_{\xi \xi}+\nu\left(\sigma_{\xi \xi}-\epsilon_{\xi \xi} E\right) \\
\sigma_{\xi \xi}=\epsilon_{\xi \xi} E+\nu \epsilon_{\theta \theta} E+\nu \epsilon_{\zeta \zeta} E+2 \nu^{2} \sigma_{\xi \xi}+\nu \sigma_{\xi \xi}-\nu \epsilon_{\xi \xi} E \\
\sigma_{\xi \xi}-\nu \sigma_{\xi \xi}-2 \nu^{2} \sigma_{\xi \xi}=\epsilon_{\xi \xi} E-\nu \epsilon_{\xi \xi} E+\nu \epsilon_{\theta \theta} E+\nu \epsilon_{\zeta \zeta} E \\
\left(1-\nu 2 \nu^{2}\right) \sigma_{\xi \xi}=\epsilon_{\xi \xi} E(1-\nu)+\nu \epsilon_{\theta \theta} E+\nu \epsilon_{\zeta \zeta} E \\
(1+\nu) \cdot(1-2 \nu) \sigma_{\xi \xi}=\epsilon_{\xi \xi} E(1-\nu)+\nu \epsilon_{\theta \theta} E+\nu \epsilon_{\zeta \zeta} E
\end{gathered}
$$

Logo:

$$
\sigma_{\xi \xi}=\frac{E}{(1+\nu) \cdot(1-2 \nu)}\left[(1-\nu) \epsilon_{\xi \xi}+\nu \epsilon_{\theta \theta}+\nu \epsilon_{\zeta \zeta}\right]
$$

Fazendo o procedimento acima para $\sigma_{\theta \theta}$ e $\sigma_{\zeta \zeta}$. Vale observar que devido a semelhança das equações 7-13, 7-14 e 7-15 não é necessário provar novamente, basta reescrever conforme a equação 7-16.

Assim:

$$
\begin{aligned}
\sigma_{\theta \theta} & =\frac{E}{(1+\nu) \cdot(1-2 \nu)}\left[(1-\nu) \epsilon_{\theta \theta}+\nu \epsilon_{\xi \xi}+\nu \epsilon_{\zeta \zeta}\right] \\
\sigma_{\zeta \zeta} & =\frac{E}{(1+\nu) \cdot(1-2 \nu)}\left[(1-\nu) \epsilon_{\zeta \zeta}+\nu \epsilon_{\xi \xi}+\nu \epsilon_{\theta \theta}\right]
\end{aligned}
$$

Assim:

$$
\left[\begin{array}{c}
\sigma_{\xi \xi} \\
\sigma_{\theta \theta} \\
\sigma_{\zeta \zeta} \\
\tau_{\xi \zeta}
\end{array}\right]^{(n)}=\frac{E}{(1+\nu) \cdot(1-2 \nu)} \cdot\left[\begin{array}{cccc}
(1-\nu) & \nu & \nu & 0 \\
\nu & (1-\nu) & \nu & 0 \\
\nu & \nu & (1-\nu) & 0 \\
0 & 0 & 0 & \frac{(1-2 \nu)}{2}
\end{array}\right]\left[\begin{array}{c}
\epsilon_{\xi \xi} \\
\epsilon_{\theta \theta} \\
\epsilon_{\zeta \zeta}^{(n)} \\
\gamma_{\xi \zeta}
\end{array}\right]^{(1-\nu}
$$

A matriz $C^{(n)}$ é dada portanto por:

$$
C^{(n)}=\frac{E}{(1+\nu) \cdot(1-2 \nu)} \cdot\left[\begin{array}{cccc}
(1-\nu) & \nu & \nu & 0 \\
\nu & (1-\nu) & \nu & 0 \\
\nu & \nu & (1-\nu) & 0 \\
0 & 0 & 0 & \frac{(1-2 \nu)}{2}
\end{array}\right]
$$




\section{4}

\section{Apêndice - Matriz B}

$$
\begin{array}{r}
\left.\frac{\partial W}{\partial \xi}\right|_{\zeta= \pm 1}=\frac{\partial}{\partial \xi}\left[\sum_{i=1}^{k} h_{i}(\xi) W_{0}^{i}+\frac{1}{R} \cdot \sum_{i=1}^{k} h_{i}(\xi) W_{1}^{i}+R \cdot \sum_{i=1}^{k} h_{i}(\xi) W_{2}^{i}+\right. \\
\left.\frac{1}{R^{2}} \cdot \sum_{i=1}^{k} h_{i}(\xi) W_{3}^{i}+R^{3} \cdot \sum_{i=1}^{k} h_{i}(\xi) W_{4}^{i}\right]_{\zeta= \pm 1}
\end{array}
$$

Onde:

$$
\begin{gathered}
R(\xi, \zeta)=R_{A}+T \\
T=\frac{h}{2} \cdot \zeta
\end{gathered}
$$

Logo:

$$
R(\xi, \zeta)=R_{A}+\frac{h}{2} \cdot \zeta
$$

Percebe-se, portanto que o primeiro termo da parcela da equação 7-17. $\sum_{i=1}^{k} h_{i}(\xi) w_{0}^{i}$ não depende de $\zeta$.

$$
\begin{aligned}
& W_{0}(\xi)=\sum_{i=1}^{k} h_{i}(\xi) W_{0}^{i} \\
& W_{1}(\xi)=\sum_{i=1}^{k} h_{i}(\xi) W_{1}^{i} \\
& W_{2}(\xi)=\sum_{i=1}^{k} h_{i}(\xi) W_{2}^{i} \\
& W_{3}(\xi)=\sum_{i=1}^{k} h_{i}(\xi) W_{3}^{i} \\
& W_{4}(\xi)=\sum_{i=1}^{k} h_{i}(\xi) W_{4}^{i}
\end{aligned}
$$

\subsection{1}

Deformação Longitudinal, $\epsilon_{\xi \xi}$

$$
\epsilon_{\xi \xi}=\frac{1}{s^{\prime}}\left(\frac{\partial U_{1}}{\partial \xi}\right)
$$

Onde:

$$
U_{1}(\xi, \zeta)=U_{1}^{0}(\xi)+\gamma_{1}(\xi) \cdot T+\phi_{1}(\xi) \cdot T^{2}+\psi_{1}(\xi) \cdot T^{3}
$$

Logo:

$$
\epsilon_{\xi \xi}=\frac{1}{s^{\prime}} \frac{\partial}{\partial \xi}\left(U_{0}(\xi)+\gamma_{1}(\xi) \cdot T+\phi_{1}(\xi) \cdot T^{2}+\psi_{1}(\xi) \cdot T^{3}\right)
$$




$$
\epsilon_{\xi \xi}=\frac{1}{s^{\prime}}\left(\frac{\partial}{\partial \xi}\left[\sum_{i=1}^{k} h_{i}(\xi) U_{0}^{i}\right]+\frac{\partial}{\partial \xi}\left[\sum_{i=1}^{k} h_{i}(\xi) \gamma_{1}^{i}\right] \cdot T+\frac{\partial}{\partial \xi}\left(\phi_{1}\right) T^{2}+\frac{\partial}{\partial \xi}\left(\psi_{1}\right) T^{3}\right)
$$

Substituindo na equação anterior as equações 7-9 e 7-10, onde:

$$
\begin{gathered}
\phi_{1}(\xi)=-\left(\left.\frac{1}{s^{\prime}} \frac{\partial W}{\partial \xi}\right|_{\zeta=+1}-\left.\frac{1}{s^{\prime}} \frac{\partial W}{\partial \xi}\right|_{\zeta=-1}\right) \cdot \frac{1}{2 h} \\
\psi_{1}(\xi)=-\left(\left.\frac{1}{s^{\prime}} \frac{\partial W}{\partial \xi}\right|_{\zeta=+1}+\left.\frac{1}{s^{\prime}} \frac{\partial W}{\partial \xi}\right|_{\zeta=-1}\right) \cdot \frac{2}{3 h^{2} s^{\prime}}-\frac{4 \gamma_{1}}{3 h^{2}}
\end{gathered}
$$

Portanto:

$$
\begin{aligned}
\epsilon_{\xi \xi}=\frac{1}{s^{\prime}}\left(\frac{\partial}{\partial \xi}\left[\sum_{i=1}^{k} h_{i}(\xi) U_{0}^{i}\right]+\frac{\partial}{\partial \xi}\left[\sum_{i=1}^{k} h_{i}(\xi) \gamma_{1}^{i}\right] \cdot T\right. \\
+\frac{\partial}{\partial \xi}\left(-\left(\left.\frac{1}{s^{\prime}} \frac{\partial W}{\partial \xi}\right|_{\zeta=+1}-\left.\frac{1}{s^{\prime}} \frac{\partial W}{\partial \xi}\right|_{\zeta=-1}\right) \cdot \frac{1}{2 h}\right) \cdot T^{2} \\
\left.+\frac{\partial}{\partial \xi}\left(-\left(\left.\frac{1}{s^{\prime}} \frac{\partial W}{\partial \xi}\right|_{\zeta=+1}+\left.\frac{1}{s^{\prime}} \frac{\partial W}{\partial \xi}\right|_{\zeta=-1}\right) \cdot \frac{2}{3 h^{2} s^{\prime}}-\frac{4 \gamma_{1}}{3 h^{2}}\right) T^{3}\right)
\end{aligned}
$$

O termo $\gamma_{1}$ deve ser expresso como uma série em função de cada $\gamma_{1}^{i}$, portanto:

$$
\begin{aligned}
\epsilon_{\xi \xi}=\frac{1}{s^{\prime}}\left(\frac{\partial}{\partial \xi}\left[\sum_{i=1}^{k} h_{i}(\xi) U_{0}^{i}\right]+\frac{\partial}{\partial \xi}\left[\sum_{i=1}^{k} h_{i}(\xi) \gamma_{1}^{i}\right] \cdot T\right. \\
+\frac{\partial}{\partial \xi}\left(-\left(\left.\frac{1}{s^{\prime}} \frac{\partial W}{\partial \xi}\right|_{\zeta=+1}-\left.\frac{1}{s^{\prime}} \frac{\partial W}{\partial \xi}\right|_{\zeta=-1}\right) \cdot \frac{1}{2 h}\right) \cdot T^{2} \\
\left.+\frac{\partial}{\partial \xi}\left(-\left(\left.\frac{1}{s^{\prime}} \frac{\partial W}{\partial \xi}\right|_{\zeta=+1}+\left.\frac{1}{s^{\prime}} \frac{\partial W}{\partial \xi}\right|_{\zeta=-1}\right) \cdot \frac{2}{3 h^{2} s^{\prime}}-\frac{4 \sum_{i=1}^{k} h_{i}(\xi) \gamma_{1}^{i}}{3 h^{2}}\right) T^{3}\right)
\end{aligned}
$$

- Isolar $U_{o}^{i}$

$$
a_{11}=\frac{1}{s^{\prime}} \frac{\partial h_{i}}{\partial \xi}
$$

- Isolar $\gamma_{1}^{i}$

$$
a_{12}=\frac{1}{s^{\prime}}\left[\frac{\partial h_{i}}{\partial \xi} \cdot T+\frac{\partial}{\partial \xi}\left(-\frac{4 h_{i}}{3 h^{2}}\right) \cdot T^{3}\right]
$$

Substituindo T pela equação $7-18$.

$$
a_{12}=\frac{1}{s^{\prime}}\left[\frac{\partial h_{i}}{\partial \xi} \cdot \frac{h}{2} \zeta-\frac{4}{3 h^{2}} \frac{\partial h_{i}}{\partial \xi} \cdot \frac{h^{3}}{8} \zeta^{3}\right]
$$


Portanto:

$$
a_{12}=\frac{1}{s^{\prime}} \frac{\partial h_{i}}{\partial \xi}\left(\frac{h}{2} \zeta-\frac{h}{6} \zeta^{3}\right)
$$

- Isolar $W_{0}^{i}$

$$
\begin{gathered}
a_{13}=\frac{1}{s^{\prime}}\left[\frac{\partial}{\partial \xi}\left(-\left(\frac{1}{s^{\prime}} \frac{\partial h_{i}}{\partial \xi}-\frac{1}{s^{\prime}} \frac{\partial h_{i}}{\partial \xi}\right) \cdot \frac{1}{2 h}\right) \cdot T^{2}+\frac{\partial}{\partial \xi}\left(-\left(\frac{\partial h_{i}}{\partial \xi}+\frac{\partial h_{i}}{\partial \xi}\right) \cdot \frac{2}{3 h^{2} s^{\prime}}\right) \cdot T^{3}\right] \\
a_{13}=\frac{1}{s^{\prime}}\left[\frac{\partial}{\partial \xi}\left(-(0) \cdot \frac{1}{2 h}\right) \cdot T^{2}+\frac{\partial}{\partial \xi}\left(-\left(2 \cdot \frac{\partial h_{i}}{\partial \xi}\right) \cdot \frac{2}{3 h^{2} s^{\prime}}\right) \cdot T^{3}\right] \\
a_{13}=\frac{1}{s^{\prime}} \frac{\partial^{2} h_{i}}{\partial \xi^{2}}(-2) \frac{2}{3 h^{2} s^{\prime}} \frac{h^{3}}{8} \zeta^{3}
\end{gathered}
$$

Portanto:

$$
a_{13}=-\frac{h \zeta^{3}}{6 s^{\prime 2}} \frac{\partial^{2} h_{i}}{\partial \xi^{2}}
$$

- Isolar $W_{1}^{i}$

$$
\begin{aligned}
& a_{14}=\frac{1}{s^{\prime}}\left[\frac{\partial}{\partial \xi}\left(-\left(\frac{1}{s^{\prime}} \frac{\partial}{\partial \xi}\left(\frac{h_{i}}{\left.R\right|_{\zeta=+1}}\right)-\frac{1}{s^{\prime}} \frac{\partial}{\partial \xi}\left(\frac{h_{i}}{\left.R\right|_{\zeta=-1}}\right)\right) \cdot \frac{1}{2 h}\right)\right) \cdot T^{2}+ \\
& \left.\frac{\partial}{\partial \xi}\left(-\left(\frac{\partial}{\partial \xi}\left(\frac{h_{i}}{\left.R\right|_{\zeta=+1}}\right)+\frac{\partial}{\partial \xi}\left(\frac{h_{i}}{\left.R\right|_{\zeta=-1}}\right)\right) \cdot \frac{2}{3 h^{2} s^{\prime}}\right) \cdot T^{3}\right] \\
& a_{14}=\frac{1}{s^{\prime}}\left[\frac{\partial}{\partial \xi}\left(-\frac{1}{s^{\prime}} \frac{\partial h_{i}}{\partial \xi}\left(\frac{1}{R_{0}}-\frac{1}{R_{i}}\right) \cdot \frac{1}{2 h}\right) \cdot T^{2}\right. \\
& \left.-\frac{\partial}{\partial \xi}\left(\frac{\partial h_{i}}{\partial \xi}\left(\frac{1}{R_{0}}+\frac{1}{R_{i}}\right) \cdot \frac{2}{3 h^{2} s^{\prime}}\right) \cdot T^{3}\right] \\
& a_{14}=\frac{1}{s^{\prime}}\left[-\frac{1}{s^{\prime}}\left(\frac{1}{R_{0}}-\frac{1}{R_{i}}\right) \frac{\partial^{2} h_{i}}{\partial \xi^{2}} \cdot \frac{1}{2 h} \cdot T^{2}-\frac{1}{s^{\prime}}\left(\frac{1}{R_{0}}+\frac{1}{R_{i}}\right) \frac{\partial^{2} h_{i}}{\partial \xi^{2}} \frac{2}{3 h^{2}} \cdot T^{3}\right]
\end{aligned}
$$

Substituindo $T$ pela equação 7-18, temos:

$$
a_{14}=\frac{1}{s^{\prime}}\left[-\frac{1}{s^{\prime}}\left(\frac{1}{R_{0}}-\frac{1}{R_{i}}\right) \frac{\partial^{2} h_{i}}{\partial \xi^{2}} \cdot \frac{1}{2 h} \cdot \frac{h^{2}}{4} \zeta^{2}-\frac{1}{s^{\prime}}\left(\frac{1}{R_{0}}+\frac{1}{R_{i}}\right) \frac{\partial^{2} h_{i}}{\partial \xi^{2}} \frac{2}{3 h^{2}} \cdot \frac{h^{3}}{8} \zeta^{3}\right]
$$

Simplificando:

$$
a_{14}=-\frac{1}{s^{\prime 2}} \frac{\partial^{2} h_{i}}{\partial \xi^{2}}\left[\left(\frac{1}{R_{0}}-\frac{1}{R_{i}}\right) \frac{h \zeta^{2}}{8}+\left(\frac{1}{R_{0}}+\frac{1}{R_{i}}\right) \frac{h \zeta^{3}}{12}\right]
$$




$$
a_{14}=-\frac{1}{s^{\prime 2}} \frac{\partial^{2} h_{i}}{\partial \xi^{2}} \frac{h \zeta^{2}}{4}\left[\left(\frac{1}{R_{0}}-\frac{1}{R_{i}}\right) \frac{1}{2}+\left(\frac{1}{R_{0}}+\frac{1}{R_{i}}\right) \frac{\zeta}{3}\right]
$$

Portanto:

$$
a_{14}=-\frac{h \zeta^{2}}{4 s^{\prime 2}} \frac{\partial^{2} h_{i}}{\partial \xi^{2}}\left[\left(\frac{1}{R_{0}}-\frac{1}{R_{i}}\right) \frac{1}{2}+\left(\frac{1}{R_{0}}+\frac{1}{R_{i}}\right) \frac{\zeta}{3}\right]
$$

- Isolar $W_{2}^{i}$

$$
\begin{aligned}
a_{15}=\frac{1}{s^{\prime}}\left[\frac{\partial}{\partial \xi}\left(-\left(\frac{1}{s^{\prime}} \frac{\partial}{\partial \xi}\left(\left.R\right|_{\zeta=+1} h_{i}\right)-\frac{1}{s^{\prime}} \frac{\partial}{\partial \xi}\left(\left.R\right|_{\zeta=-1} h_{i}\right)\right) \frac{1}{2 h}\right) \cdot T^{2}\right. \\
\left.+\frac{\partial}{\partial \xi}\left(-\left(\frac{\partial}{\partial \xi}\left(\left.R\right|_{\zeta=+1} h_{i}\right)+\frac{\partial}{\partial \xi}\left(\left.R\right|_{\zeta=-1} h_{i}\right)\right) \frac{2}{3 h^{2} s^{\prime}}\right) \cdot T^{3}\right] \\
a_{15}=\frac{1}{s^{\prime}}\left[-\frac{1}{s^{\prime}} \frac{\partial^{2} h_{i}}{\partial \xi^{2}}\left(R_{0}-R_{i}\right) \frac{1}{2 h} T^{2}-\frac{\partial}{\partial \xi}\left(\frac{\partial h_{i}}{\partial \xi}\left(R_{0}+R_{i}\right) \frac{2}{3 h^{2} s^{\prime}}\right) \cdot T^{3}\right] \\
a_{15}=-\frac{1}{s^{\prime}}\left[\frac{1}{s^{\prime}} \frac{\partial^{2} h_{i}}{\partial \xi^{2}}\left(R_{0}-R_{i}\right) \frac{1}{2 h} \frac{h^{2}}{4} \zeta^{2}+\frac{1}{s^{\prime}} \frac{\partial^{2} h_{i}}{\partial \xi^{2}}\left(R_{0}+R_{i}\right) \frac{2}{3 h^{2}} \frac{h^{3}}{8} \zeta^{3}\right] \\
a_{15}=-\frac{1}{s^{\prime 2}} \frac{\partial^{2} h_{i}}{\partial \xi^{2}}\left[\left(R_{0}-R_{i}\right) \cdot \frac{\zeta^{2}}{8}+\left(R_{0}+R_{i}\right) \cdot \frac{\zeta^{3}}{12}\right] \\
a_{15}=-\frac{1}{s^{\prime 2}} \frac{\partial^{2} h_{i}}{\partial \xi^{2}} \frac{\zeta^{2}}{4}\left[\left(R_{0}-R_{i}\right) \cdot \frac{1}{2}+\left(R_{0}+R_{i}\right) \cdot \frac{\zeta}{3}\right]
\end{aligned}
$$

Sabendo que:

$$
\begin{gathered}
\left(R_{0}-R_{i}\right)=h \\
\left(R_{0}+R_{i}\right)=\left(R_{2}+h+R_{2}-h\right)=2 \cdot R_{2}
\end{gathered}
$$

Portanto:

$$
a_{15}=-\frac{1}{s^{2}} \frac{\partial^{2} h_{i}}{\partial \xi^{2}} \frac{\zeta^{2}}{4}\left[\frac{h}{2}+\frac{2 \cdot R_{2}}{3} \zeta\right]
$$


- Isolar $W_{3}^{i}$

$$
\begin{aligned}
& a_{16}=\frac{1}{s^{\prime}}[ \frac{\partial}{\partial \xi}\left(-\left(\frac{1}{s^{\prime}} \frac{\partial}{\partial \xi}\left(\frac{h_{i}}{\left.R^{2}\right|_{\zeta=+1}}\right)-\frac{1}{s^{\prime}} \frac{\partial}{\partial \xi}\left(\frac{h_{i}}{\left.R^{2}\right|_{\zeta=-1}}\right)\right) \frac{1}{2 h}\right) \cdot T^{2} \\
&\left.+\frac{\partial}{\partial \xi}\left(-\left(\frac{\partial}{\partial \xi}\left(\frac{h_{i}}{\left.R^{2}\right|_{\zeta=+1}}\right)+\frac{\partial}{\partial \xi}\left(\frac{h_{i}}{\left.R^{2}\right|_{\zeta=-1}}\right)\right) \frac{2}{3 h^{2} s^{\prime}}\right) \cdot T^{3}\right] \\
& a_{16}=\frac{1}{s^{\prime}}\left[-\frac{1}{s^{\prime}} \frac{\partial^{2} h_{i}}{\partial \xi^{2}}\left(\frac{1}{R_{0}^{2}}-\frac{1}{R_{i}^{2}}\right) \frac{1}{2 h} \cdot T^{2}-\frac{\partial}{\partial \xi}\left(\frac{\partial h_{i}}{\partial \xi}\left(\frac{1}{R_{0}^{2}}+\frac{1}{R_{i}^{2}}\right) \frac{2}{3 h^{2} s^{\prime}}\right) \cdot T^{3}\right] \\
& a_{16}=-\frac{1}{s^{\prime}}\left[\frac{1}{s^{\prime}} \frac{\partial^{2} h_{i}}{\partial \xi^{2}}\left(\frac{1}{R_{0}^{2}}-\frac{1}{R_{i}^{2}}\right) \frac{1}{2 h} \frac{h^{2}}{4} \zeta^{2}+\frac{1}{s^{\prime}} \frac{\partial^{2} h_{i}}{\partial \xi^{2}}\left(\frac{1}{R_{0}^{2}}+\frac{1}{R_{i}^{2}}\right) \frac{2}{3 h^{2}} \frac{h^{3}}{8} \zeta^{3}\right] \\
& a_{16}=-\frac{1}{s^{\prime}} \frac{\partial^{2} h_{i}}{\partial \xi^{2}}\left[\left(\frac{1}{R_{0}^{2}}-\frac{1}{R_{i}^{2}}\right) \frac{h \zeta^{2}}{8}+\left(\frac{1}{R_{0}^{2}}+\frac{1}{R_{i}^{2}}\right) \frac{h \zeta^{3}}{12}\right]
\end{aligned}
$$

Portanto:

$$
a_{16}=-\frac{1}{s^{\prime}} \frac{\partial^{2} h_{i}}{\partial \xi^{2}} \frac{\zeta^{2}}{4}\left[\left(\frac{1}{R_{0}^{2}}-\frac{1}{R_{i}^{2}}\right) \frac{1}{2}+\left(\frac{1}{R_{0}^{2}}+\frac{1}{R_{i}^{2}}\right) \frac{\zeta}{3}\right]
$$

- Isolar $W_{4}^{i}$

$$
\begin{aligned}
& a_{17}= \frac{1}{s^{\prime}}\left[\frac{\partial}{\partial \xi}\left(-\left(\frac{1}{s^{\prime}} \frac{\partial}{\partial \xi}\left(\left.h_{i} \cdot R^{3}\right|_{\zeta=+1}\right)-\frac{1}{s^{\prime}} \frac{\partial}{\partial \xi}\left(\left.h_{i} \cdot R^{3}\right|_{\zeta=-1}\right)\right) \frac{1}{2 h}\right) \cdot T^{2}\right. \\
&\left.+\frac{\partial}{\partial \xi}\left(-\left(\frac{\partial}{\partial \xi}\left(\left.h_{i} \cdot R^{3}\right|_{\zeta=+1}\right)+\frac{\partial}{\partial \xi}\left(\left.h_{i} \cdot R^{3}\right|_{\zeta=-1}\right)\right) \frac{2}{3 h^{2} s^{\prime}}\right) \cdot T^{3}\right] \\
& a_{17}= \frac{1}{s^{\prime}}\left[-\frac{1}{s^{\prime}} \frac{\partial^{2} h_{i}}{\partial \xi^{2}}\left(R_{0}^{3}-R_{i}^{3}\right) \frac{1}{2 h} \cdot T^{2}-\frac{\partial^{2} h_{i}}{\partial \xi^{2}}\left(R_{0}^{3}+R_{i}^{3}\right) \frac{2}{3 h^{2} s^{\prime}} \cdot T^{3}\right] \\
& a_{17}=-\frac{1}{s^{\prime 2}} \frac{\partial^{2} h_{i}}{\partial \xi^{2}}\left[\left(R_{0}^{3}-R_{i}^{3}\right) \frac{1}{2 h} \frac{h^{2}}{4} \zeta^{2}+\left(R_{0}^{3}+R_{i}^{3}\right) \frac{2}{3 h^{2}} \frac{h^{3}}{8} \zeta^{3}\right]
\end{aligned}
$$

Portanto: 


$$
a_{17}=-\frac{1}{s^{\prime 2}} \frac{\partial^{2} h_{i}}{\partial \xi^{2}} \frac{\zeta^{2}}{4} h\left[\left(R_{0}^{3}-R_{i}^{3}\right) \frac{1}{2}+\left(R_{0}^{3}+R_{i}^{3}\right) \frac{\zeta}{3}\right]
$$

\subsection{2}

Deformação Circunferencial, $\epsilon_{\theta \theta}$

$$
\epsilon_{\theta \theta}=\frac{W(\xi, \zeta)}{R(\xi, \zeta)}
$$

Onde:

$$
\begin{gathered}
W(\xi, \zeta)=W_{0}(\xi)+\frac{W_{1}(\xi)}{R(\xi, \zeta)}+W_{2}(\xi) \cdot R(\xi, \zeta)+\frac{W_{3}(\xi)}{R(\xi, \zeta)^{2}}+W_{4}(\xi) \cdot R(\xi, \zeta)^{3} \\
\epsilon_{\theta \theta}=\frac{W_{0}(\xi)+\frac{W_{1}(\xi)}{R(\xi, \zeta)}+W_{2}(\xi) \cdot(R(\xi, \zeta))+\frac{W_{3}(\xi)}{(R(\xi, \zeta))^{2}}+W_{4}(\xi) \cdot(R(\xi, \zeta))^{3}}{R(\xi, \zeta)}
\end{gathered}
$$

Onde:

$$
\begin{gathered}
R(\xi, \zeta)=R_{2}+T \\
\epsilon_{\theta \theta}=\frac{W_{0}(\xi)+\frac{W_{1}(\xi)}{R_{2}+T}+W_{2}(\xi) \cdot\left(R_{2}+T\right)+\frac{W_{3}(\xi)}{\left(R_{2}+T\right)^{2}}+W_{4}(\xi) \cdot\left(R_{2}+T\right)^{3}}{R_{2}+T} \\
\epsilon_{\theta \theta}=\frac{\sum_{i=1}^{k} h_{i} \cdot W_{0}^{i}}{R_{2}+T}+\frac{\sum_{i=1}^{k} h_{i} \cdot W_{1}^{i}}{\left(R_{2}+T\right)^{2}} \\
+\sum_{i=1}^{k} h_{i} \cdot W_{2}^{i}+\frac{\sum_{i=1}^{k} h_{i} \cdot W_{3}^{i}}{\left(R_{2}+T\right)^{3}}+\sum_{i=1}^{k} h_{i} \cdot W_{4}^{i} \cdot(R 2+T)^{2}
\end{gathered}
$$

- Isolar $U_{1}^{i}$

$$
a_{21}=0
$$

- Isolar $\gamma_{1}^{i}$

$$
a_{22}=0
$$

- Isolar $W_{0}^{i}$

$$
\begin{aligned}
a_{23} & =\frac{h_{i}}{R_{2}+T} \\
a_{23} & =\frac{h_{i}}{R_{2}+\frac{h}{2} \zeta}
\end{aligned}
$$


- Isolar $W_{1}^{i}$

$$
\begin{gathered}
a_{24}=\frac{h_{i}}{\left(R_{2}+T\right)^{2}} \\
a_{24}=\frac{h_{i}}{\left(R_{2}+\frac{h}{2} \zeta\right)^{2}}
\end{gathered}
$$

- Isolar $W_{2}^{i}$

$$
a_{25}=h_{i}
$$

- Isolar $W_{3}^{i}$

$$
\begin{aligned}
& a_{26}=\frac{h_{i}}{\left(R_{2}+T\right)^{3}} \\
& a_{26}=\frac{h_{i}}{\left(R_{2}+\frac{h}{2} \zeta\right)^{3}}
\end{aligned}
$$

- Isolar $W_{3}^{i}$

$$
\begin{gathered}
a_{27}=h_{i} \cdot\left(R_{2}+T\right)^{2} \\
a_{27}=h_{i} \cdot\left(R_{2}+\frac{h}{2} \zeta\right)^{2}
\end{gathered}
$$

\subsection{3}

Deformação Radial, $\epsilon_{\zeta \zeta}$

$$
\epsilon_{\zeta \zeta}=\frac{\partial W}{\partial T}
$$

Onde:

$$
\begin{gathered}
W(\xi, \zeta)=W_{0}(\xi)+\frac{W_{1}(\xi)}{R(\xi, \zeta)}+W_{2}(\xi) \cdot R(\xi, \zeta)+\frac{W_{3}(\xi)}{R(\xi, \zeta)^{2}}+W_{4}(\xi) \cdot R(\xi, \zeta)^{3} \\
\epsilon_{\zeta \zeta}=\frac{\partial}{\partial T}\left[W_{0}(\xi)+\frac{W_{1}(\xi)}{R(\xi, \zeta)}+W_{2}(\xi) \cdot R(\xi, \zeta)+\frac{W_{3}(\xi)}{R(\xi, \zeta)^{2}}+W_{4}(\xi) \cdot R(\xi, \zeta)^{3}\right] \\
\text { Onde: } R(\xi, \zeta)=R_{2}+T \\
\epsilon_{\zeta \zeta}=\frac{\partial}{\partial T}\left[W_{0}(\xi)+\frac{W_{1}(\xi)}{R_{2}+T}+W_{2}(\xi) \cdot\left(R_{2}+T\right)+\frac{W_{3}(\xi)}{\left(R_{2}+T\right)^{2}}+W_{4}(\xi) \cdot\left(R_{2}+T\right)^{3}\right] \\
\epsilon_{\zeta \zeta}=0+\frac{\partial}{\partial T}\left(W_{1} \cdot\left(R_{2}+T\right)^{-1}\right)+W_{2}+\frac{\partial}{\partial T}\left(W_{3} \cdot\left(R_{2}+T\right)^{-2}\right)+\frac{\partial}{\partial T}\left(W_{4} \cdot\left(R_{2}+T\right)^{3}\right)
\end{gathered}
$$




$$
\begin{gathered}
\epsilon_{\zeta \zeta}=-W_{1}\left(R_{2}+T\right)^{-2}+W_{2}-2 W_{3}\left(R_{2}+T\right)^{-3}+3 W_{4}\left(R_{2}+T\right)^{2} \\
\epsilon_{\zeta \zeta}=-\frac{\sum_{i=1}^{k} h_{i} W_{1}^{i}}{\left(R_{2}+T\right)^{2}}+\sum_{i=1}^{k} h_{i} W_{2}^{i}-2 \frac{\sum_{i=1}^{k} h_{i} W_{3}^{i}}{\left(R_{2}+T\right)^{3}}+3\left(R_{2}+T\right)^{2} \sum_{i=1}^{k} h_{i} W_{4}^{i}
\end{gathered}
$$

- Isolar $U_{1}^{i}$

$$
a_{31}=0
$$

- Isolar $\gamma_{1}^{i}$

$$
a_{32}=0
$$

- Isolar $W_{0}^{i}$

$$
a_{33}=0
$$

- Isolar $W_{1}^{i}$

$$
\begin{aligned}
a_{34} & =-\frac{h_{i}}{\left(R_{2}+T\right)^{2}} \\
a_{34} & =-\frac{h_{i}}{\left(R_{2}+\frac{h}{2} \zeta\right)^{2}}
\end{aligned}
$$

- Isolar $W_{2}^{i}$

$$
a_{35}=h_{i}
$$

- Isolar $W_{3}^{i}$

$$
\begin{gathered}
a_{36}=-\frac{2 h_{i}}{\left(R_{2}+T\right)^{3}} \\
a_{36}=-\frac{2 h_{i}}{\left(R_{2}+\frac{h}{2} \zeta\right)^{3}}
\end{gathered}
$$

- Isolar $W_{4}^{i}$

$$
\begin{gathered}
a_{37}=3 h_{i}\left(R_{2}+T\right)^{2} \\
a_{37}=3 h_{i}\left(R_{2}+\frac{h}{2} \zeta\right)^{2}
\end{gathered}
$$

\subsection{4}

Deformação , $\gamma_{\xi \zeta}$

$$
\gamma_{\xi \zeta}=\frac{1}{s^{\prime}} \frac{\partial W}{\partial \xi}+\frac{\partial U_{1}}{\partial T}
$$




$$
\begin{array}{r}
\gamma_{\xi \zeta}=\frac{1}{s^{\prime}} \frac{\partial}{\partial \xi}\left(W_{0}(\xi)+\frac{W_{1}(\xi)}{R(\xi, \zeta)}+W_{2}(\xi) \cdot R(\xi, \zeta)+\frac{W_{3}(\xi)}{R(\xi, \zeta)^{2}}+W_{4}(\xi) \cdot R(\xi, \zeta)^{3}\right) \\
+\frac{\partial}{\partial T}\left(U_{1}^{0}(\xi)+\gamma_{1}(\xi) \cdot T+\phi_{1}(\xi) \cdot T^{2}+\psi_{1}(\xi) \cdot T^{3}\right) \\
\gamma_{\xi \zeta}=\frac{1}{s^{\prime}} \frac{\partial}{\partial \xi}\left[\sum_{i=1}^{k} h_{i} W_{0}^{i}+\frac{1}{R} \cdot \sum_{i=1}^{k} h_{i} W_{1}^{i}+R \cdot \sum_{i=1}^{k} h_{i} W_{2}^{i}+\frac{1}{R^{2}} \cdot \sum_{i=1}^{k} h_{i} W_{3}^{i}\right. \\
\left.+R^{3} \cdot \sum_{i=1}^{k} h_{i} W_{4}^{i}\right]+\frac{\partial}{\partial T}\left[\sum_{i=1}^{k} h_{i} U_{0}^{i}+T \cdot \sum_{i=1}^{k} h_{i} \gamma_{0}^{i}+\phi_{1}(\xi) \cdot T^{2}+\psi_{1}(\xi) \cdot T^{3}\right]
\end{array}
$$

Pela Condição de Contorno Naturais, equções e , temos:

$$
\begin{gathered}
\phi_{1}(\xi)=-\left(\left.\frac{1}{s^{\prime}} \frac{\partial W}{\partial \xi}\right|_{\zeta=+1}-\left.\frac{1}{s^{\prime}} \frac{\partial W}{\partial \xi}\right|_{\zeta=-1}\right) \cdot \frac{1}{2 h} \\
\psi_{1}(\xi)=-\left(\left.\frac{1}{s^{\prime}} \frac{\partial W}{\partial \xi}\right|_{\zeta=+1}+\left.\frac{1}{s^{\prime}} \frac{\partial W}{\partial \xi}\right|_{\zeta=-1}\right) \cdot \frac{2}{3 h^{2} s^{\prime}}-\frac{4 \gamma_{1}}{3 h^{2}}
\end{gathered}
$$

Substituindo:

$$
\begin{gathered}
\gamma_{\xi \zeta}=\frac{1}{s^{\prime}} \frac{\partial}{\partial \xi}\left[\sum_{i=1}^{k} h_{i} W_{0}^{i}+\frac{1}{R(\xi, \zeta)} \cdot \sum_{i=1}^{k} h_{i} W_{1}^{i}+R(\xi, \zeta) \cdot \sum_{i=1}^{k} h_{i} W_{2}^{i}+\frac{1}{R(\xi, \zeta)^{2}} \cdot \sum_{i=1}^{k} h_{i} W_{3}^{i}\right. \\
\left.+R(\xi, \zeta)^{3} \cdot \sum_{i=1}^{k} h_{i} W_{4}^{i}\right]+\frac{\partial}{\partial T}\left[\sum_{i=1}^{k} h_{i} U_{0}^{i}+T \cdot \sum_{i=1}^{k} h_{i} \gamma_{0}^{i}-\left(\left.\frac{1}{s^{\prime}} \frac{\partial W}{\partial \xi}\right|_{\zeta=+1}-\left.\frac{1}{s^{\prime}} \frac{\partial W}{\partial \xi}\right|_{\zeta=-1}\right) \cdot \frac{1}{2 h} \cdot T^{2}\right. \\
\left.+\left(-\left(\left.\frac{1}{s^{\prime}} \frac{\partial W}{\partial \xi}\right|_{\zeta=+1}+\left.\frac{1}{s^{\prime}} \frac{\partial W}{\partial \xi}\right|_{\zeta=-1}\right) \cdot \frac{2}{3 h^{2} s^{\prime}}-\frac{4 \gamma_{1}}{3 h^{2}}\right) \cdot T^{3}\right] \\
\gamma_{\xi \zeta}=\frac{1}{s^{\prime}} \frac{\partial}{\partial \xi}\left[\sum_{i=1}^{k} h_{i} W_{0}^{i}+\frac{1}{(R 2+T)} \cdot \sum_{i=1}^{k} h_{i} W_{1}^{i}+(R 2+T) \cdot \sum_{i=1}^{k} h_{i} W_{2}^{i}+\frac{1}{(R 2+T)^{2}} \cdot \sum_{i=1}^{k} h_{i} W_{3}^{i}\right. \\
\left.+(R 2+T)^{3} \cdot \sum_{i=1}^{k} h_{i} W_{4}^{i}\right]+\frac{\partial}{\partial T}\left[\sum_{i=1}^{k} h_{i} U_{0}^{i}+T \cdot \sum_{i=1}^{k} h_{i} \gamma_{0}^{i}-\left(\left.\frac{1}{s^{\prime}} \frac{\partial W}{\partial \xi}\right|_{\zeta=+1}-\left.\frac{1}{s^{\prime}} \frac{\partial W}{\partial \xi}\right|_{\zeta=-1}\right) \cdot \frac{1}{2 h} \cdot T^{2}\right. \\
+\left(-\left(\left.\frac{1}{s^{\prime}} \frac{\partial W}{\partial \xi}\right|_{\zeta=+1}+\left.\frac{1}{s^{\prime}} \frac{\partial W}{\partial \xi}\right|_{\zeta=-1}\right) \cdot \frac{2}{3 h^{2} s^{\prime}}-\frac{4}{3 h^{2}} \cdot \sum_{i=1}^{k} \frac{\left.\left.h_{i} \gamma_{1}^{i}\right) \cdot T^{3}\right]}{2}\right]
\end{gathered}
$$

- Isolar $U_{0}^{i}$

$$
a_{41}=0
$$


- Isolar $\gamma_{1}^{i}$

$$
\begin{gathered}
a_{42}=h_{i}-\frac{\partial}{\partial T}\left(\frac{4}{3 h^{2}} h_{i} T^{3}\right) \\
a_{42}=h_{i}-\frac{4}{3 h^{2}} h_{i} 3 T^{2}
\end{gathered}
$$

Substituindo T pela equação :

$$
a_{42}=h_{i}-\frac{4}{3 h^{2}} h_{i} 3 \frac{h^{2}}{4} \zeta^{2}
$$

Portanto:

$$
a_{42}=h_{i}\left(1-\zeta^{2}\right)
$$

- Isolar $W_{0}^{i}$

$$
\begin{gathered}
a_{43}=\frac{1}{s^{\prime}} \frac{\partial h_{i}}{\partial \xi}+\frac{\partial}{\partial T}\left[\left(\frac{1}{s^{\prime}} \frac{\partial h_{i}}{\partial \xi}-\frac{1}{s^{\prime}} \frac{\partial h_{i}}{\partial \xi}\right) \frac{1}{2 h} \cdot T^{2}\right]+\frac{\partial}{\partial T}\left[\left(-\left(\frac{\partial h_{i}}{\partial \xi}+\frac{\partial h_{i}}{\partial \xi}\right) \frac{2}{3 h^{2} s^{\prime}}\right) \cdot T^{3}\right] \\
a_{43}=\frac{1}{s^{\prime}} \frac{\partial h_{i}}{\partial \xi}+\frac{\partial}{\partial T}\left[0 \cdot \frac{1}{2 h} \cdot T^{2}\right]+\frac{\partial}{\partial T}\left[\left(-\left(2 \frac{\partial h_{i}}{\partial \xi}\right) \frac{2}{3 h^{2} s^{\prime}}\right) \cdot T^{3}\right] \\
a_{43}=\frac{1}{s^{\prime}} \frac{\partial h_{i}}{\partial \xi}-\frac{4}{3 h^{2} s^{\prime}} \frac{\partial h_{i}}{\partial \xi} \cdot 3 T^{2} \\
a_{43}=\frac{1}{s^{\prime}} \frac{\partial h_{i}}{\partial \xi}-\frac{1}{s^{\prime}} \frac{\partial h_{i}}{\partial \xi} \frac{4}{3 h^{2} s^{\prime}} \cdot 3 T^{2}
\end{gathered}
$$

Substituindo T pela equação :

$$
\begin{gathered}
a_{43}=\frac{1}{s^{\prime}} \frac{\partial h_{i}}{\partial \xi}-\frac{1}{s^{\prime}} \frac{\partial h_{i}}{\partial \xi} \frac{4}{3 h^{2} s^{\prime}} \cdot 3 \frac{h^{2}}{4} \zeta^{2} \\
a_{43}=\frac{1}{s^{\prime}} \frac{\partial h_{i}}{\partial \xi}-\frac{1}{s^{\prime}} \frac{\partial h_{i}}{\partial \xi} \zeta^{2}
\end{gathered}
$$

Portanto:

$$
a_{43}=\frac{1}{s^{\prime}} \frac{\partial h_{i}}{\partial \xi}\left(1-\zeta^{2}\right)
$$

- Isolar $W_{1}^{i}$

$$
\begin{gathered}
a_{44}=\frac{1}{s^{\prime}} \frac{\partial}{\partial \xi}\left(\frac{h_{i}}{R(\xi, \zeta)}\right)-\frac{\partial}{\partial T}\left[\left(\frac{1}{s^{\prime}} \frac{\partial}{\partial \xi}\left(\frac{h_{i}}{\left.R\right|_{\zeta=+1}}\right)-\frac{1}{s^{\prime}} \frac{\partial}{\partial \xi}\left(\frac{h_{i}}{\left.R\right|_{\zeta=+1}}\right)\right) \frac{1}{2 h} \cdot T^{2}\right] \\
+\frac{\partial}{\partial T}\left[-\left(\frac{\partial}{\partial \xi}\left(\frac{h_{i}}{\left.R\right|_{\zeta=-1}}\right)-\frac{\partial}{\partial \xi}\left(\frac{h_{i}}{\left.R\right|_{\zeta=-1}}\right)\right) \frac{2}{3 h^{2} s^{\prime}} \cdot T^{3}\right]
\end{gathered}
$$




$$
\begin{array}{r}
a_{44}=\frac{1}{s^{\prime}} \frac{1}{R(\zeta)} \frac{\partial h_{i}}{\partial \xi}-\frac{\partial}{\partial T}\left[\frac{1}{s^{\prime}} \frac{\partial h_{i}}{\partial \xi}\left(\frac{1}{R_{0}}-\frac{1}{R_{i}}\right) \frac{1}{2 h} \cdot T^{2}\right] \\
+\frac{\partial}{\partial T}\left[-\frac{\partial h_{i}}{\partial \xi}\left(\frac{1}{R_{0}}+\frac{1}{R_{i}}\right) \frac{2}{3 h^{2} s^{\prime}} \cdot T^{3}\right] \\
a_{44}=\frac{1}{s^{\prime}} \frac{1}{R_{2}+T} \frac{\partial h_{i}}{\partial \xi}-\frac{1}{s^{\prime}} \frac{\partial h_{i}}{\partial T}\left(\frac{1}{R_{0}}-\frac{1}{R_{i}}\right) \frac{1}{2 h} \frac{\partial}{\partial T}\left(T^{2}\right) \\
\quad-\frac{\partial h_{i}}{\partial \xi}\left(\frac{1}{R_{0}}+\frac{1}{R_{i}}\right) \frac{2}{3 h^{2} s^{\prime}} \frac{\partial}{\partial T}\left(T^{3}\right) \\
a_{44}=\frac{1}{s^{\prime}} \frac{1}{R_{2}+T} \frac{\partial h_{i}}{\partial \xi}-\frac{1}{s^{\prime}} \frac{\partial h_{i}}{\partial T}\left(\frac{1}{R_{0}}-\frac{1}{R_{i}}\right) \frac{1}{2 h} \cdot 2 T \\
-\frac{\partial h_{i}}{\partial \xi}\left(\frac{1}{R_{0}}+\frac{1}{R_{i}}\right) \frac{2}{3 h^{2} s^{\prime}} \cdot 3 T^{2}
\end{array}
$$

Substituindo T, pela equação :

$$
\begin{aligned}
a_{44}=\frac{1}{s^{\prime}} \frac{1}{R_{2}+\frac{h}{2} \zeta} \frac{\partial h_{i}}{\partial \xi}-\frac{1}{s^{\prime}} \frac{\partial h_{i}}{\partial T}\left(\frac{1}{R_{0}}-\frac{1}{R_{i}}\right) \frac{1}{2 h} \cdot 2 \frac{h}{2} \zeta \\
-\frac{\partial h_{i}}{\partial \xi}\left(\frac{1}{R_{0}}+\frac{1}{R_{i}}\right) \frac{2}{3 h^{2} s^{\prime}} \cdot 3 T^{2}
\end{aligned}
$$

Portanto:

$$
a_{44}=\frac{1}{s^{\prime}} \frac{1}{R_{2}+\frac{h}{2} \zeta} \frac{\partial h_{i}}{\partial \xi}\left[\frac{1}{R}-\left(\frac{1}{R_{0}}-\frac{1}{R_{i}}\right) \frac{\zeta}{2}-\left(\frac{1}{R_{0}}+\frac{1}{R_{i}}\right) \frac{\zeta^{2}}{2}\right]
$$

- Isolar $W_{2}^{i}$

$$
\begin{array}{r}
a_{45}=\frac{1}{s^{\prime}} \frac{\partial}{\partial \xi}\left(R(\xi, \zeta) \cdot h_{i}\right)-\frac{\partial}{\partial T}\left[\left(\frac{1}{s^{\prime}} \frac{\partial}{\partial \xi}\left(\left.h_{i} \cdot R\right|_{\zeta=+1}\right)-\frac{1}{s^{\prime}} \frac{\partial}{\partial \xi}\left(\left.h_{i} \cdot R\right|_{\zeta=+1}\right)\right) \frac{1}{2 h} \cdot T^{2}\right] \\
+\frac{\partial}{\partial T}\left[-\left(\frac{\partial}{\partial \xi}\left(\left.h_{i} \cdot R\right|_{\zeta=-1}\right)-\frac{\partial}{\partial \xi}\left(\left.h_{i} \cdot R\right|_{\zeta=-1}\right)\right) \frac{2}{3 h^{2} s^{\prime}} \cdot T^{3}\right] \\
a_{45}=\frac{1}{s^{\prime}} \frac{\partial h_{i}}{\partial \xi} R(\zeta)-\frac{\partial}{\partial T}\left[\frac{1}{s^{\prime}} \frac{\partial h_{i}}{\partial \xi}\left(R_{0}-R_{i}\right) \frac{1}{2 h} \cdot T^{2}\right] \\
+\frac{\partial}{\partial T}\left[-\frac{\partial h_{i}}{\partial \xi}\left(R_{0}+R_{i}\right) \frac{2}{3 h^{2} s^{\prime}} \cdot T^{3}\right]
\end{array}
$$




$$
\begin{aligned}
& a_{45}=\frac{1}{s^{\prime}} \frac{\partial h_{i}}{\partial \xi}\left(R_{2}+T\right)-\frac{1}{s^{\prime}} \frac{\partial h_{i}}{\partial \xi}\left(R_{0}-R_{i}\right) \frac{1}{2 h} \cdot \frac{\partial}{\partial T}\left(T^{2}\right)-\frac{\partial h_{i}}{\partial \xi}\left(R_{0}+R_{i}\right) \frac{2}{3 h^{2} s^{\prime}} \cdot \frac{\partial}{\partial T}\left(T^{3}\right) \\
& a_{45}=\frac{1}{s^{\prime}} \frac{\partial h_{i}}{\partial \xi}\left(R_{2}+T\right)-\frac{1}{s^{\prime}} \frac{\partial h_{i}}{\partial \xi}\left(R_{0}-R_{i}\right) \frac{1}{2 h} \cdot 2 T-\frac{\partial h_{i}}{\partial \xi}\left(R_{0}+R_{i}\right) \frac{2}{3 h^{2} s^{\prime}} \cdot 3 T^{2}
\end{aligned}
$$

Substituindo T, pela equção :

$$
\begin{gathered}
a_{45}=\frac{1}{s^{\prime}} \frac{\partial h_{i}}{\partial \xi}\left(R_{2}+\frac{h}{2} \zeta\right)-\frac{1}{s^{\prime}} \frac{\partial h_{i}}{\partial \xi}\left(R_{0}-R_{i}\right) \frac{1}{2 h} \cdot 2 \frac{h}{2} \zeta-\frac{1}{s^{\prime}} \frac{\partial h_{i}}{\partial \xi}\left(R_{0}+R_{i}\right) \frac{2}{3 h^{2}} \cdot 3 \frac{h^{2}}{4} \zeta^{2} \\
a_{45}=\frac{1}{s^{\prime}} \frac{\partial h_{i}}{\partial \xi}\left(\left(R_{2}+\frac{h}{2} \zeta\right)-\left(R_{0}-R_{i}\right) \frac{\zeta}{2}-\left(R_{0}+R_{i}\right) \frac{\zeta^{2}}{2}\right)
\end{gathered}
$$

- Isolar $W_{3}^{i}$

$$
\begin{aligned}
& a_{46}=\frac{1}{s^{\prime}} \frac{\partial}{\partial \xi}\left(\frac{h_{i}}{R(\xi, \zeta)^{2}}\right)-\frac{\partial}{\partial T}\left[\left(\frac{1}{s^{\prime}} \frac{\partial}{\partial \xi}\left(\frac{h_{i}}{\left.R^{2}\right|_{\zeta=+1}}\right)-\frac{1}{s^{\prime}} \frac{\partial}{\partial \xi}\left(\frac{h_{i}}{\left.R^{2}\right|_{\zeta=+1}}\right)\right) \frac{1}{2 h} \cdot T^{2}\right] \\
& +\frac{\partial}{\partial T}\left[-\left(\frac{\partial}{\partial \xi}\left(\frac{h_{i}}{\left.R^{2}\right|_{\zeta=-1}}\right)-\frac{\partial}{\partial \xi}\left(\frac{h_{i}}{\left.R^{2}\right|_{\zeta=-1}}\right)\right) \frac{2}{3 h^{2} s^{\prime}} \cdot T^{3}\right] \\
& a_{46}=\frac{1}{s^{\prime}} \frac{\partial h_{i}}{\partial \xi} \frac{1}{R^{2}}-\frac{\partial}{\partial T}\left[\left(\frac{1}{s^{\prime}} \frac{\partial h_{i}}{\partial \xi}\left(\frac{1}{R_{0}^{2}}-\frac{1}{R_{i}^{2}}\right) \frac{1}{2 h} \cdot T^{2}\right]\right. \\
& -\frac{\partial}{\partial T}\left[\frac{\partial h_{i}}{\partial \xi}\left(\frac{1}{R_{0}^{2}}+\frac{1}{R_{i}^{2}}\right) \frac{2}{3 h^{2} s^{\prime}} \cdot T^{3}\right] \\
& a_{46}=\frac{1}{s^{\prime}} \frac{\partial h_{i}}{\partial \xi} \frac{1}{\left(R_{2}+T\right)^{2}}-\frac{1}{s^{\prime}} \frac{\partial h_{i}}{\partial \xi}\left(\frac{1}{R_{0}^{2}}-\frac{1}{R_{i}^{2}}\right) \frac{1}{2 h} \cdot \frac{\partial}{\partial T}\left(T^{2}\right) \\
& -\frac{1}{s^{\prime}} \frac{\partial h_{i}}{\partial \xi}\left(\frac{1}{R_{0}^{2}}+\frac{1}{R_{i}^{2}}\right) \frac{2}{3 h^{2}} \cdot \frac{\partial}{\partial T}\left(T^{3}\right) \\
& a_{46}=\frac{1}{s^{\prime}} \frac{\partial h_{i}}{\partial \xi} \frac{1}{\left(R_{2}+T\right)^{2}}-\frac{1}{s^{\prime}} \frac{\partial h_{i}}{\partial \xi}\left(\frac{1}{R_{0}^{2}}-\frac{1}{R_{i}^{2}}\right) \frac{1}{2 h} \cdot 2 T \\
& -\frac{1}{s^{\prime}} \frac{\partial h_{i}}{\partial \xi}\left(\frac{1}{R_{0}^{2}}+\frac{1}{R_{i}^{2}}\right) \frac{2}{3 h^{2}} \cdot 3 T^{2}
\end{aligned}
$$


Substituindo T pela equação :

$$
\begin{aligned}
a_{46}=\frac{1}{s^{\prime}} \frac{\partial h_{i}}{\partial \xi} \frac{1}{\left(R_{2}+\frac{h}{2} \zeta\right)^{2}}-\frac{1}{s^{\prime}} \frac{\partial h_{i}}{\partial \xi}\left(\frac{1}{R_{0}^{2}}-\frac{1}{R_{i}^{2}}\right) \frac{1}{2 h} \cdot 2 \frac{h}{2} \zeta \\
-\frac{1}{s^{\prime}} \frac{\partial h_{i}}{\partial \xi}\left(\frac{1}{R_{0}^{2}}+\frac{1}{R_{i}^{2}}\right) \frac{2}{3 h^{2}} \cdot 3 \frac{h^{2}}{4} \zeta^{2} \\
a_{46}=\frac{1}{s^{\prime}} \frac{\partial h_{i}}{\partial \xi}\left(\frac{1}{\left(R_{2}+\frac{h}{2} \zeta\right)^{2}}-\left(\frac{1}{R_{0}^{2}}-\frac{1}{R_{i}^{2}}\right) \frac{\zeta}{2}-\left(\frac{1}{R_{0}^{2}}+\frac{1}{R_{i}^{2}}\right) \frac{\zeta^{2}}{2}\right)
\end{aligned}
$$

- Isolar $W_{4}^{i}$

$$
\begin{aligned}
& a_{47}= \frac{1}{s^{\prime}} \frac{\partial}{\partial \xi}\left(R(\xi, \zeta)^{3} \cdot h_{i}\right)+\frac{\partial}{\partial T}\left[-\left(\frac{1}{s^{\prime}} \frac{\partial}{\partial \xi}\left(\left.h_{i} \cdot R^{3}\right|_{\zeta=+1}\right)-\frac{1}{s^{\prime}} \frac{\partial}{\partial \xi}\left(\left.h_{i} \cdot R^{3}\right|_{\zeta=+1}\right)\right) \frac{1}{2 h} \cdot T^{2}\right] \\
&+\frac{\partial}{\partial T}\left[-\left(\frac{\partial}{\partial \xi}\left(\left.h_{i} \cdot R^{3}\right|_{\zeta=-1}\right)-\frac{\partial}{\partial \xi}\left(\left.h_{i} \cdot R^{3}\right|_{\zeta=-1}\right)\right) \frac{2}{3 h^{2} s^{\prime}} \cdot T^{3}\right] \\
& a_{47}=\frac{1}{s^{\prime}} \frac{\partial h_{i}}{\partial \xi} R(\zeta)^{3}+\frac{\partial}{\partial T}\left[-\frac{1}{s^{\prime}} \frac{\partial h_{i}}{\partial \xi}\left(R_{0}^{3}-R_{i}^{3}\right) \frac{1}{2 h} \cdot T^{2}\right]-\frac{\partial}{\partial T}\left[\frac{1}{s^{\prime}} \frac{\partial h_{i}}{\partial \xi}\left(R_{0}^{3}+R_{i}^{3}\right) \frac{2}{3 h^{2}} \cdot T^{3}\right] \\
& a_{47}=\frac{1}{s^{\prime}} \frac{\partial h_{i}}{\partial \xi}\left(R_{2}+T\right)^{3}-\frac{1}{s^{\prime}} \frac{\partial h_{i}}{\partial \xi}\left(R_{0}^{3}-R_{i}^{3}\right) \frac{1}{2 h} \cdot \frac{\partial}{\partial T}\left(T^{2}\right) \\
& \quad-\frac{1}{s^{\prime}} \frac{\partial h_{i}}{\partial \xi}\left(R_{0}^{3}+R_{i}^{3}\right) \frac{2}{3 h^{2}} \cdot \frac{\partial}{\partial T}\left(T^{3}\right) \\
& a_{47}=\frac{1}{s^{\prime}} \frac{\partial h_{i}}{\partial \xi}\left(\left(R_{2}+T\right)^{3}-\left(R_{0}^{3}-R_{i}^{3}\right) \frac{1}{2 h} \cdot 2 T-\left(R_{0}^{3}+R_{i}^{3}\right) \frac{2}{3 h^{2}} \cdot 3 T^{2}\right) \\
& a_{47}=\frac{1}{s^{\prime}} \frac{\partial h_{i}}{\partial \xi}\left(R_{2}+T\right)^{3}-\frac{1}{s^{\prime}} \frac{\partial h_{i}}{\partial \xi}\left(R_{0}^{3}-R_{i}^{3}\right) \frac{1}{2 h} \cdot 2 T \\
&\left.\frac{\partial h_{0}}{\partial \xi}+R_{i}^{3}\right) \frac{2}{3 h^{2}} \cdot 3 T^{2}
\end{aligned}
$$


Substituindo T pela equação :

$$
a_{47}=\frac{1}{s^{\prime}} \frac{\partial h_{i}}{\partial \xi}\left(\left(R_{2}+\frac{h}{2} \zeta\right)^{3}-\left(R_{0}^{3}-R_{i}^{3}\right) \frac{1}{2 h} \cdot 2 \frac{h}{2} \zeta-\left(R_{0}^{3}+R_{i}^{3}\right) \frac{2}{3 h^{2}} \cdot 3 \frac{h^{2}}{4} \zeta^{2}\right)
$$

Simplificando:

$$
a_{47}=\frac{1}{s^{\prime}} \frac{\partial h_{i}}{\partial \xi} \frac{1}{2}\left(\left(R_{2}+\frac{h}{2} \zeta\right)^{3}-\left(R_{0}^{3}-R_{i}^{3}\right) \cdot \zeta-\left(R_{0}^{3}+R_{i}^{3}\right) \cdot \zeta^{2}\right)
$$




\section{Referências Bibliográficas}

[1] ESPINOZA, H.G.S., Formulação de Cascas Espessas Axissimétricas Utilizando Elementos Finitos Enriquecidos, Dissertação de Mestrado, PUC-Rio, 2004.

[2] SOARES, J.C.V., Formulação Unificada Para a Análise de Cascas Cilíndricas Finas e Espessas pelo Método dos Elementos Finitos, Trabalho de Conclusão de Curso, 2015.

[3] ALMEIDA, C.A., Elementos Finitos na Engenharia Mecânica, Notas de Aula, 2016.

[4] DEN HARTOG, J.P., Advanced Strength of Materials, Dover

[5] DYM, C.L., Introduction to The Theory of Shells, Pergamon Press, 1974

[6] BATHE, K.J., Finite Element Procedures, Prentice Hall, 2006

[7] POPOV, E.P., Engineering Mechanics of Solids, Prentice Hall, 1998 